\title{
Entropic Fluctuations in Statistical Mechanics I. Classical Dynamical Systems
}

\author{
V. Jakšić1 ${ }^{1}$ C.-A. Pillet ${ }^{2}$, L. Rey-Bellet ${ }^{3}$ \\ ${ }^{1}$ Department of Mathematics and Statistics \\ McGill University \\ 805 Sherbrooke Street West \\ Montreal, QC, H3A 2K6, Canada \\ ${ }^{2}$ Centre de Physique Théorique* \\ Université du Sud Toulon-Var, B.P. 20132 \\ F-83957 La Garde Cedex, France \\ ${ }^{3}$ Department of Mathematics and Statistics \\ Lederle Graduate Research Tower, Box 34515 \\ University of Massachusetts \\ Amherst, MA 01003-4515, USA
}

October 31, 2018

\begin{abstract}
Within the abstract framework of dynamical system theory we describe a general approach to the Transient (or Evans-Searles) and Steady State (or Gallavotti-Cohen) Fluctuation Theorems of non-equilibrium statistical mechanics. Our main objective is to display the minimal, model independent mathematical structure at work behind fluctuation theorems. Besides its conceptual simplicity, another advantage of our approach is its natural extension to quantum statistical mechanics which will be presented in a companion paper. We shall discuss several examples including thermostated systems, open Hamiltonian systems, chaotic homeomorphisms of compact metric spaces and Anosov diffeomorphisms.
\end{abstract}

*UMR 6207: Université de Provence, Université de la Méditerranée, Université de Toulon et CNRS, FRUMAM 


\section{Contents}

1 Introduction

2 Basic notions

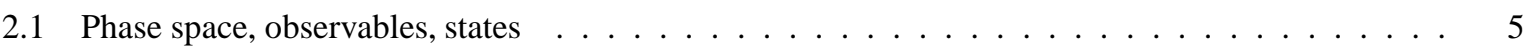

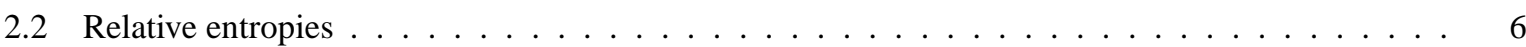

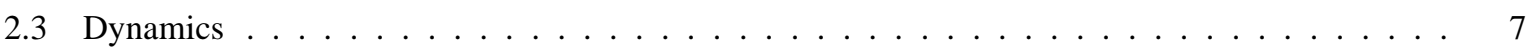

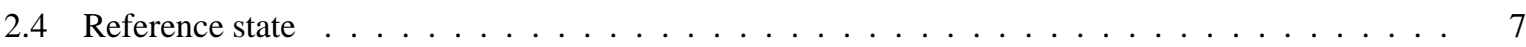

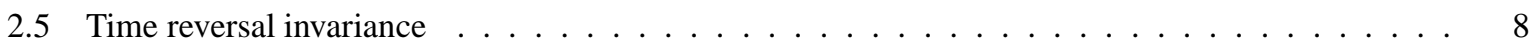

3 Finite time entropy production $\quad 8$

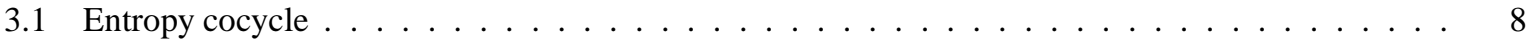

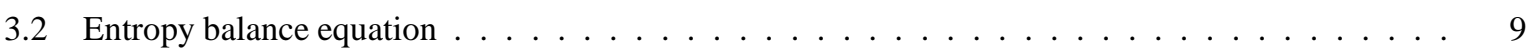

3.3 Finite time Evans-Searles symmetry $\ldots \ldots \ldots \ldots \ldots \ldots$

3.4 Entropy production observable $\ldots \ldots \ldots \ldots \ldots \ldots \ldots \ldots$

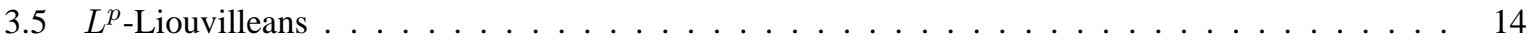

3.6 Examples: Differentiable dynamics and thermostated systems _ . . . . . . . . . . . . 15

4 Thermodynamics $\quad 17$

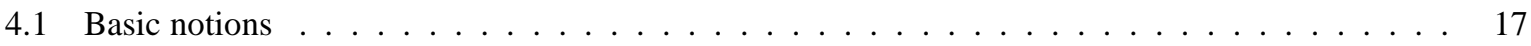

4.2 Finite time Generalized Evans-Searles symmetry $\ldots \ldots \ldots \ldots \ldots \ldots \ldots$

4.3 Finite time linear response theory . . . . . . . . . . . . . . . . . . . . . . . . 20

4.4 Example: Thermally driven open systems _ . . . . . . . . . . . . . . . . . . . . 21

5 The large time limit $\quad 24$

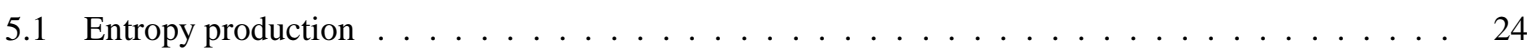

5.2 Linear response theory $\ldots \ldots \ldots \ldots \ldots \ldots \ldots$

5.3 The Evans-Searles Fluctuation Theorem . . . . . . . . . . . . . . . . . 29

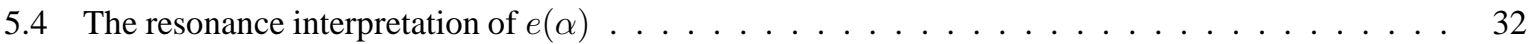

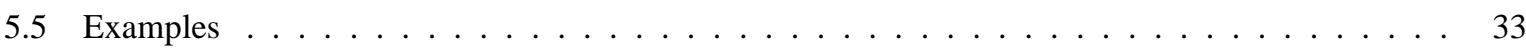

6 Non-equilibrium steady states

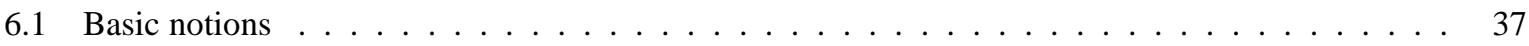

6.2 The Gallavotti-Cohen Fluctuation Theorem … . . . . . . . . . . . . . . . 38

6.3 The resonance interpretation of $e_{+}(\alpha) \ldots \ldots \ldots \ldots \ldots \ldots \ldots$

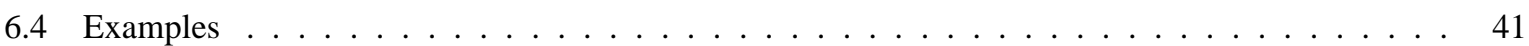


7 The principle of regular entropic fluctuations

8 Toy models

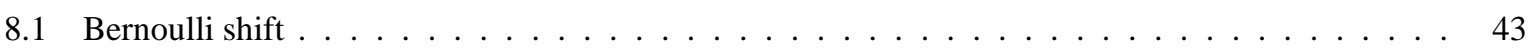

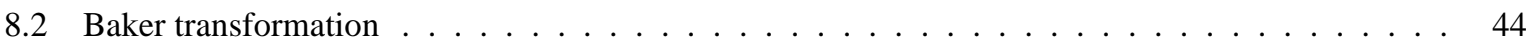

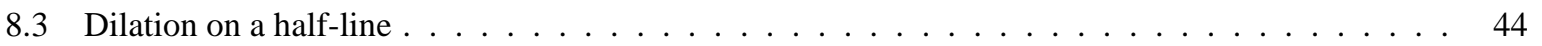

9 Gaussian dynamical systems 45

10 Homeomorphisms of compact metric spaces 49

10.1 Topological dynamics . . . . . . . . . . . . . . . . . . . . . . . 50

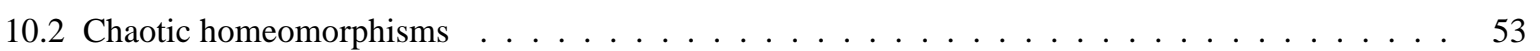

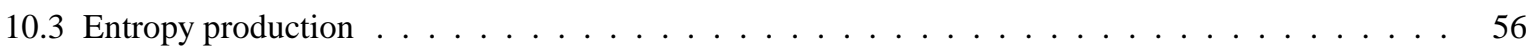

10.4 Reference measure and physical equivalence . . . . . . . . . . . . . . . . . 59

10.5 Markov chains . . . . . . . . . . . . . . . . . . . . . . . 61

11 Anosov diffeomorphisms $\quad 63$

Table of Abbreviations and Symbols

\section{Introduction}

This is the first in a series of papers devoted to the so-called Fluctuation Theorems of non-equilibrium statistical mechanics. This series is a part of the research program initiated in [Pi, JP1, JP2] that concerns the development of a mathematical theory of non-equilibrium statistical mechanics within the framework of dynamical systems.

The first fluctuation theorem in statistical mechanics goes back to 1905 and the celebrated work of Einstein on Brownian motion. The subsequent historical developments are reviewed in [RM] (see also the monographs [GM, $\underline{\mathrm{KTH}}]$ ) and we mention here only the classical results of Onsager [On1, On2], Green [Gr1, Gr2], and Kubo [Kub] which will be re-visited in this paper. Virtually all classical works on the subject concern the so called close to equilibrium regime in which the mechanical and thermodynamical forces (affinities) are weak. One of the key features of modern fluctuation theorems, suggested by numerical experiments [ECM] and established theoretically for the first time by Evans and Searles [ES] and by Gallavotti and Cohen [GC1, GC2], is that they hold for systems arbitrarily far from equilibrium and reduce to Green-Kubo formulas and Onsager relations in the linear regime near equilibrium. The seminal papers [ECM, ES, GC1, GC2] were followed by a vast body of theoretical, numerical and experimental works which are reviewed in [RM]. The Evans-Searles (ES) and Gallavotti-Cohen (GC) Fluctuation Theorems are the main topics of the present work.

The basic two paradigms for deterministic (dynamical system) non-equilibrium statistical mechanics are the so called thermostated systems and open systems. Thermostated systems are Hamiltonian systems (with finitely many degrees of freedom) driven out of equilibrium by an external (non-Hamiltonian) force and constrained by a deterministic thermostating force to stay on a surface of constant energy. Open systems are Hamiltonian systems consisting of a "small" Hamiltonian system (with finitely many degrees of freedom) interacting with, say two, 
"large" reservoirs which are infinitely extended Hamiltonian systems. The reservoirs are initially in thermal equilibrium at distinct temperatures and the temperature differential leads to a steady heat flux from the hotter to the colder reservoir across the small system. Throughout the main body of the paper we shall illustrate our results on an example of thermostated system and an example of open system.

The majority of works on fluctuation theorems concern classical physics. In the quantum case comparatively little is known and there are very few mathematically rigorous works on the subject (see [TM, DDM, Ro, Ku2]). The present paper, which concerns only the classical case, originates in our attempts to find a proper mathematical framework for the extensions of ES and GC Fluctuation Theorems to quantum physics. One of the difficulties in finding such a framework stems from the fact that it was already lacking at the classical level. Indeed, even the basic examples of thermostated systems and open systems were studied in the literature in an unrelated way and it was far from obvious which aspects of the theory are model dependent and which are universal. For example there was no clear universal rationale in the choice of the "entropy production" observable (also called "phase space contraction rate" or "dissipation function") which plays a central role in the theory.

A model independent definition of the entropy production has been proposed by Maes, in the context of stochastic (Markovian or Gibbsian) dynamical systems, see e.g. [Ma2]. We take here a different and complementary route and discuss non-equilibrium statistical mechanics within the context of deterministic dynamical systems. Our work is mostly of a review nature and we do not prove any new specific results. Rather we organize the existing set of ideas and results in an axiomatic abstract framework that unifies virtually all deterministic models discussed in the literature (in particular, open infinite systems and thermostated finite dimensional systems will be treated in a unified manner) and clarifies the mathematical structure of the theory. The framework has a direct extension to non-commutative dynamical systems and in particular to quantum mechanics and this will be the subject of the remaining papers in the series. Our principal new results concern the quantum case and we will focus here only on those aspects of the classical theory that can be extended, within the framework of dynamical systems, to quantum statistical mechanics.

We have made an attempt to expose the results in a pedagogical way and the only prerequisite for the principal part of the paper is a basic knowledge of probability and measure theory.

The paper is organized as follows.

In Section 2 we introduce our dynamical system setup and review the properties of relative entropies that we will need. In Section 3 we introduce the basic objects of the theory, the entropy cocycle and the entropy production observable, discuss their properties, and prove the finite time Evans-Searles Fluctuation Theorem. The results described in this section hold under minimal regularity assumptions that are satisfied in virtually all models of interest.

In Section 4 we start the discussion of thermodynamics by introducing control parameters (mechanical or thermodynamical forces) to our dynamical system setup. The finite time Evans-Searles Fluctuation Theorem is then generalized to this setting. Following the ideas of Gallavotti [Ga1] (see also [LS2]) we use this generalization to derive finite time Green-Kubo formulas and Onsager reciprocity relations.

The results of Sections 3 and 4 concern the system evolved over a finite interval of time and are very general. In particular, they do not require any ergodicity assumptions. Section 5 concerns the large time limit $t \rightarrow \infty$. Under suitable ergodicity assumptions we derive Evans-Searles Fluctuation Theorem on the basis of its universally valid finite time counterpart and prove the Green-Kubo formula and Onsager reciprocity relations.

Section 6 is devoted to the Gallavotti-Cohen Fluctuation Theorem. After introducing the key concept of nonequilibrium steady states (NESS), the GC Fluctuation Theorem is stated as, essentially, an ergodic-type hypothesis concerning the NESS and the entropy production observable. The Green-Kubo formula and Onsager reciprocity relations also follow from the GC Fluctuation Theorem.

One advantage of our abstract axiomatic framework is that it allows for a transparent comparison between the ES and GC Fluctuation Theorems. It turns out that from the mathematical point of view these two theorems are 
equivalent up to an exchange of limits (see Relation (7.62). This exchange of limits may fail even in some very simple models and its validity can be interpreted as an ergodic property of the underlying dynamical system. We raise this point to the Principle of Regular Entropic Fluctuations which is introduced and discussed in Section 7 After this work was completed we have learned that related ideas have been previously discussed in [RM].

Sections 8,11 are devoted to examples. In Section 8 we discuss several toy models which illustrate the optimality of our assumptions. In Section 9 we develop the non-equilibrium statistical mechanics of Gaussian dynamical systems. Chaotic homeomorphisms of compact metric spaces are discussed in Section 10. Finally, in Section 11 we discuss the non-equilibrium statistical mechanics of Anosov diffeomorphisms of compact manifolds. In each of these examples we verify the validity of the proposed principle of regular entropic fluctuations.

For the convenience of the reader, a table of frequently used abbreviations and symbols is provided on page 67.

The ergodic-type hypotheses introduced in this paper typically concern existence of certain limits as time $t \rightarrow \infty$, the regularity (differentiability, etc) properties of limiting functions w.r.t. control parameters, and the validity of exchange of order of limits and derivatives. The introduced hypotheses are minimal (i.e., sufficient and necessary) to derive fluctuation theorems and their implications from the universally valid structural theory discussed in Sections 2-4. The verification of these hypotheses in concrete models leads to a novel class of (analytically difficult) problems in ergodic theory of dynamical systems.

Acknowledgment. The research of V.J. was partly supported by NSERC. The research of C.-A.P. was partly supported by ANR (grant 09-BLAN-0098). The research of L.R.-B. was partly supported by NSF. We wish to thank C. Liverani and D. Ruelle for useful discussions. The results of this paper were presented by its authors in mini-courses at University of Cergy-Pontoise, Erwin Schrödinger Institute (Vienna), Centre de Physique Théorique (Marseille and Toulon), University of British Columbia (Vancouver), Ecole Polytechnique (Paris), Institut Henri Poincaré (Paris) and Ecole de Physique des Houches. The paper has gained a lot from these presentations and we wish to thank the respective institutions and F. Germinet, J. Yngvanson, R. Froese, S. Kuksin, G. Stoltz, J. Fröhlich for making these mini-courses possible.

\section{Basic notions}

\subsection{Phase space, observables, states}

Let $M$ be a set and $\mathcal{F}$ a $\sigma$-algebra in $M$. We shall refer to the measure space $(M, \mathcal{F})$ as the phase space. If $M$ is a topological space, we shall always take for $\mathcal{F}$ the Borel $\sigma$-algebra in $M$.

An observable is a measurable function $f: M \rightarrow \mathbb{C}$ and we denote by $\mathcal{O}(M)$ the complex vector space of all observables. $B(M)$ denotes the subspace of all bounded observables. Together with the norm $\|f\|=\sup _{x \in M}|f(x)|$, $B(M)$ is a Banach space. If $M$ is a topological space, $C(M)$ denotes the Banach space of all bounded continuous observables. The corresponding spaces of real valued observables are denoted by $\mathcal{O}_{\mathbb{R}}(M), B_{\mathbb{R}}(M), C_{\mathbb{R}}(M)$.

A state is a probability measure on $(M, \mathcal{F})$ and $\mathcal{S}$ denotes the set of all states. The expectation value of an observable $f$ w.r.t. the state $\nu$ is denoted by

$$
\nu(f)=\int_{M} f \mathrm{~d} \nu .
$$

If $\mathbf{f}=\left(f_{1}, \ldots, f_{N}\right)$ is a vector-valued observable we set $\nu(\mathbf{f})=\left(\nu\left(f_{1}\right), \ldots, \nu\left(f_{N}\right)\right)$. We shall equip $\mathcal{S}$ with the weakest topology w.r.t. which the functionals $\mathcal{S} \ni \nu \mapsto \nu(f)$ are continuous for all $f \in B(M)$. If $\theta: M \rightarrow M$ is a measurable map, we denote by $\nu \circ \theta^{-1}$ the measure $\mathcal{F} \ni A \mapsto \nu\left(\theta^{-1}(A)\right)$. Clearly $\nu \circ \theta^{-1}(f)=\nu(f \circ \theta)$ for all $f \in B(M)$. A map $\theta: M \rightarrow M$ is called involutive if $\theta \circ \theta(x)=x$ for all $x \in M$. 
We shall say that a state $\nu$ is normal w.r.t. $\omega \in \mathcal{S}$ iff $\nu$ is absolutely continuous w.r.t. $\omega$ (denoted $\nu \ll \omega$ ). The set of all states which are normal w.r.t. $\omega$ is denoted $\mathcal{N}_{\omega}$. Two states $\nu$ and $\omega$ are called equivalent iff $\nu \ll \omega$ and $\omega \ll \nu$, i.e., iff $\nu$ and $\omega$ have the same sets of measure zero. The Radon-Nikodym derivative $\mathrm{d} \nu / \mathrm{d} \omega$, which will play an important role in this paper, is defined as an element of $L^{1}(M, \mathrm{~d} \omega)$ and is an equivalence class of functions rather than a single function. For this reason the notion of observable is extended as follows. Given a state $\omega$, let $Z_{\omega}=$ $\{f \in \mathcal{O}(M) \mid f(x)=0$ for $\omega$-a.e. $x\}$ and let $\mathcal{O}(M)_{\omega}=\mathcal{O}(M) / Z_{\omega}$ be the quotient vector space (the elements of $\mathcal{O}(M)_{\omega}$ are equivalence classes w.r.t. the relation $\left.f \sim g \Leftrightarrow f-g \in Z_{\omega}\right)$. Similarly, $L^{\infty}(M, \mathrm{~d} \omega)=B(M) / Z_{\omega}$. As usual in measure theory, dealing with equivalence classes instead of single functions is natural and causes no difficulties, the classes are called functions, etc.

In what follows, we adopt the shorthands

$$
\Delta_{\nu \mid \omega}=\frac{\mathrm{d} \nu}{\mathrm{d} \omega}, \quad \ell_{\nu \mid \omega}=\log \Delta_{\nu \mid \omega}
$$

\subsection{Relative entropies}

The relative entropy of a state $\nu$ w.r.t. a state $\omega$ is defined by

$$
\operatorname{Ent}(\nu \mid \omega)= \begin{cases}-\infty & \text { if } \nu \notin \mathcal{N}_{\omega}, \\ -\nu\left(\ell_{\nu \mid \omega}\right) & \text { if } \nu \in \mathcal{N}_{\omega} .\end{cases}
$$

Since $-\ell_{\nu \mid \omega} \leq \Delta_{\nu \mid \omega}^{-1}-1$ and $\nu\left(\Delta_{\nu \mid \omega}^{-1}\right)=1$, relative entropy is well defined as a map from $\mathcal{S} \times \mathcal{S}$ to $[-\infty, 0]$. Its

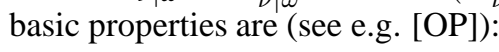

Theorem 2.1 (1) For $\omega, \nu \in \mathcal{S}$, $\operatorname{Ent}(\nu \mid \omega)=\inf _{f \in B_{\mathbb{R}}(M)}\left[\log \omega\left(\mathrm{e}^{f}\right)-\nu(f)\right]$.

(2) For $\omega \in \mathcal{S}$ and $f \in B_{\mathbb{R}}(M), \log \omega\left(\mathrm{e}^{f}\right)=\sup _{\nu \in \mathcal{S}}[\operatorname{Ent}(\nu \mid \omega)+\nu(f)]$.

(3) Concavity: For $\omega_{1}, \omega_{2}, \nu_{1}, \nu_{2} \in \mathcal{S}$ and $\lambda \in[0,1]$,

$$
\operatorname{Ent}\left(\lambda \nu_{1}+(1-\lambda) \nu_{2} \mid \lambda \omega_{1}+(1-\lambda) \omega_{2}\right) \geq \lambda \operatorname{Ent}\left(\nu_{1} \mid \omega_{1}\right)+(1-\lambda) \operatorname{Ent}\left(\nu_{2} \mid \omega_{2}\right) .
$$

(4) $\operatorname{Ent}(\nu \mid \omega) \leq 0$ for all $\omega, \nu \in \mathcal{S}$ and $\operatorname{Ent}(\nu \mid \omega)=0$ if and only if $\nu=\omega$.

(5) If $\theta: M \rightarrow M$ is a measurable bijection, then $\operatorname{Ent}\left(\nu \circ \theta^{-1} \mid \omega \circ \theta^{-1}\right)=\operatorname{Ent}(\nu \mid \omega)$.

(6) The relative entropy is an upper semicontinuous map from $\mathcal{S} \times \mathcal{S}$ to $[-\infty, 0]$, that is

$$
\operatorname{Ent}(\nu \mid \omega) \geq \limsup _{\alpha} \operatorname{Ent}\left(\nu_{\alpha} \mid \omega_{\alpha}\right),
$$

for all convergent nets $\nu_{\alpha} \rightarrow \nu$ and $\omega_{\alpha} \rightarrow \omega$ in $\mathcal{S}$.

(7) For any $\omega \in \mathcal{S}$ and any finite constant $C$, the set $\{\nu \in \mathcal{S} \mid \operatorname{Ent}(\nu \mid \omega) \geq C\}$ is compact in $\mathcal{S}$.

The Rényi relative entropy of order $\alpha \in \mathbb{R},[\mathbb{R e}]$, is defined by

$$
\operatorname{Ent}_{\alpha}(\nu \mid \omega)= \begin{cases}-\infty & \text { if } \nu \notin \mathcal{N}_{\omega} \\ \log \omega\left(\Delta_{\nu \mid \omega}^{\alpha}\right) & \text { if } \nu \in \mathcal{N}_{\omega} .\end{cases}
$$

This generalization of relative entropy has found numerous applications (see [BS,, $\mathrm{OP}]$ for references and additional information). We list below several properties of the Rényi relative entropy that are relevant for our purposes: 
Proposition 2.2 Suppose that $\nu \in \mathcal{N}_{\omega}$.

(1) If $\theta: M \rightarrow M$ is a measurable bijection, then $\operatorname{Ent}_{\alpha}\left(\nu \circ \theta^{-1} \mid \omega \circ \theta^{-1}\right)=\operatorname{Ent}_{\alpha}(\nu \mid \omega)$.

(2) $\left.\left.\mathbb{R} \ni \alpha \mapsto \operatorname{Ent}_{\alpha}(\nu \mid \omega) \in\right]-\infty, \infty\right]$ is a convex function. It is real analytic and non-positive on $] 0,1[$. It is positive for $\alpha \notin[0,1]$.

(3) $\lim _{\alpha \uparrow 1} \frac{1}{1-\alpha} \operatorname{Ent}_{\alpha}(\nu \mid \omega)=\operatorname{Ent}(\nu \mid \omega)$.

In the remaining statements we assume that $\nu$ and $\omega$ are equivalent.

(4) $\operatorname{Ent}_{0}(\nu \mid \omega)=\operatorname{Ent}_{1}(\nu \mid \omega)=0$.

(5) $\operatorname{Ent}_{\alpha}(\nu \mid \omega)=\operatorname{Ent}_{1-\alpha}(\omega \mid \nu)$.

(6) $\operatorname{Ent}_{\alpha}(\nu \mid \omega) \geq \alpha \operatorname{Ent}(\omega \mid \nu)$.

\subsection{Dynamics}

Let $\mathcal{I}$ be an index set whose elements are interpreted as instances of time. We shall always assume that $\mathcal{I}=\mathbb{Z}$ (the discrete time case) or $\mathcal{I}=\mathbb{R}$ (the continuous time case). A dynamics $\phi=\left\{\phi^{t} \mid t \in \mathcal{I}\right\}$ on $M$ is a group of invertible measurable transformations $\phi^{t}: M \rightarrow M$ describing the evolution of the system. More precisely, we shall assume:

(F1) $\phi^{0}$ is the identity map and $\phi^{t+s}=\phi^{t} \circ \phi^{s}$ for all $s, t \in \mathcal{I}$. In particular, for all $t \in \mathcal{I}, \phi^{t}$ is an automorphism of the measurable space $(M, \mathcal{F})$.

(F2) The map $(t, x) \mapsto \phi^{t}(x)$ is measurable.

The assumption (F2) is relevant only in the case $\mathcal{I}=\mathbb{R}$ (in this case, the dynamics $\phi$ is a flow on $M$ ). In the discrete time case $\mathcal{I}=\mathbb{Z}$, the dynamics is obtained by iterating the time 1 map $\phi=\phi^{1}$ and its inverse $\phi^{-1}$. We will sometimes write $\phi^{n}$ instead of $\phi^{t}$.

A dynamics $\phi$ on $M$ induces transformation groups on $\mathcal{O}(M)$ and $\mathcal{S}$ by $f_{t}=f \circ \phi^{t}, \nu_{t}=\nu \circ \phi^{-t}$. They are clearly related by $\nu_{t}(f)=\nu\left(f_{t}\right)$. A state $\nu$ is called steady (or stationary) if $\nu_{t}=\nu$ for all $t$. We denote by $\mathcal{S}_{I}$ the set of all steady states.

\subsection{Reference state}

The starting point of our discussion is a classical dynamical system $(M, \phi, \omega)$, where $\phi$ is a given dynamics on $M$ and $\omega$ a given reference state satisfying the following regularity assumption:

(C) $\omega_{t}$ and $\omega$ are equivalent for all $t \in \mathcal{I}$.

In non-trivial models that arise in non-equilibrium statistical mechanics $\mathcal{N}_{\omega} \cap \mathcal{S}_{I}=\emptyset$. In particular, $\omega \notin \mathcal{S}_{I}$. In this important aspect our starting point differs from the usual one in the ergodic theory of classical dynamical systems where the reference state $\omega$ is assumed to be invariant under the dynamics.

Assumption $(\mathrm{C})$ ensures that $\phi$ preserves $Z_{\omega}$ and hence naturally induces a group of transformations of $\mathcal{O}(M)_{\omega}$ and $L^{\infty}(M, \mathrm{~d} \omega)$.

Assumptions (F1), (F2) and (C) are our fundamental working hypothesis and will be assumed in the following without further notice. 


\subsection{Time reversal invariance}

A time reversal of the dynamics $\phi$ on $M$ is an involutive measurable transformation $\vartheta: M \rightarrow M$ such that $\vartheta \circ \phi^{t}=\phi^{-t} \circ \vartheta$ for all $t \in \mathcal{I}$.

A state $\omega \in \mathcal{S}$ is called time reversal invariant (TRI) if $\omega \circ \vartheta=\omega$. In this case $\vartheta$ preserves $Z_{\omega}$ and induces an involution on $\mathcal{O}(\mathcal{M})_{\omega}$ and $L^{\infty}(M, \mathrm{~d} \omega)$. Note that if $\omega$ is TRI, then $\omega_{t} \circ \vartheta=\omega_{-t}$.

The dynamical system $(M, \phi, \omega)$ is called TRI if $M$ is equipped with a time reversal $\vartheta$ of $\phi$ such that $\omega$ is TRI.

Time-reversal invariance will play a central role in our discussion. Other symmetries can have important consequences on statistical properties of the dynamics, e.g. the conformally symplectic structure of some systems leads to symmetries in their Lyapunov spectrum (see [WL, MD]). Such symmetries, however, will not play a role in our work.

If the system $(M, \phi, \omega)$ is not TRI, for the purpose of model building the following construction is useful. Set

$$
\widetilde{M}=M \times M, \quad \widetilde{\phi}^{t}(x, y)=\left(\phi^{t}(x), \phi^{-t}(y)\right), \quad \mathrm{d} \widetilde{\omega}=\mathrm{d} \omega \otimes \mathrm{d} \omega .
$$

Then $(\widetilde{M}, \widetilde{\phi}, \widetilde{\omega})$ is TRI with the time reversal $\vartheta(x, y)=(y, x)$

\section{Finite time entropy production}

\subsection{Entropy cocycle}

Since $\omega_{t}$ and $\omega$ are equivalent measures,

$$
c^{t}=\ell_{\omega_{t} \mid \omega} \circ \phi^{t} \in \mathcal{O}(M)_{\omega}
$$

is well-defined. It satisfies the following additive cocycle property:

Proposition 3.1 For all $t, s \in \mathcal{I}$ one has

$$
c^{t+s}=c^{s}+c^{t} \circ \phi^{s}
$$

In particular $c^{0}=0$ and $c^{-t}=-c^{t} \circ \phi^{-t}$.

Proof. We adopt the shorthand $\Delta^{t}=\Delta_{\omega_{t} \mid \omega}$. For $f \in B(M)$ and $s, t \in \mathcal{I}$ one has $\omega_{t+s}(f)=\omega\left(\Delta^{t+s} f\right)$ and

$$
\omega_{t+s}(f)=\omega_{s}\left(f_{t}\right)=\omega\left(\Delta^{s} f_{t}\right)=\omega\left(\left(\Delta^{s} \circ \phi^{-t} f\right) \circ \phi^{t}\right)=\omega_{t}\left(\Delta^{s} \circ \phi^{-t} f\right)=\omega\left(\Delta^{s} \circ \phi^{-t} \Delta^{t} f\right) .
$$

Hence,

$$
\Delta^{t+s}=\Delta^{s} \circ \phi^{-t} \Delta^{t}
$$

where the equality is in $\mathcal{O}(M)_{\omega}$. Taking the logarithm we derive

$$
\ell_{\omega_{t+s} \mid \omega}=\ell_{\omega_{s} \mid \omega} \circ \phi^{-t}+\ell_{\omega_{t} \mid \omega}
$$

Our first identity follows immediately. The second one follows from the substitution $s=0$ and the third one is obtained by setting $s=-t$.

We shall call $c^{t}$ the entropy cocycle of the dynamical system $(M, \phi, \omega)$. The entropy cocycle of a TRI dynamical system enjoys the following additional property. 
Proposition 3.2 If the system $(M, \phi, \omega)$ is TRI with a time reversal $\vartheta$, then

$$
c^{t} \circ \vartheta=c^{-t}
$$

holds for all $t \in \mathcal{I}$.

Proof. Setting again $\Delta^{t}=\Delta_{\omega_{t} \mid \omega}$ we have, for any $f \in B(M)$ and $t \in \mathcal{I}$,

$\omega\left(\Delta^{t} \circ \vartheta f\right)=\omega \circ \vartheta\left(\Delta^{t} f \circ \vartheta\right)=\omega\left(\Delta^{t} f \circ \vartheta\right)=\omega_{t}(f \circ \vartheta)=\omega\left(f \circ \vartheta \circ \phi^{t}\right)=\omega\left(f \circ \phi^{-t} \circ \vartheta\right)=\omega\left(f \circ \phi^{-t}\right)=\omega\left(\Delta^{-t} f\right)$.

The resulting identity $\Delta^{-t}=\Delta^{t} \circ \vartheta$ further leads to $\Delta^{-t} \circ \phi^{-t}=\Delta^{t} \circ \vartheta \circ \phi^{-t}=\Delta^{t} \circ \phi^{t} \circ \vartheta$. Taking the logarithm gives the result.

\subsection{Entropy balance equation}

By Definition (2.1) of the relative entropy one has

$$
\operatorname{Ent}\left(\omega_{t} \mid \omega\right)=-\omega_{t}\left(\ell_{\omega_{t} \mid \omega}\right)=-\omega\left(\ell_{\omega_{t} \mid \omega} \circ \phi^{t}\right)=-\omega\left(c^{t}\right) .
$$

Since $\operatorname{Ent}(\omega \mid \omega)=0$ this identity can be rewritten as

$$
\omega\left(\Sigma^{t}\right)=-\frac{1}{t}\left(\operatorname{Ent}\left(\omega_{t} \mid \omega\right)-\operatorname{Ent}(\omega \mid \omega)\right)
$$

where

$$
\Sigma^{t}=\frac{c^{t}}{t}
$$

Thus, we can interpret $\Sigma^{t}$ as the observable of mean entropy production rate over the time interval $[0, t]$. We shall call the relation

$$
\operatorname{Ent}\left(\omega_{t} \mid \omega\right)=-t \omega\left(\Sigma^{t}\right)
$$

the entropy balance equation. Its immediate consequence is the important inequality

$$
\omega\left(\Sigma^{t}\right) \in[0, \infty],
$$

which holds for all $t>0$.

The cocycle property yields

$$
\Sigma^{t}=-\frac{c^{-t} \circ \phi^{t}}{t}=\Sigma^{-t} \circ \phi^{t} .
$$

We note for later reference that if $(M, \phi, \omega)$ is TRI, then Proposition 3.2 further leads to

$$
\Sigma^{t} \circ \vartheta=\frac{c^{-t}}{t}=-\Sigma^{-t}=-\Sigma^{t} \circ \phi^{-t} .
$$

\subsection{Finite time Evans-Searles symmetry}

Let $P_{t}$ be the law of the real-valued random variable $\Sigma_{t}$, i.e., the Borel probability measure on $\mathbb{R}$ such that for any $f \in B(\mathbb{R})$,

$$
P_{t}(f)=\omega\left(f\left(\Sigma^{t}\right)\right)
$$

Let $\mathfrak{r}: \mathbb{R} \rightarrow \mathbb{R}$ be the reflection $\mathfrak{r}(s)=-s$ and define the reflected measure $\bar{P}_{t}=P_{t} \circ \mathfrak{r}$. 
Proposition 3.3 If $(M, \phi, \omega)$ is TRI then, for any $t \in \mathcal{I}$, the measures $P_{t}$ and $\bar{P}_{t}$ are equivalent and

$$
\frac{\mathrm{d} \bar{P}_{t}}{\mathrm{~d} P_{t}}(s)=\mathrm{e}^{-t s} .
$$

Proof. For $f \in B(\mathbb{R})$, Equ. (3.8) and the fact that $\omega_{t} \circ \vartheta=\omega_{-t}$ yield

$$
\bar{P}_{t}(f)=\omega\left(f\left(-\Sigma^{t}\right)\right)=\omega_{t}\left(f\left(-\Sigma^{t} \circ \phi^{-t}\right)\right)=\omega_{t}\left(f\left(\Sigma^{t} \circ \vartheta\right)\right)=\omega_{-t}\left(f\left(\Sigma^{t}\right)\right)=\omega\left(\mathrm{e}^{-t \Sigma^{t}} f\left(\Sigma^{t}\right)\right),
$$

and the statement follows.

To our knowledge, the relation (3.9) was first obtained by Evans and Searles in [ES] and is sometimes called the transient fluctuation theorem. We shall call it the finite time ES-identity. We stress its universal character: besides the TRI assumption it only relies on the minimal hypothesis (F1), (F2) and (C), In a loose sense, it can be understood as a dynamical form of the second law of thermodynamics: on the finite time interval $[0, t]$, the probability to observe a negative mean entropy production rate $-s$ is exponentially small compared to the probability to observe the positive value $s$.

The ES-identity can be re-formulated in terms of Rényi entropy. For $\alpha \in \mathbb{R}$, we adopt the shorthand

$$
e_{t}(\alpha)=\operatorname{Ent}_{\alpha}\left(\omega_{t} \mid \omega\right)=\log \omega\left(\mathrm{e}^{\alpha \ell_{\omega_{t} \mid \omega}}\right)=\log \omega\left(\mathrm{e}^{\alpha t \Sigma^{-t}}\right) .
$$

By Theorem 2.1 (2), if $\ell_{\omega_{t} \mid \omega} \in L^{\infty}(M, \mathrm{~d} \omega)$, then

$$
e_{t}(\alpha)=\sup _{\nu \in \mathcal{N}_{\omega}}\left[\operatorname{Ent}(\nu \mid \omega)+\alpha \nu\left(\ell_{\omega_{t} \mid \omega}\right)\right] .
$$

This variational characterization will play an important role in the extension of the theory of entropic fluctuations to non-commutative dynamical systems.

The basic properties of the functional (3.10) follow directly from Proposition 2.2. We list them for later reference:

Proposition 3.4 (1) For all $t \in \mathcal{I}$ the function

$$
\left.\left.\mathbb{R} \ni \alpha \mapsto e_{t}(\alpha) \in\right]-\infty, \infty\right]
$$

is convex, satisfies $e_{t}(0)=e_{t}(1)=0$ and

$$
\begin{cases}\left.\left.e_{t}(\alpha) \in\right]-\infty, 0\right] & \text { if } \alpha \in[0,1] \\ e_{t}(\alpha) \in[0, \infty] & \text { otherwise. }\end{cases}
$$

(2) It satisfies the lower bound

$$
e_{t}(\alpha) \geq \min \left(\alpha \operatorname{Ent}\left(\omega \mid \omega_{t}\right),(1-\alpha) \operatorname{Ent}\left(\omega_{t} \mid \omega\right)\right) .
$$

(3) It is real analytic on the interval $] 0,1[$.

Remark. The relation $e_{t}(1)=0$ is sometimes called the non-equilibrium partition identity or Kawasaki identity, see [CWW].

Note that if the system is TRI, Equ. (3.8) implies $\omega\left(\mathrm{e}^{\alpha t \Sigma^{-t}}\right)=\omega\left(\mathrm{e}^{-\alpha t \Sigma^{t} \circ \vartheta}\right)=\omega\left(\mathrm{e}^{-\alpha t \Sigma^{t}}\right)$ so that

$$
e_{t}(\alpha)=\log \omega\left(\mathrm{e}^{-\alpha t \Sigma^{t}}\right) .
$$


Proposition 3.5 (1) For any $t \in \mathcal{I}$ and $\alpha \in \mathbb{R}$ one has $e_{t}(\alpha)=e_{-t}(1-\alpha)$.

(2) If $(M, \phi, \omega)$ is TRI then $e_{-t}(\alpha)=e_{t}(\alpha)$ and hence

$$
e_{t}(\alpha)=e_{t}(1-\alpha) \text {. }
$$

Proof. Parts (1) and (5) of Proposition 2.2 imply

$$
e_{t}(\alpha)=\operatorname{Ent}_{\alpha}\left(\omega_{t} \mid \omega\right)=\operatorname{Ent}_{1-\alpha}\left(\omega \mid \omega_{t}\right)=\operatorname{Ent}_{1-\alpha}\left(\omega_{-t} \mid \omega\right)=e_{-t}(1-\alpha) .
$$

Since TRI implies $\omega_{t} \circ \vartheta=\omega_{-t}$, Part (1) of Proposition 2.2 allows us to conclude

$$
e_{-t}(\alpha)=\operatorname{Ent}_{\alpha}\left(\omega_{-t} \mid \omega\right)=\operatorname{Ent}_{\alpha}\left(\omega_{t} \circ \vartheta \mid \omega \circ \vartheta\right)=\operatorname{Ent}_{\alpha}\left(\omega_{t} \mid \omega\right)=e_{t}(\alpha) .
$$

We shall call Relation (3.13) the finite time ES-symmetry. We finish this section with the observation that the finite time ES-symmetry is an equivalent formulation of the finite time ES-identity.

Proposition 3.6 For each $t \in \mathcal{I}$, the following statements are equivalent:

(1) The measures $P_{t}$ and $\bar{P}_{t}$ are equivalent and satisfy the ES-identity (3.9).

(2) For all $\alpha \in \mathbb{R}, e_{-t}(\alpha)=e_{-t}(1-\alpha)$.

Proof. It suffices to notice the relation between the functional $e_{-t}(\alpha)$ and the Laplace transform of the measure $P_{t}$. One has

$$
e_{-t}(\alpha)=\log \omega\left(\mathrm{e}^{-\alpha t \Sigma^{t}}\right)=\log \int \mathrm{e}^{\alpha t s} \mathrm{~d} \bar{P}_{t}(s)
$$

and hence

$$
e_{-t}(1-\alpha)=\log \int \mathrm{e}^{-(1-\alpha) t s} \mathrm{~d} P_{t}(s)=\log \int \mathrm{e}^{\alpha t s} \mathrm{e}^{-t s} \mathrm{~d} P_{t}(s)
$$

\subsection{Entropy production observable}

For a discrete time dynamical system the cocycle property $c^{t+1}=c^{t}+c^{1} \circ \phi^{t}$ implies

$$
c^{t}=\sum_{s=0}^{t-1} \sigma_{s},
$$

where

$$
\sigma=c^{1}=\ell_{\omega_{1} \mid \omega} \circ \phi .
$$

In particular, we can express the mean entropy production rate observable as

$$
\Sigma^{t}=\frac{c^{t}}{t}=\frac{1}{t} \sum_{s=0}^{t-1} \sigma_{s} .
$$


Consequently, the entropy balance equation (3.6) becomes

$$
\operatorname{Ent}\left(\omega_{t} \mid \omega\right)=-\sum_{s=0}^{t-1} \omega\left(\sigma_{s}\right) .
$$

We shall call $\sigma$ the entropy production observable. For obvious reasons the entropy production observable is also often called phase space contraction rate.

Basic properties of the entropy production observable are:

Proposition 3.7 $\omega(\sigma) \geq 0, \omega_{-1}(\sigma) \leq 0$, and if $(M, \phi, \omega)$ is [TRI then $\sigma \circ \vartheta=-\sigma_{-1}$.

Proof. $-\omega(\sigma)=-\omega_{1}\left(\ell_{\omega_{1} \mid \omega}\right)=\operatorname{Ent}\left(\omega_{1} \mid \omega\right) \leq 0$ implies $\omega(\sigma) \geq 0$. Jensen's inequality

$$
\mathrm{e}^{\omega_{-1}(\sigma)} \leq \omega_{-1}\left(\mathrm{e}^{\sigma}\right)=\omega\left(\Delta_{\omega_{1} \mid \omega}\right)=1,
$$

implies $\omega_{-1}(\sigma) \leq 0$. The last statement follows from (3.4) and the cocycle property $c^{-1}=-c^{1} \circ \phi^{-1}$.

It is not possible to define the entropy production observable of a continuous time dynamical system at the current level of generality. We shall make some minimal regularity assumptions to ensure that the entropy cocycle has a generator $\sigma$, i.e., that the continuous time analog of Equ. (3.14) holds.

(E1) 1 . The function $\mathbb{R} \ni t \mapsto \Delta_{\omega_{t} \mid \omega} \in L^{1}(M, \mathrm{~d} \omega)$ is strongly $C^{1}$.

2. The entropy production observable

$$
\sigma=\left.\frac{\mathrm{d}}{\mathrm{d} t} \Delta_{\omega_{t} \mid \omega}\right|_{t=0}
$$

is such that the function $\mathbb{R} \ni t \mapsto \sigma_{t} \in L^{1}(M, \mathrm{~d} \omega)$ is strongly continuous.

Remark. If $M$ is a complete, separable and metrizable space, then the Koopman operators $T^{t}: f \mapsto \Delta_{\omega_{t} \mid \omega} f_{-t}$ form a strongly continuous group of isometries of $L^{1}(M, \mathrm{~d} \omega)$. Denote by $L$ its generator, i.e., $T^{t}=\mathrm{e}^{t L}$. Since $\Delta_{\omega_{t} \mid \omega}=T^{t} 1$, Part 1 of Assumption (E1) is equivalent to $1 \in \operatorname{Dom}(L)$, the domain of $L$, and then $\sigma=L 1$.

Proposition 3.8 Suppose that (E1) holds. Then:

(1) For all $x \in M$ the function $t \mapsto \Delta_{\omega_{t} \mid \omega}(x)$ is absolutely continuous and

$$
\frac{\mathrm{d}}{\mathrm{d} t} \Delta_{\omega_{t} \mid \omega}(x)=\Delta_{\omega_{t} \mid \omega}(x) \sigma_{-t}(x),
$$

holds for $\omega$-almost all $x \in M$ and Lebesgue almost all $t \in \mathbb{R}$.

(2) For all $t \in \mathbb{R}$ the identities

$$
\Delta_{\omega_{t} \mid \omega}=\mathrm{e}^{\int_{0}^{t} \sigma_{-s} \mathrm{~d} s},
$$

and

$$
c^{t}=\int_{0}^{t} \sigma_{s} \mathrm{~d} s,
$$

hold in $\mathcal{O}(M)_{\omega}$.

(3) $\omega(\sigma)=0$. 
(4) If $(M, \phi, \omega)$ is TRI then $\sigma \circ \vartheta=-\sigma$.

Proof. We again set $\Delta^{t}=\Delta_{\omega_{t} \mid \omega}$.

(1) The cocycle property (3.3) and Part (1) of Assumption (E1) yield

$$
\omega\left(\left|\Delta^{t+s}-\Delta^{t}-s \Delta^{t} \sigma_{-t}\right|\right)=\omega\left(\Delta^{t}\left|\Delta^{s} \circ \phi^{-t}-1-s \sigma_{-t}\right|\right)=\omega\left(\left|\Delta^{s}-1-s \sigma\right|\right)=o(s),
$$

as $s \rightarrow 0$, from which we conclude that

$$
\frac{\mathrm{d}}{\mathrm{d} t} \Delta^{t}=\Delta^{t} \sigma_{-t}
$$

holds strongly in $L^{1}(M, \mathrm{~d} \omega)$. By Part (1) of Assumption [E1) we can assume that for any $x \in M$ the function $t \mapsto \Delta^{t}(x)$ is absolutely continuous and that for $\omega$-almost all $x \in M$

$$
\frac{\mathrm{d}}{\mathrm{d} t} \Delta^{t}(x)=\Delta^{t}(x) \sigma_{-t}(x),
$$

holds for Lebesgue almost all $t \in \mathbb{R}$ (see e.g. Theorem 3.4.2 in [ [HP]).

(2) By Part (2) of Assumption (E1), the Riemann integral

$$
\ell_{t}=\int_{0}^{s} \sigma_{-s} \mathrm{~d} s
$$

defines a strongly $C^{1}$ function $t \mapsto \ell_{t} \in L^{1}(M, \mathrm{~d} \omega)$. As before, we can assume that $t \mapsto \ell_{t}(x)$ is absolutely continuous for all $x \in M$ and that for $\omega$-almost all $x \in M$

$$
\frac{\mathrm{d}}{\mathrm{d} t} \ell_{t}(x)=\sigma_{-t}(x)
$$

holds for Lebesgue almost all $t \in \mathbb{R}$. Consequently, for all $x \in M$ the function $t \mapsto F_{t}(x)=\Delta^{t}(x) \mathrm{e}^{-\ell_{t}(x)}$ is absolutely continuous and for $\omega$-almost all $x \in M$

$$
\frac{\mathrm{d}}{\mathrm{d} t} F_{t}(x)=0,
$$

holds for Lebesgue almost all $t \in \mathbb{R}$. We conclude that for all $t \in \mathbb{R}, F_{t}(x)=F_{0}(x)=1$, i.e., $\Delta^{t}(x)=\mathrm{e}^{\ell_{t}(x)}$ for $\omega$-almost all $x \in M$. Equ. (3.16) follows immediately.

(3) Differentiating the identity $e_{t}(1)=0$ w.r.t. $t$ at $t=0$ we derive $\omega(\sigma)=0$.

(4) Follows from the identity (3.4).

Remark. Under the strong continuity condition of Assumption (E1), the identity (3.16) holds in $\mathcal{O}(M)_{\omega}$ with a Lebesgue integral. It also holds in $L^{1}(M, \mathrm{~d} \omega)$ with a strong Riemann integral.

The relation (3.16) yields

$$
\Sigma_{t}=\frac{1}{t} \int_{0}^{t} \sigma_{s} \mathrm{~d} s
$$

and so the entropy balance equation and the ES-functional can be written as

$$
\operatorname{Ent}\left(\omega_{t} \mid \omega\right)=-\int_{0}^{t} \omega\left(\sigma_{s}\right) \mathrm{d} s
$$




$$
e_{t}(\alpha)=\log \omega\left(\mathrm{e}^{\alpha \int_{0}^{t} \sigma_{-s} \mathrm{~d} s}\right)
$$

For TRI systems one has $\sigma_{-s} \circ \vartheta=-\sigma_{s}$ and in this case

$$
e_{t}(\alpha)=\log \omega\left(\mathrm{e}^{-\alpha \int_{0}^{t} \sigma_{s} \mathrm{~d} s}\right)
$$

Unless otherwise stated, in the sequel we will only consider continuous time dynamical systems. The discrete time case is very similar, time-integrals being replaced by appropriate sums.

\section{5 $L^{p}$-Liouvilleans}

In this section, in order to avoid unessential technicalities, we shall assume in addition to (E1).

(E2) $\sigma \in L^{\infty}(M, \mathrm{~d} \omega)$.

Let $p \in]-\infty, \infty], p \neq 0$, and $f \in L^{\infty}(M, \mathrm{~d} \omega)$. We shall consider the following special class of Ruelle transfer operators

$$
U_{p}(t) f=\Delta_{\omega_{t} \mid \omega}^{\frac{1}{p}} f_{-t}=\mathrm{e}^{\frac{1}{p} \int_{0}^{t} \sigma_{-s} \mathrm{~d} s} f_{-t}
$$

One easily shows:

Proposition 3.9 Under Assumptions (E1) (E2). Equ. (3.17) defines a family of bounded linear operators on $L^{\infty}(M, \mathrm{~d} \omega)$ which satisfies:

(1) $U_{p}(0)=I$ and $U_{p}(t+s)=U_{p}(t) U_{p}(s)$.

(2) If $p^{-1}+q^{-1}=1$, then $\omega\left(\left[U_{p}(t) f\right]\left[U_{q}(t) g\right]\right)=\omega(f g)$.

(3) $\omega\left(\left|U_{p}(t) f\right|^{p}\right)=\omega\left(|f|^{p}\right)$. For $p \in[1, \infty], U_{p}(t)$ extends to a group of isometries of $L^{p}(M, \mathrm{~d} \omega)$.

(4) $U_{p}(t)$ extends to a group of bounded operators on $L^{2}(M, \mathrm{~d} \omega)$ such that $U_{p}^{*}(t)=U_{q}(-t)$ and

$$
\left\|U_{p}(t)\right\| \leq \mathrm{e}^{|t| m_{p}}, \quad m_{p}=\frac{|2-p|}{|p|}\|\sigma\|_{\infty} .
$$

(5) Suppose that $U_{p}(t)$ is strongly continuous on $L^{2}(M, \mathrm{~d} \omega)$ and let $L_{p}$ be its generator, $U_{p}(t)=\mathrm{e}^{t L_{p}}$. Then $L_{p}^{*}=-L_{q}, \operatorname{sp}\left(L_{p}\right) \subset\left\{z|| \operatorname{Re} z \mid \leq m_{p}\right\}, \operatorname{Dom}\left(L_{p}\right)=\operatorname{Dom}\left(L_{\infty}\right)$, and for $f \in \operatorname{Dom}\left(L_{p}\right)$

$$
L_{p} f=L_{\infty} f+\frac{\sigma}{p} f .
$$

We shall call the operator $L_{p}$ the $L^{p}$-Liouvillean. In Part (5), $\operatorname{Dom}(A)$ denotes the domain of the operator $A$ and $\operatorname{sp}(A)$ its spectrum.

If $\alpha=1 / p$, then

$$
e_{t}(\alpha)=\log \left(1, \mathrm{e}^{t L_{p}} 1\right)=\log \int_{M} \mathrm{e}^{t L_{p}} 1 \mathrm{~d} \omega .
$$

Similarly to (3.11), this operator characterization of $e_{t}(\alpha)$ will play an important role in the extension of the theory of entropic fluctuations to the non-commutative setting. 
If $\sigma$ is unbounded, the operator $U_{p}(t)$ could be unbounded and Proposition 3.9 may not hold as formulated. The technical aspects of its extension are then best carried out in the context of concrete models.

In the discrete time case the concept of $L^{p}$-Liouvillean is not very natural and instead one deals directly with the transfer operator

$$
U_{p} f=\mathrm{e}^{\frac{1}{p} \sigma} f_{-1} .
$$

If $\sigma \in L^{\infty}(M, \mathrm{~d} \omega)$, then Parts (1)-(4) of Proposition 3.9 obviously hold, $U_{p}(n)=U_{p}^{n}$ and $e_{n}(\alpha)=\log \left(1, U_{p}^{n} 1\right)$.

\subsection{Examples: Differentiable dynamics and thermostated systems}

Let $U \subset \mathbb{R}^{n}$ be an open connected set and let $\phi: U \rightarrow U$ be a $C^{1}$-diffeomorphism. Denote by $D \phi$ its derivative. Let $M \subset U$ be compact and suppose that $\phi(M) \subset M$. Finally denote by $\omega$ the normalized Lebesgue measure on $M$. The entropy production observable of the discrete time dynamical system $(M, \phi, \omega)$ is given by

$$
\sigma=-\left.\log |\operatorname{det} D \phi|\right|_{M} .
$$

To describe the continuous time case let $X$ be a $C^{1}$-vector field on $U$. Assume that the flow $\phi$ generated by the differential equation

$$
\frac{\mathrm{d}}{\mathrm{d} t} x_{t}=X\left(x_{t}\right),
$$

satisfies $\phi^{t}(M) \subset M$. Then $\phi$ is $C^{1}$ on $\mathbb{R} \times M$. The entropy cocycle of $(M, \phi, \omega)$ is $c^{t}=-\log \mid \operatorname{det} D \phi^{t} \|_{M}$ and the entropy production observable is given by

$$
\sigma=-\left.\frac{\mathrm{d}}{\mathrm{d} t} \log \left|\operatorname{det} D \phi^{t}\right|\right|_{M, t=0}=-\left.\operatorname{div} X\right|_{M} .
$$

Assumptions (E1) (E2) are clearly satisfied.

A special class of differentiable dynamics is provided by the so called Gaussian thermostated systems. Consider a Hamiltonian system with $n$ degrees of freedom. The phase space of the system is $\mathbb{R}^{n} \oplus \mathbb{R}^{n}$ (or more generally the cotangent bundle of a smooth manifold). For simplicity, we shall assume that its Hamiltonian $H$ is $C^{2}$ and that the finite energy subsets $\{(p, q) \mid H(q, p) \leq E\}$ are compact. These assumptions ensure that the equations of motion

$$
\dot{p}_{t}=-\nabla_{q} H\left(p_{t}, q_{t}\right), \quad \dot{q}_{t}=\nabla_{p} H\left(p_{t}, q_{t}\right),
$$

define a global $C^{1}$ Hamiltonian flow $\phi_{H}^{t}(p, q)=\left(p_{t}, q_{t}\right)$ which preserves the energy, $H \circ \phi_{H}^{t}=H$, and Lebesgue measure on $\mathbb{R}^{n} \oplus \mathbb{R}^{n}$ (Liouville's theorem).

To drive this system out of equilibrium, an external non-Hamiltonian force $F(q)$ is applied. To prevent it from heating up, the energy supplied by this force is removed by a thermostat (the so-called Gaussian thermostat). This leads to the modified equations of motion

$$
\dot{p}_{t}=-\nabla_{q} H\left(p_{t}, q_{t}\right)+F\left(q_{t}\right)-\Theta\left(q_{t}, p_{t}\right), \quad \dot{q}_{t}=\nabla_{p} H\left(p_{t}, q_{t}\right),
$$

where the thermostating force is given by

$$
-\Theta(p, q)=-\frac{F(q) \cdot \nabla_{p} H(p, q)}{\left|\nabla_{p} H(p, q)\right|^{2}} \nabla_{p} H(p, q) .
$$

One easily checks that the flow $\phi$ generated by this system satisfies $H \circ \phi^{t}=H$ and therefore preserves the total energy. This flow, however, does not preserve Lebesgue measure and the entropy production observable

$$
\sigma=\nabla_{p} \cdot \Theta(q, p)
$$


measures the local rate of phase space contraction.

Fixing $E \in \operatorname{Ran} H$ we see that Gaussian thermostated systems are special cases of differentiable dynamics with

$$
M=\left\{(p, q) \in \mathbb{R}^{n} \oplus \mathbb{R}^{n} \mid H(p, q)=E\right\}
$$

Two other well-known thermostating mechanisms are the isokinetic and Nosé-Hoover thermostats. Models using these thermostats have been constructed to describe various phenomena like shear flows [ECM], CL], heat conduction [HHP, $\mathrm{PH}]$, and turbulent fluids [Ga3, GRS]. They all have in common that the dynamics is described by a deterministic finite-dimensional dynamical system on a compact manifold and are very convenient for numerical studies [Ho, EM, Do].

A well-known model in this class is a Sinai billiard with an external electric field [CELS1, CELS2, Ch1, Ch2, Yo, RY]. General mathematical results concerning thermostated Hamiltonian models can be found in [GC1, GC2, $\mathrm{Ga1}, \mathrm{Ga} 2, \mathrm{Ru} 2, \mathrm{Ru} 3, \mathrm{Ru} 4, \mathrm{Ru} 5$.

\subsubsection{A micro-canonical ideal gas out of equilibrium.}

In this section we consider an exactly solvable thermostated system - a gas of $N>1$ identical, non-interacting particles moving on a circle. The phase space is $\mathbb{R}^{N} \times \mathbb{T}^{N}$ equipped with Lebesgue measure and the Hamiltonian is

$$
H(L, \theta)=\frac{1}{2}|L|^{2}
$$

The flow $\phi$ is generated by the system

$$
\left.\begin{array}{l}
\dot{L}_{j}=F-\lambda(L) L_{j}, \\
\dot{\theta}_{j}=L_{j},
\end{array}\right\} \quad(j=1, \ldots, N) .
$$

Here, $F \in \mathbb{R}$ denotes the constant strength of the external forcing and $-\lambda(L) L_{j}$ is the thermostating force,

$$
\lambda(L)=F \frac{\ell}{u}, \quad \ell=\frac{1}{N} \sum_{k=1}^{N} L_{k}, \quad u=\frac{1}{N} \sum_{k=1}^{N} L_{k}^{2} .
$$

The mean kinetic energy per particle $u$ is constant under the flow $\phi$ and we consider the dynamical system $(M, \phi, \omega)$ where

$$
M=\{(L, \theta) \mid u=\epsilon\} \simeq S^{N-1} \times \mathbb{T}^{N},
$$

for some $\epsilon>0$ and $\omega$ is the normalized micro-canonical measure

$$
\omega(f)=\frac{1}{Z} \int_{\mathbb{R}^{N} \times \mathbb{T}^{N}} f(L, \theta) \delta(u-\epsilon) \prod_{j=1}^{N} \mathrm{~d} L_{j} \mathrm{~d} \theta_{j} .
$$

The Cauchy-Schwarz inequality ensures that $\ell^{2} \leq u$ on $M$ and the observable

$$
\xi=-\frac{1}{2} \log \left(\frac{\sqrt{u}-\ell}{\sqrt{u}+\ell}\right)
$$


is well defined. One derives $\dot{\xi}=\mu$ where $\mu=F \epsilon^{-1 / 2}$ is a constant. It follows that $\ell=\sqrt{\epsilon}$ th $\xi$ which, once inserted in (3.21), allows to integrate the equations of motion (3.20) to obtain

$$
\begin{aligned}
\theta_{j t} & =\theta_{j 0}+\frac{\sqrt{\epsilon}}{F}\left(L_{j 0} \operatorname{ch} \xi_{0}-\sqrt{\epsilon} \operatorname{sh} \xi_{0}\right)\left(\operatorname{arctg}\left(\operatorname{sh} \xi_{t}\right)-\operatorname{arctg}\left(\operatorname{sh} \xi_{0}\right)\right)+\frac{\epsilon}{F} \log \frac{\operatorname{ch} \xi_{0}}{\operatorname{ch} \xi_{t}} \\
L_{j t} & =\frac{L_{j 0} \operatorname{ch} \xi_{0}-\sqrt{\epsilon} \operatorname{sh} \xi_{0}}{\operatorname{ch} \xi_{t}}+\sqrt{\epsilon} \operatorname{th} \xi_{t}
\end{aligned}
$$

where $\xi_{t}=\xi_{0}+\mu t$. The entropy production observable is

$$
\sigma=(N-1) \frac{F}{\sqrt{\epsilon}} \operatorname{th} \xi
$$

Assumptions (E1) (E2) are satisfied in this model. Moreover, the map $\vartheta(L, \theta)=(-L, \theta)$ is a time reversal for the flow $\phi$ and the measure $\omega$ is TRI.

One easily computes

$$
\exp \left(-\alpha \int_{0}^{t} \sigma_{s} \mathrm{~d} s\right)=\left(\frac{\operatorname{ch} \xi_{0}}{\operatorname{ch} \xi_{t}}\right)^{(N-1) \alpha} .
$$

Since the distribution of $\xi$ induced by the measure $\omega$ is

$$
\omega(f(\xi))=\frac{\Gamma(N / 2)}{\sqrt{\pi} \Gamma((N-1) / 2)} \int_{-\infty}^{\infty} f(\xi)(\operatorname{ch} \xi)^{-(N-1)} \mathrm{d} \xi
$$

we conclude that

$$
e_{t}(\alpha)=\log \left(\frac{\Gamma(N / 2)}{\sqrt{\pi} \Gamma((N-1) / 2)} \int_{-\infty}^{\infty}(\operatorname{ch} \xi)^{-(N-1)(1-\alpha)}(\operatorname{ch}(\xi+\mu t))^{-(N-1) \alpha} \mathrm{d} \xi\right) .
$$

The validity of the finite time ES-symmetry, ensured by Proposition 3.5. can be explicitly checked by noticing that

$$
\begin{aligned}
\int_{-\infty}^{\infty}(\operatorname{ch} \xi)^{-(N-1)(1-\alpha)}(\operatorname{ch}(\xi+\mu t))^{-(N-1) \alpha} \mathrm{d} \xi & =\int_{-\infty}^{\infty}(\operatorname{ch}(\xi-\mu t))^{-(N-1)(1-\alpha)}(\operatorname{ch} \xi)^{-(N-1) \alpha} \mathrm{d} \xi \\
& =\int_{-\infty}^{\infty}(\operatorname{ch}(-\xi-\mu t))^{-(N-1)(1-\alpha)}(\operatorname{ch}(-\xi))^{-(N-1) \alpha} \mathrm{d} \xi \\
& =\int_{-\infty}^{\infty}(\operatorname{ch}(\xi+\mu t))^{-(N-1)(1-\alpha)}(\operatorname{ch} \xi)^{-(N-1) \alpha} \mathrm{d} \xi
\end{aligned}
$$

This example continues in Sections 5.5.1 and 6.4.1

\section{Thermodynamics}

\subsection{Basic notions}

Suppose that our dynamical system $\left(M, \phi_{X}, \omega_{X}\right)$ depends on some control parameters $X=\left(X_{1}, \cdots, X_{N}\right) \in \mathbb{R}^{N}$. One can think of the $X_{j}$ 's as mechanical or thermodynamical forces (affinities in the language of non-equilibrium thermodynamics) acting on the system. When dealing with such families of systems, we shall always assume that $(\mathrm{F} 1)(\mathrm{F} 2),(\mathrm{C})$ and (E1) hold for each system $\left(M, \phi_{X}, \omega_{X}\right)$. The entropy production observable of $\left(M, \phi_{X}, \omega_{X}\right)$ is denoted $\sigma_{X}$. We shall also assume: 
(T1) $\omega_{0}$ is $\phi_{0}^{t}$ invariant.

We will write $\phi^{t}=\phi_{0}^{t}, \omega=\omega_{0}, \omega_{0 t}=\omega_{t}$, etc, and refer to the value $X=0$ as equilibrium. Under assumption (T1) the entropy cocycle satisfies $c^{t}=c_{0}^{t}=0$ for all $t \in \mathbb{R}$ and consequently $\sigma=\sigma_{0}=0$.

Definition 4.1 We call a family of vector-valued real observables $\Phi_{X}=\left(\Phi_{X}^{(1)}, \cdots, \Phi_{X}^{(N)}\right), X \in \mathbb{R}^{N}$, a flux relation if, for all $X$,

$$
\sigma_{X}=X \cdot \boldsymbol{\Phi}_{X}=\sum_{j=1}^{N} X_{j} \Phi_{X}^{(j)} .
$$

In what follows our discussion of thermodynamics concerns a family of quadruples $\left(M, \phi_{X}, \omega_{X}, \boldsymbol{\Phi}_{X}\right)$, where $\boldsymbol{\Phi}_{X}$ is a given flux relation. In concrete models arising in physics, physical requirements typically select a unique flux relation $\boldsymbol{\Phi}_{X}$ (see Section 4.4 for an example). We will refer to $\Phi_{X}^{(j)}$ as the flux (or current) observable associated to the force $X_{j}$. Since $\sigma_{0}=0$, if the map $X \mapsto \sigma_{X}$ is smooth we can always pick the fluxes as

$$
\boldsymbol{\Phi}_{X}=\left.\int_{0}^{1} \nabla \sigma_{Y}\right|_{Y=u X} \mathrm{~d} u
$$

Remark. To simplify the notation, unless otherwise stated we shall always assume that $\left(M, \phi_{X}, \omega_{X}, \mathbf{\Phi}_{X}\right)$ is defined for all $X \in \mathbb{R}^{N}$. In concrete situations (e.g. like in the class of examples introduced in Section 4.4), the systems may only be defined on a restricted range of the physical parameters $X_{1}, \ldots, X_{N}$. This causes no difficulties-one can either trivially extend the range of parameters to all of $\mathbb{R}^{N}$ or indicate in each statement the range of parameters to which they apply.

Our second general assumption concerns time reversal.

(T2) The dynamical systems $\left(M, \phi_{X}, \omega_{X}\right)$ are time-reversal invariant and

$$
\boldsymbol{\Phi}_{X} \circ \vartheta_{X}=-\boldsymbol{\Phi}_{X} .
$$

This assumption implies that $\omega_{X}\left(\boldsymbol{\Phi}_{X}\right)=0$ for all $X$.

\subsection{Finite time Generalized Evans-Searles symmetry}

Let

$$
\boldsymbol{\Sigma}_{X}^{t}=\frac{1}{t} \int_{0}^{t} \boldsymbol{\Phi}_{X s} \mathrm{~d} s=\left(\frac{1}{t} \int_{0}^{t} \Phi_{X s}^{(1)} \mathrm{d} s, \ldots, \frac{1}{t} \int_{0}^{t} \Phi_{X s}^{(N)} \mathrm{d} s\right),
$$

where $\boldsymbol{\Phi}_{X s}=\boldsymbol{\Phi}_{X} \circ \phi_{X}^{s}, \Phi_{X s}^{(j)}=\Phi_{X}^{(j)} \circ \phi_{X}^{s}$. The entropy cocycle can be written as

$$
c_{X}^{t}=t X \cdot \Sigma_{X}^{t} .
$$

Let $P_{X}^{t}$ be the law of $\boldsymbol{\Sigma}_{X}^{t}$, i.e., the Borel probability measure on $\mathbb{R}^{N}$ such that $P_{X}^{t}(f)=\omega_{X}\left(f\left(\boldsymbol{\Sigma}_{X}^{t}\right)\right)$ for any $f \in B\left(\mathbb{R}^{N}\right)$. Let $\mathfrak{r}: \mathbb{R}^{N} \rightarrow \mathbb{R}^{N}$ be the reflection $\mathfrak{r}(s)=-s$ and $\bar{P}_{X}^{t}=P_{X}^{t} \circ \mathfrak{r}$. 
Proposition 4.2 If Assumptions (T1) (T2) hold, then for any $t \in \mathbb{R}$ the measures $P_{X}^{t}$ and $\bar{P}_{X}^{t}$ are equivalent and

$$
\frac{\mathrm{d} \bar{P}_{X}^{t}}{\mathrm{~d} P_{X}^{t}}(s)=\mathrm{e}^{-t X \cdot s}
$$

The proof of this proposition is very similar to the proof of Proposition 3.3 and we will omit it.

We shall call the universal relation (4.27) the finite time generalized Evans-Searles (GES) identity. As for the finite time ES identity, one can reformulate (4.27) in terms of the Laplace transform of $P_{X}^{t}$. To this end, consider the functional

$$
g_{t}(X, Y)=\log \omega_{X}\left(\mathrm{e}^{-Y \cdot \int_{0}^{t} \boldsymbol{\Phi}_{X s} \mathrm{~d} s}\right)=\log \int \mathrm{e}^{-t Y \cdot s} \mathrm{~d} P_{X}^{t}(s) .
$$

One easily sees that it inherits many properties of the Rényi entropy $e_{t}(\alpha)$. For fixed $X$ it is a convex function of $Y \in \mathbb{R}^{N}$ which satisfies $g_{t}(X, 0)=g_{t}(X, X)=0$. The lower bounds

$$
g_{t}(X, Y) \geq\left\{\begin{array}{l}
-t Y \cdot \omega_{X}\left(\boldsymbol{\Sigma}_{X}^{t}\right), \\
-t(X-Y) \cdot \omega_{X}\left(\boldsymbol{\Sigma}_{X}^{t}\right),
\end{array}\right.
$$

hold, and in particular $g_{t}(X, Y)>-\infty$. Most importantly, Proposition 4.2 is equivalent to the finite time GESsymmetry expressed by the following proposition.

Proposition 4.3 Under Assumptions (T1) (T2) one has

$$
g_{t}(X, Y)=g_{t}(X, X-Y)
$$

for any $X, Y \in \mathbb{R}^{N}$ and any $t \in \mathbb{R}$.

We again omit the proof which follows the same lines as the proof of Proposition 3.6 .

For later applications we recall the following elementary result (explicit in [LS2] and implicit in [Ga1]) which we shall call the symmetry lemma. We say that a function $a(X, Y)$ is $C^{1,2}$ in an open set $O \subset \mathbb{R}^{N} \times \mathbb{R}^{N}$ if all the partial derivatives $\partial_{X_{i}} a, \partial_{Y_{i}} a, \partial_{Y_{i}} \partial_{Y_{j}} a, \partial_{X_{j}} \partial_{Y_{i}} a$ and $\partial_{Y_{i}} \partial_{X_{j}} a$ exist and are continuous in $O$.

Lemma 4.4 Let the function $a(X, Y)$ be $C^{1,2}$ in a neighborhood of $(0,0) \in \mathbb{R}^{N} \times \mathbb{R}^{N}$ and such that

$$
a(X, Y)=a(X, X-Y) .
$$

Then

$$
\left.\partial_{X_{k}} \partial_{Y_{j}} a(X, Y)\right|_{X=Y=0}=-\left.\frac{1}{2} \partial_{Y_{j}} \partial_{Y_{k}} a(X, Y)\right|_{X=Y=0}
$$

Proof. The identity

$$
\left.\partial_{X_{k}} a(X, Y)\right|_{X=0}=\left.\partial_{X_{k}} a(X, X-Y)\right|_{X=0}=\left(\partial_{X_{k}} a\right)(0,-Y)+\left(\partial_{Y_{k}} a\right)(0,-Y),
$$

leads to

$$
\left.\partial_{Y_{j}} \partial_{X_{k}} a(X, Y)\right|_{X=Y=0}=-\left.\partial_{Y_{j}} \partial_{X_{k}} a(X, Y)\right|_{X=Y=0}-\left.\partial_{Y_{j}} \partial_{Y_{k}} a(X, Y)\right|_{X=Y=0} .
$$

The equality of mixed partial derivatives $\partial_{Y_{j}} \partial_{X_{k}} a=\partial_{X_{k}} \partial_{Y_{j}} a$ implies the statement. 


\subsection{Finite time linear response theory}

For any real or vector valued observable $f$ we set

$$
\langle f\rangle_{t}=\frac{1}{t} \int_{0}^{t} \omega\left(f_{s}\right) \mathrm{d} s .
$$

Finite time linear response theory is concerned with the first order perturbation theory w.r.t. $X$ of $\left\langle\boldsymbol{\Phi}_{X}\right\rangle_{t}$. Hence, in addition to (T1) (T2) we assume:

(T3) The function $X \mapsto\left\langle\Phi_{X}\right\rangle_{t}$ is differentiable at $X=0$ for all $t$.

The finite time kinetic transport coefficients are defined by

$$
L_{j k t}=\left.\partial_{X_{k}}\left\langle\Phi_{X}^{(j)}\right\rangle_{t}\right|_{X=0} .
$$

Since

$$
\left\langle\sigma_{X}\right\rangle_{t}=X \cdot\left\langle\boldsymbol{\Phi}_{X}\right\rangle_{t}=\sum_{j, k} X_{j} X_{k} L_{j k t}+o\left(|X|^{2}\right) \geq 0
$$

the real quadratic form determined by $\left[L_{j k t}\right]$ is positive semi-definite. This fact does not depend on the TRI assumption (T2) and does not imply that $L_{j k t}=L_{k j t}$. We shall call the relations

$$
L_{j k t}=L_{k j t},
$$

the finite time Onsager reciprocity relations (ORR). As general structural relations, they can hold only for TRI systems.

Another immediate consequence of Equ. (4.30) is:

Proposition 4.5 Suppose that (T1) holds and let $\mathbf{\Phi}_{X}, \widetilde{\mathbf{\Phi}}_{X}$ be two flux relations satisfying (T3), Then the corresponding finite time transport coefficients satisfy

$$
L_{j k t}+L_{k j t}=\widetilde{L}_{j k t}+\widetilde{L}_{k j t}
$$

If the finite time ORR hold, then $L_{j k t}=\widetilde{L}_{j k t}$.

In the next proposition we shall show that the finite time ORR follow from the finite time GES-symmetry establishing along the way the finite time Green-Kubo formula.

Proposition 4.6 Suppose that (T1) (T2) hold and that the function $g_{t}(X, Y)$ is $C^{1,2}$ in a neighborhood of $(0,0)$. Then (T3) holds and:

(1) The finite time Green-Kubo formula holds,

$$
L_{j k t}=\frac{1}{2} \int_{-t}^{t} \omega\left(\Phi^{(k)} \Phi_{s}^{(j)}\right)\left(1-\frac{|s|}{t}\right) \mathrm{d} s .
$$

(2) The finite time Onsager reciprocity relations hold,

$$
L_{j k t}=L_{k j t} .
$$


Proof. From the definition (4.28) we derive $\left.\partial_{Y_{j}} g_{t}(X, Y)\right|_{Y=0}=-t\left\langle\Phi_{X}^{(j)}\right\rangle_{t}$, and hence

$$
L_{j k t}=\left.\partial_{X_{k}}\left\langle\Phi_{X}^{(j)}\right\rangle_{t}\right|_{X=0}=-\left.\frac{1}{t} \partial_{X_{k}} \partial_{Y_{j}} g_{t}(X, Y)\right|_{X=Y=0} .
$$

The finite time GES-symmetry and the symmetry lemma yield

$$
L_{j k t}=\left.\frac{1}{2 t} \partial_{Y_{j}} \partial_{Y_{k}} g_{t}(X, Y)\right|_{X=Y=0}=\frac{1}{2 t} \int_{0}^{t} \int_{0}^{t} \omega\left(\Phi_{s_{1}}^{(k)} \Phi_{s_{2}}^{(j)}\right) \mathrm{d} s_{1} \mathrm{~d} s_{2} .
$$

Assertion (2) follows from the equality of mixed derivatives $\partial_{Y_{j}} \partial_{Y_{k}} g_{t}=\partial_{Y_{k}} \partial_{Y_{j}} g_{t}$. Since $\omega$ is invariant, we further get

$$
L_{j k t}=\frac{1}{2 t} \int_{0}^{t} \int_{0}^{t} \omega\left(\Phi^{(k)} \Phi_{s_{2}-s_{1}}^{(j)}\right) \mathrm{d} s_{1} \mathrm{~d} s_{2}=\frac{1}{2} \int_{-t}^{t} \omega\left(\Phi^{(k)} \Phi_{s}^{(j)}\right)\left(1-\frac{|s|}{t}\right) \mathrm{d} s,
$$

which proves Assertion (1). $\square$

We finish this section with two remarks.

Remark 1. The identity $\left(\Phi^{(k)} \Phi_{s}^{(j)}\right) \circ \vartheta=\Phi^{(k)} \Phi_{-s}^{(j)}$ implies that

$$
L_{j k t}=\int_{0}^{t} \omega\left(\Phi^{(k)} \Phi_{s}^{(j)}\right)\left(1-\frac{s}{t}\right) \mathrm{d} s .
$$

Remark 2. The covariance matrix $\mathbf{D}_{t}=\left[D_{j k t}\right]$ of the vector-valued random variable

$$
\frac{1}{\sqrt{t}} \int_{0}^{t} \boldsymbol{\Phi}_{s} \mathrm{~d} s
$$

with respect to $\omega$ is

$$
D_{j k t}=\int_{-t}^{t} \omega\left(\Phi^{(k)} \Phi_{s}^{(j)}\right)\left(1-\frac{|s|}{t}\right) \mathrm{d} s .
$$

The time-reversal plays no role in (4.33). However, if the assumptions of Proposition 4.6 hold and $\mathbf{L}_{t}=\left[L_{j k t}\right]$, then obviously

$$
\mathbf{D}_{t}=2 \mathbf{L}_{t} .
$$

These are the finite time Einstein relations which link the finite time covariance of fluxes in equilibrium to the finite time kinetic transport coefficients. Together with Proposition 4.6 they constitute the finite time FluctuationDissipation Theorem. We shall return to this topic at the end of Section 5.2

\subsection{Example: Thermally driven open systems}

We consider a system $S$, with phase space $M_{S}=\mathbb{R}^{n_{S}} \oplus \mathbb{R}^{n_{S}}$ and Hamiltonian $H_{S}\left(p_{S}, q_{S}\right)$, coupled to $N$ heat reservoirs $R_{1}, \ldots, R_{N}$. The phase space and the Hamiltonian of the $j$-th reservoir are $M_{j}=\mathbb{R}^{n_{j}} \oplus \mathbb{R}^{n_{j}}$ and $H_{j}\left(p_{j}, q_{j}\right)$. The phase space and the Hamiltonian of the composite system are

$$
M=M_{S} \oplus M_{1} \oplus \cdots \oplus M_{N}, \quad H_{0}(p, q)=H_{S}\left(p_{S}, q_{S}\right)+H_{1}\left(p_{1}, q_{1}\right)+\cdots+H_{N}\left(p_{N}, q_{N}\right),
$$

and we denote by $m$ the Lebesgue measure on $M$. 
The coupling between the system $S$ and the $j$-th reservoir is described by the Hamiltonian $V_{j}\left(p_{S}, p_{j}, q_{S}, q_{j}\right)$. The full Hamiltonian is

$$
H(p, q)=H_{0}(p, q)+V(p, q)=H_{0}(p, q)+\sum_{j=1}^{N} V_{j}\left(p_{S}, p_{j}, q_{S}, q_{j}\right) .
$$

We assume that $H$ is $C^{2}$ and that the finite energy subsets $\{(p, q) \mid H(q, p) \leq E\}$ are compact. These assumptions ensure that $H$ generates a global Hamiltonian flow $\phi^{t}$ of class $C^{1}$ on $M$. For any $C^{1}$ observable $F$,

$$
\frac{\mathrm{d} F_{t}}{\mathrm{~d} t}=\{H, F\}_{t},
$$

where $\{\cdot, \cdot\}$ denotes the Poisson bracket, $\{F, G\}=\nabla_{q} G \cdot \nabla_{p} F-\nabla_{p} G \cdot \nabla_{q} F$.

The state of the combined system in which each reservoir is at thermal equilibrium at inverse temperature $\beta_{j}$ and the system $S$ at inverse temperature $\beta$ is the product measure

$$
\frac{1}{Z} \mathrm{e}^{-\beta H_{S}-\sum_{j=1}^{N} \beta_{j} H_{j}} m
$$

Introducing the control parameters $X_{j}=\beta-\beta_{j}$, we can rewrite it as

$$
\omega_{X}=\frac{1}{Z} \mathrm{e}^{-\beta H_{0}+\sum_{j=1}^{N} X_{j} H_{j}} m .
$$

The dynamics does not depend on $X$ and we set $\phi_{X}^{t}=\phi^{t}$. Note that $\omega_{0}$ is not invariant under the flow $\phi^{t}$. In order to satisfy hypothesis (T1) one modifies (4.34) as

$$
\omega_{X}=\frac{1}{Z} \mathrm{e}^{-\beta\left(H_{S}+V\right)-\sum_{j=1}^{N} \beta_{j} H_{j}} m=\frac{1}{Z} \mathrm{e}^{-\beta H+\sum_{j=1}^{N} X_{j} H_{j}} m .
$$

With this definition, $\omega_{0}$ is the Gibbs canonical ensemble at inverse temperature $\beta$ and is invariant under $\phi^{t}$. Moreover, if the reservoirs have a large spatial extension and the coupling Hamiltonians $V_{j}$ are well localized, the states (4.34) and (4.35) describe the same thermodynamics.

For the reference state 4.35 the entropy cocycle is given by

$$
c_{X}^{t}=-\sum_{j=1}^{N} X_{j}\left(H_{j t}-H_{j}\right)=-\sum_{j=1}^{N} X_{j} \int_{0}^{t} \frac{\mathrm{d}}{\mathrm{d} s} H_{j s} \mathrm{~d} s,
$$

and we have the flux relation

$$
\sigma_{X}=-\sum_{j=1}^{N} X_{j}\left\{H, H_{j}\right\}=\sum_{j=1}^{N} X_{j}\left\{H_{j}, V\right\} .
$$

The flux observables do not depend on $X$ and are given by $\Phi^{(j)}=\left\{H_{j}, V\right\}$. Since

$$
H_{j t}-H_{j}=-\int_{0}^{t} \Phi_{s}^{(j)} \mathrm{d} s
$$

the flux observable $\Phi^{(j)}$ describes the flow of energy out of the $j$-th reservoir. The time reversal in physical systems is usually given by the map $\vartheta(p, q)=(-p, q)$ and the system $\left(M, \phi_{X}, \omega_{X}\right)$ is then TRI provided $H \circ \vartheta=H$.

We shall investigate a simple example of thermally driven open system in the remaining part of this section, to be continued in Sections 5.5.2 and 6.4.2. 


\subsubsection{The harmonic chain}

Quadratic Hamiltonians provide instructive examples of open systems whose non-equilibrium characteristics can be computed in a closed from [LS1]. From the mathematical point of view they are special cases of the Gaussian dynamical systems discussed in Section 9 Since the entropic fluctuations of such models are studied in detail in the forthcoming paper [JLTP], for reasons of space we shall be brief.

For a finite subset $\Gamma=\{n, n+1, \ldots, n+k-1\} \subset \mathbb{Z}$ we set $M^{\Gamma}=\mathbb{R}^{\Gamma} \oplus \mathbb{R}^{\Gamma}$ and we define

$$
M^{\Gamma} \ni(p, q) \mapsto H^{\Gamma}(p, q)=\sum_{x \in \mathbb{Z}} \frac{p_{x}^{2}+q_{x}^{2}}{2}+\frac{\left(q_{x}-q_{x-1}\right)^{2}}{2},
$$

where we set $p_{x}=q_{x}=0$ for $x \notin \Gamma$ (Dirichlet boundary conditions).

For some integer $m>0$, let $\Gamma_{S}=\{-m, \ldots, m\}$ and set $H_{S}=H^{\Gamma_{S}}$. This Hamiltonian describes a finite harmonic chain. We shall couple it to two large heat reservoirs, $R_{L}$ and $R_{R}$, at its two ends. For this purpose, let $n \gg m$ and set $\Gamma_{L}=\{-n, \ldots,-m-1\}, \Gamma_{R}=\{m+1, \ldots, n\}$. The Hamiltonians of the two reservoirs are

$$
H_{L}=H^{\Gamma_{L}}, \quad H_{R}=H^{\Gamma_{R}} .
$$

The Hamiltonian of the composite (but still decoupled) system is

$$
H_{0}=H^{\Gamma_{L}}+H^{\Gamma_{S}}+H^{\Gamma_{R}}=H_{L}+H_{S}+H_{R} .
$$

Finally, define the Hamiltonian of the coupled system by

$$
H=H^{\Gamma_{L} \cup \Gamma_{S} \cup \Gamma_{R}} .
$$

The coupling Hamiltonian is given by

$$
V=H-H_{0}=V_{L}+V_{R}=-q_{-m-1} q_{-m}-q_{m} q_{m+1},
$$

and is independent of $n$. Since the equations of motion induced by $H_{0}$ and $H$ are linear, the associated Hamiltonian flows are linear group which we write as $\mathrm{e}^{t \mathcal{L}_{0}}$ and $\mathrm{e}^{t \mathcal{L}}$ respectively.

Let us denote by $h, h_{L}, h_{R}$ the real symmetric matrices corresponding to the quadratic forms $2 H, 2 H_{L}, 2 H_{R}$. The reference state $\omega_{X}$ is the centered Gaussian measure of covariance

$$
D_{X}=(\beta h-k(X))^{-1},
$$

where

$$
k(X)=X_{L} h_{L} \oplus X_{R} h_{R} .
$$

For $\beta>0$ the set $O_{\beta}=\left\{X \in \mathbb{R}^{2} \mid \beta h-k(X)>0\right\}$ is an open neighborhood of 0 . The dynamical system thus obtained is well defined for $X \in O_{\beta}$, is TRI and clearly satisfies Assumptions (T1) and (T2)

It is a simple exercise in Gaussian integrals to show that Assumption (E1) is satisfied for $X$ small enough. However, note that the flux observables

$$
\begin{aligned}
& \Phi^{(L)}=\left\{H_{L}, V\right\}=-p_{-m-1} q_{-m}, \\
& \Phi^{(R)}=\left\{H_{R}, V\right\}=-p_{m+1} q_{m},
\end{aligned}
$$

as well as entropy production $\sigma_{X}=X_{L} \Phi^{(L)}+X_{R} \Phi^{(R)}$ are unbounded. Thus, Hypothesis (E2) is not satisfied. 
Propositions 4.2 and 4.3 apply. Moreover, the functional $g_{t}(X, Y)$ reduces to a Gaussian integral that can be computed explicitly

$$
g_{t}(X, Y)=-\frac{1}{2} \log \operatorname{det}\left(I-D_{X}\left(\mathrm{e}^{t \mathcal{L}^{*}} k(Y) \mathrm{e}^{t \mathcal{L}}-k(Y)\right)\right),
$$

with the convention that $\log x=-\infty$ for $x \leq 0$. Since the groups $\mathrm{e}^{t \mathcal{L}}$ is uniformly bounded, the function $(X, Y) \mapsto g_{t}(X, Y)$ is real analytic on an open neighborhood of $(0,0)$ in $O_{\beta} \times \mathbb{R}^{2}$ which is independent of $t \in \mathbb{R}$ and Proposition 4.6 applies too.

The validity of the finite time GES-symmetry, ensured by Proposition 4.3, can be explicitly checked as follows. Energy conservation, $\mathrm{e}^{t \mathcal{L}^{*}} h \mathrm{e}^{t \mathcal{L}}=h$, yields

$$
\begin{aligned}
D_{X}^{-1}-\left(\mathrm{e}^{t \mathcal{L}^{*}} k(Y) \mathrm{e}^{t \mathcal{L}}-k(Y)\right) & =\beta h-k(X-Y)-\mathrm{e}^{t \mathcal{L}^{*}} k(Y) \mathrm{e}^{t \mathcal{L}} \\
& =\mathrm{e}^{t \mathcal{L}^{*}}\left(\beta h-k(Y)-\mathrm{e}^{-t \mathcal{L}^{*}} k(X-Y) \mathrm{e}^{-t \mathcal{L}}\right) \mathrm{e}^{t \mathcal{L}} \\
& =\mathrm{e}^{t \mathcal{L}^{*}}\left(D_{X}^{-1}-\left(\mathrm{e}^{-t \mathcal{L}^{*}} k(X-Y) \mathrm{e}^{-t \mathcal{L}}-k(X-Y)\right)\right) \mathrm{e}^{t \mathcal{L}} .
\end{aligned}
$$

This relation and Liouville's theorem, $\operatorname{det} \mathrm{e}^{t \mathcal{L}}=\operatorname{det} \mathrm{e}^{t \mathcal{L}^{*}}=1$, imply that $g_{t}(X, Y)=g_{-t}(X, X-Y)$. Finally, TRI yields $g_{-t}(X, X-Y)=g_{t}(X, X-Y)$ and the finite time GES-symmetry follows.

\section{The large time limit}

\subsection{Entropy production}

For any observable $f$ we set

$$
\langle f\rangle_{+}=\lim _{t \rightarrow \infty}\langle f\rangle_{t}
$$

whenever this limit exists.

In this section in addition to (E1) (E2) we assume:

(E3) The limit $\langle\sigma\rangle_{+}$exists and is finite.

The entropy balance equation yields the basic result:

Proposition $5.1\langle\sigma\rangle_{+} \geq 0$.

We shall say that the dynamical system $(M, \phi, \omega)$ is entropy producing if it satisfies

$$
\text { (EP) }\langle\sigma\rangle_{+}>0 \text {. }
$$

The validity of (E3) and (EP) are dynamical problems that can only be answered in the context of concrete models. In this section we shall discuss several structural results which shed some light on these central issues.

\section{Proposition 5.2 Suppose that}

$$
\langle\sigma\rangle_{t}=\langle\sigma\rangle_{+}+O\left(t^{-1}\right)
$$

as $t \rightarrow \infty$. Then $\langle\sigma\rangle_{+}=0$ implies that there exists $\nu \in \mathcal{S}_{I} \cap \mathcal{N}_{\omega}$ satisfying $\operatorname{Ent}(\nu \mid \omega)>-\infty$. 
Proof: We shall use the properties of relative entropy listed in Theorem 2.1 Suppose that $\langle\sigma\rangle_{+}=0$. The entropy balance equation and (5.36) yield

$$
\left|\operatorname{Ent}\left(\omega_{t} \mid \omega\right)\right|=\left|\int_{0}^{t}\left(\omega\left(\sigma_{s}\right)-\langle\sigma\rangle_{+}\right) \mathrm{d} s\right|=O(1) .
$$

Hence, there is $C$ such that $\operatorname{Ent}\left(\omega_{t} \mid \omega\right) \geq C$ for all $t \geq 0$. Set $\tilde{\omega}_{t}(\cdot)=t^{-1} \int_{0}^{t} \omega_{s}(\cdot) \mathrm{d} s$. The concavity and the upper-semicontinuity of the relative entropy yield $\operatorname{Ent}\left(\tilde{\omega}_{t} \mid \omega\right) \geq C$. By compactness, there exists $\nu \in \mathcal{S}$ and a net $t_{\alpha} \rightarrow \infty$ such that $\tilde{\omega}_{t_{\alpha}} \rightarrow \nu$. It follows easily that $\nu \in \mathcal{S}_{I}$ and the upper-semicontinuity implies $\operatorname{Ent}(\nu \mid \omega)>-\infty$.

Proposition 5.3 Let $\nu \in \mathcal{S}_{I} \cap \mathcal{N}_{\omega}$. Then $\nu(\sigma)=0$.

Before proving Proposition 5.3, we need a preliminary result which is of independent interest. In what follows we shall say that a sequence $t_{n} \uparrow \infty$ is regular if $\sum_{n} \mathrm{e}^{-a t_{n}}<\infty$ for all $a>0$.

Lemma 5.4 Let $t_{n}$ be a regular sequence. Then, for $\omega$-a.e. $x$,

$$
\liminf _{n \rightarrow \infty} \frac{1}{t_{n}} \int_{0}^{t_{n}} \sigma_{s}(x) \mathrm{d} s \geq 0, \quad \limsup _{n \rightarrow \infty} \frac{1}{t_{n}} \int_{0}^{t_{n}} \sigma_{-s}(x) \mathrm{d} s \leq 0 .
$$

Proof. We will prove the first relation in 5.37), a similar argument yields the second.

Let $X_{n}=t_{n}^{-1} \int_{0}^{t_{n}} \sigma_{s} \mathrm{~d} s$ and $A=\left\{x \in M \mid \liminf _{n \rightarrow \infty} X_{n}(x)<0\right\}$. We need to show that $\omega(A)=0$. Since $A=\cup_{k \geq 1} A_{k}$ with $A_{k}=\left\{x \in M \mid \liminf _{n \rightarrow \infty} X_{n}(x) \leq-1 / k\right\}$, it suffices to show that $\omega\left(A_{k}\right)=0$ for all integers $k \geq 1$. Set $\Delta^{t}=\Delta_{\omega_{t} \mid \omega}$ and note that $\omega\left(\Delta^{-t}\right)=\omega_{-t}(1)=1$, for all $t$. The Markov inequality gives

$$
\omega\left(\left\{x \in M \mid \Delta^{-t}(x) \geq \lambda\right\}\right) \leq \lambda^{-1},
$$

for $\lambda>0$. Since $\Delta^{-t_{n}}=\mathrm{e}^{-t_{n} X_{n}}$, we have

$$
\omega\left(\left\{x \in M \mid X_{n}(x) \leq-a\right\}\right)=\omega\left(\left\{x \in M \mid \Delta^{-t_{n}}(x) \geq \mathrm{e}^{a t_{n}}\right\}\right) \leq \mathrm{e}^{-a t_{n}} .
$$

Hence,

$$
\sum_{n} \omega\left(\left\{x \in M \mid X_{n}(x) \leq 1 / k\right\}\right)<\infty,
$$

and the Borel-Cantelli lemma yields that $\omega\left(A_{k}\right)=0$.

Proof of Proposition 5.3. By the ergodic theorem, there is $\bar{\sigma} \in L^{1}(M, \mathrm{~d} \nu)$ such that

$$
\lim _{n \rightarrow \infty} \frac{1}{n} \int_{0}^{n} \sigma_{ \pm s}(x) \mathrm{d} s=\bar{\sigma}(x),
$$

for $\nu$-a.e. $x$. Since $\nu$ is invariant one has $\nu(\sigma)=\nu(\bar{\sigma})$. Since $\nu$ is normal w.r.t. $\omega$, Lemma 5.4 implies that $\nu(\bar{\sigma})=0$ and the statement follows.

Corollary 5.5 Suppose that $\mathcal{S}_{I} \cap \mathcal{N}_{\omega} \neq \emptyset$ and that there exists a sequence $t_{n} \uparrow \infty$ such that

$$
\lim _{n \rightarrow \infty} \frac{1}{t_{n}} \int_{0}^{t_{n}} \sigma_{s}(x) \mathrm{d} s=\langle\sigma\rangle_{+},
$$

for w-a.e. $x$. Then $\langle\sigma\rangle_{+}=0$. 
Proof. Let $\nu \in \mathcal{S}_{I} \cap \mathcal{N}_{\omega}$. Then

$$
\langle\sigma\rangle_{+}=\nu\left(\lim _{n \rightarrow \infty} \frac{1}{t_{n}} \int_{0}^{t_{n}} \sigma_{s}(x) \mathrm{d} s\right)=\nu(\sigma)=0 .
$$

The results of this section establish that, under very general conditions, the dynamical system $(M, \phi, \omega)$ is entropy producing iff $\mathcal{S}_{I} \cap \mathcal{N}_{\omega}=\emptyset$.

\subsection{Linear response theory}

Consider a family $\left(M, \phi_{X}, \omega_{X}, \Phi_{X}\right)$ satisfying (T1), (T2) and (T3) In this section we are interested in the large time limit of the time averaged expectation values of individual fluxes and validity of the linear response theory. In addition to (T1)-(T3) we assume:

(T4) The limit $\left\langle\boldsymbol{\Phi}_{X}\right\rangle_{+}$exists for $X$ small enough and is differentiable at $X=0$.

$$
\text { (T5) } \omega\left(\Phi^{(k)} \Phi_{t}^{(j)}\right)=O\left(t^{-1}\right) \text { as } t \rightarrow \infty \text {. }
$$

The verification of (T4) and (T5) is a dynamical problem that can be answered only in the context of concrete models. (T2) and (T5) imply that $\omega\left(\Phi^{(k)} \Phi_{t}^{(j)}\right)=O\left(t^{-1}\right)$ as $t \rightarrow-\infty$.

The kinetic transport coefficients are defined by

$$
L_{j k}=\left.\partial_{X_{k}}\left\langle\Phi_{X}^{(j)}\right\rangle_{+}\right|_{X=0} .
$$

Since $\left\langle\sigma_{X}\right\rangle_{+}=\sum_{j} X_{j}\left\langle\Phi_{X}^{(j)}\right\rangle_{+} \geq 0$, the real quadratic form determined by $\left[L_{j k}\right]$ is positive semi-definite.

The kinetic transport coefficients satisfy the Onsager reciprocity relations (ORR) if

$$
L_{j k}=L_{k j}
$$

and the Green-Kubo formula holds if

$$
L_{j k}=\frac{1}{2} \int_{-\infty}^{\infty} \omega\left(\Phi^{(k)} \Phi_{s}^{(j)}\right) \mathrm{d} s
$$

where, unless otherwise specified, $\int_{-\infty}^{\infty}=\lim _{t \rightarrow \infty} \int_{-t}^{t}$. Note that (5.40) $\Rightarrow 5.39$.

The finite time linear response theory leads to a natural axiomatic program for the verification of (5.39) and (5.40) based on the following

Proposition 5.6 Suppose that (T1)-(T5) hold. Then the following statements are equivalent:

(1) The Green-Kubo formulas (5.40) hold.

(2) $\lim _{t \rightarrow \infty} L_{j k t}=L_{j k}$. 
Proof. Set

$$
F(t)=\frac{1}{2} \int_{-t}^{t} \omega\left(\Phi^{(k)} \Phi_{s}^{(j)}\right) \mathrm{d} s
$$

and notice that

$$
L_{j k t}=\frac{1}{2} \int_{-t}^{t} \omega\left(\Phi^{(k)} \Phi_{s}^{(j)}\right)\left(1-\frac{|s|}{t}\right) \mathrm{d} s=\frac{1}{t} \int_{0}^{t} F(s) \mathrm{d} s .
$$

By the fundamental property of Cesàro's mean, (1) $\Rightarrow \lim _{t \rightarrow \infty} F(t)=L_{j k} \Rightarrow$ (2). On the other hand, Hypothesis (T5) and Hardy-Littlewood's Tauberian theorem (see e.g. [Ko] ) yield (2) $\Rightarrow \lim _{t \rightarrow \infty} F(t)=L_{j k} \Rightarrow(1)$.

We would like to add several remarks regarding Proposition 5.6

Remark 1. Finite time linear response theory requires the minimal regularity assumptions (T1) [(T3), and in particular no ergodicity assumption. It is valid in practically all models of interest. Assumption (T4) states that the basic objects of linear response theory are well-defined (existence of the $L_{j k}$ 's) and is of course necessary to have a meaningful theory. Condition (2) of Proposition 5.6 can be reformulated as

$$
\left.\partial_{X_{k}}\left(\lim _{t \rightarrow \infty}\left\langle\Phi_{X}^{(j)}\right\rangle_{t}\right)\right|_{X=0}=\lim _{t \rightarrow \infty}\left(\left.\partial_{X_{k}}\left\langle\Phi_{X}^{(j)}\right\rangle_{t}\right|_{X=0}\right)
$$

i.e., as an exchange of the two limits $t \rightarrow \infty$ and $X \rightarrow 0$. Even though the existence of the improper integral in (5.40) does not require any decay of the correlation function $t \mapsto \omega\left(\Phi^{(k)} \Phi_{t}^{(j)}\right)$, Assumption (T5) provides the minimal decay assumption which ensures that

$$
\lim _{t \rightarrow \infty} L_{j k t}=\lim _{t \rightarrow \infty} \frac{1}{2} \int_{-t}^{t} \omega\left(\Phi^{(k)} \Phi_{s}^{(j)}\right) \mathrm{d} s .
$$

Note however that Assumption (T5) is needed only for the Green-Kubo formula and that Condition (2) automatically implies ORR. Assumptions (T4) and (T5)] are ergodic in nature and are typically difficult to verify in physically interesting models. A particularly delicate aspect is differentiability of the function $X \mapsto\left\langle\boldsymbol{\Phi}_{X}\right\rangle_{+}$.

Remark 2. The proposed program for the derivation of linear response theory is in a certain sense minimal. On physical grounds one would like to have an additional estimate

$$
\left\langle\boldsymbol{\Phi}_{X}\right\rangle_{t}=\mathbf{L} X+\operatorname{Er}(X, t),
$$

where the error term satisfies

$$
\lim _{X \rightarrow 0} \sup _{t>0} \frac{|\operatorname{Er}(X, t)|}{|X|}=0,
$$

with the rate of convergence/range of parameters that allow to draw physical/numerical conclusion from (5.42). This point is related to van Kampen's objections against linear response theory [Ka, KTH], see [CELS2] for a discussion.

Remark 3. In some models the following well-known result (the multivariable Vitali theorem) can be effectively used to verify (5.41) (see [JPP]). Let $I_{\epsilon}=\left\{X \in \mathbb{R}^{N}|| X \mid<\epsilon\right\}$ and $D_{\epsilon}=\left\{X \in \mathbb{C}^{N}|| X \mid<\epsilon\right\}$.

Proposition 5.7 For all $t>0$ let $F_{t}: D_{\epsilon} \rightarrow \mathbb{C}$ be an analytic function such that

$$
\sup _{X \in D_{\epsilon}, t>0}\left|F_{t}(X)\right|<\infty
$$

and assume that

$$
\lim _{t \rightarrow \infty} F_{t}(X)=F(X)
$$

exists for $X \in I_{\epsilon}$. Then the limit (5.43) exist for all $X \in D_{\epsilon}$ and is an analytic function on $D_{\epsilon}$. Moreover, as $t \rightarrow \infty$, all derivatives of $F_{t}$ converge uniformly on compact subsets of $D_{\epsilon}$ to the corresponding derivatives of $F$. 
We shall point out some mathematical intricacies regarding the interchange of the limit and derivative in (5.41) on a simple example in Section 8.3

Our final topic in this section is the Fluctuation-Dissipation Theorem (recall its finite time counterpart discussed in Remark 2 of Section 4.3).

Definition 5.8 Suppose that (T1)-(T4) hold. We shall say that the Fluctuation-Dissipation Theorem holds for $\left.\left(M, \phi_{X}, \omega_{X}, \boldsymbol{\Phi}_{X}\right)\right)$ if:

(1) The Green-Kubo formulas (and hence the Onsager reciprocity relations) hold for the kinetic transport coeffcients $\mathbf{L}=\left[L_{j k}\right]$.

(2) The Central Limit Theorem holds for $\boldsymbol{\Phi}=\left(\Phi^{(1)}, \cdots, \Phi^{(N)}\right)$ w.r.t. $(M, \phi, \omega)$ with covariance matrix

$$
\mathbf{D}=2 \mathbf{L},
$$

i.e., for any Borel set $B \subset \mathbb{R}^{N}$,

$$
\lim _{t \rightarrow \infty} \omega\left(\left\{x \in M \mid \frac{1}{\sqrt{t}} \int_{0}^{t} \mathbf{\Phi}_{s} \mathrm{~d} s \in B\right\}\right)=\mu_{\mathbf{D}}(B)
$$

where $\mu_{\mathbf{D}}$ is the centered Gaussian measure of covariance $\mathbf{D}$ on $\mathbb{R}^{n}$.

Remark. The celebrated Einstein's relations (5.44) link equilibrium fluctuations to kinetic transport coefficients.

Just like Proposition 5.6 the Fluctuation-Dissipation Theorem is "forced" by its universally valid finite time counterpart. With regard to the proof of the Central Limit Theorem, we mention the following result of Bryc [Bry]. $D_{\epsilon}$ and $I_{\epsilon}$ are as in Proposition 5.7

Proposition 5.9 Assume that (T1) holds. Suppose that for some $\epsilon>0$ the function

$$
g_{t}(0, Y)=\log \omega\left(\mathrm{e}^{-Y \cdot \int_{0}^{t} \boldsymbol{\Phi}_{s} \mathrm{~d} s}\right),
$$

is analytic in $D_{\epsilon}$, satisfies

$$
\sup _{Y \in D_{\epsilon}, t>1} \frac{1}{t}\left|g_{t}(0, Y)\right|<\infty
$$

and that

$$
\lim _{t \rightarrow \infty} \frac{1}{t} g_{t}(0, Y),
$$

exists for all $Y \in I_{\epsilon}$. Then the Central Limit Theorem holds for $\boldsymbol{\Phi}=\left(\Phi^{(1)}, \cdots, \Phi^{(N)}\right)$ w.r.t. $(M, \phi, \omega)$ with covariance matrix

$$
D_{j k}=\lim _{t \rightarrow \infty} \int_{-t}^{t} \omega\left(\Phi^{(k)} \Phi_{s}^{(j)}\right)\left(1-\frac{|s|}{t}\right) \mathrm{d} s .
$$

Remark. If $\Phi^{(j)} \in L^{\infty}(M, \mathrm{~d} \omega)$, then the function $Y \mapsto g_{t}(0, Y)$ is real analytic. The location of the complex zeros of the entire analytic function $Y \mapsto \omega\left(\mathrm{e}^{-Y \cdot \int_{0}^{t} \boldsymbol{\Phi}_{s} \mathrm{~d} s}\right)$ determines the region of complex plane to which $g_{t}(0, Y)$ extends analytically. If in addition (T2), (T3) and (T5) hold, the existence of the limit (5.46) implies that

$$
\lim _{t \rightarrow \infty} L_{j k t}=\frac{1}{2} \int_{-\infty}^{\infty} \omega\left(\Phi^{(k)} \Phi_{s}^{(j)}\right) \mathrm{d} s=\frac{1}{2} D_{j k}
$$

The Fluctuation-Dissipation Theorem is the pillar of non-equilibrium statistical mechanics in the regime where the thermodynamic forces are weak. The far from equilibrium case is discussed in the next section. 


\subsection{The Evans-Searles Fluctuation Theorem}

We start by recalling some basic facts of the Large Deviation Theory (see e.g. [DZ, El]).

Definition 5.10 A vector-valued observable $\mathbf{f}=\left(f^{(1)}, \cdots, f^{(N)}\right)$ satisfies a Large Deviation Principle w.r.t. to $(M, \phi, \omega)$ if there exists an upper-semicontinuous function $I: \mathbb{R}^{N} \rightarrow[-\infty, 0]$ with compact level sets such that for all Borel sets $G \subset \mathbb{R}^{N}$ we have

$$
\begin{aligned}
\sup _{Z \in \mathscr{G}} I(Z) & \leq \liminf _{t \rightarrow \infty} \frac{1}{t} \log \omega\left(\left\{x \in M \mid \frac{1}{t} \int_{0}^{t} \mathbf{f}_{s}(x) \mathrm{d} s \in G\right\}\right) \\
& \leq \limsup _{t \rightarrow \infty} \frac{1}{t} \log \omega\left(\left\{x \in M \mid \frac{1}{t} \int_{0}^{t} \mathbf{f}_{s}(x) \mathrm{d} s \in G\right\}\right) \leq \sup _{Z \in \bar{G}} I(Z) .
\end{aligned}
$$

where $\stackrel{\circ}{G}$ denotes the interior of $G$ and $\bar{G}$ its closure.

The following standard result goes under the name of Gärtner-Ellis Theorem and will be used repeatedly, see e.g. [El, DZ] for a proof.

Proposition 5.11 Assume that the limit

$$
h(Y)=\lim _{t \rightarrow \infty} \frac{1}{t} \log \omega\left(e^{-\int_{0}^{t} Y \cdot \mathbf{f}_{\mathbf{s}} \mathrm{d} s}\right),
$$

exists in $[-\infty,+\infty]$ for all $Y \in \mathbb{R}^{N}$ and is finite for $Y$ in some open neighborhood of $0 \in \mathbb{R}^{N}$.

(1) Suppose that $h(Y)$ is differentiable at $Y=0$. Then, the limit

$$
\langle\mathbf{f}\rangle_{+}=\lim _{t \rightarrow \infty} \frac{1}{t} \int_{0}^{t} \omega\left(\mathbf{f}_{s}\right) \mathrm{d} s,
$$

exists and $\langle\mathbf{f}\rangle_{+}=-\nabla h(0)$. Moreover, for any regular sequence $t_{n}$ one has

$$
\lim _{n \rightarrow \infty} \frac{1}{t_{n}} \int_{0}^{t_{n}} \mathbf{f}_{s}(x) \mathrm{d} s=\langle\mathbf{f}\rangle_{+},
$$

for w-a.e. $x$.

(2) Suppose that $h(Y)$ is a lower semicontinuous function on $\mathbb{R}^{N}$ which is differentiable on the interior of the set $\mathcal{D}=\left\{Y \in \mathbb{R}^{N} \mid h(Y)<\infty\right\}$ and satisfies

$$
\lim _{\mathcal{D} \ni Y \rightarrow Y_{0}}|\nabla h(Y)|=\infty,
$$

for all $Y_{0} \in \partial \mathcal{D}$. Then the Large Deviation Principle holds for $\mathbf{f}$ w.r.t. $(M, \phi, \omega)$ with the rate function

$$
I(Z)=\inf _{Y \in \mathbb{R}^{N}}(Y \cdot Z+h(Y)),
$$

i.e., $-I(Z)$ is the Legendre transform of $h(-Y)$. In particular, $I(Z)$ is concave.

Remark 5.12 The conclusion of Part (2) holds in particular if $h(Y)$ is differentiable on $\mathbb{R}^{N}$. There are other (local) versions of Gärtner-Ellis theorems that are useful in applications. Suppose, for example, that the function $h(Y)$ is finite, strictly convex and continuously differentiable in some open neighborhood $B \subset \mathbb{R}^{N}$ of the origin. Then Part (1) holds and a weaker version of part (2) also holds: the large deviation bounds (5.47) hold provided the set $G$ is contained in a sufficiently small neighborhood of the mean $\langle\mathbf{f}\rangle_{+}$(see Lemma XIII.2 of [HH] and Section 4.5 of [DZ]). 
Let $(M, \phi, \omega)$ be a TRI system. Recall that

$$
e_{t}(\alpha)=\log \omega\left(\mathrm{e}^{-\alpha \int_{0}^{t} \sigma_{s} \mathrm{~d} s}\right)
$$

We suppose:

(ES) The Evans-Searles functional (ES-functional for short)

$$
\mathbb{R} \ni \alpha \mapsto e(\alpha)=\lim _{t \rightarrow \infty} \frac{1}{t} e_{t}(\alpha) \in[-\infty, \infty],
$$

exists.

Propositions 3.4 and 3.5 yield the basic properties of the ES-functional :

Proposition 5.13 (1) $e(\alpha)$ is a convex function of $\alpha$.

(2) It satisfies the ES-symmetry

$$
e(\alpha)=e(1-\alpha)
$$

(3)

$$
\begin{gathered}
e(0)=e(1)=0, \\
\begin{cases}e(\alpha) \leq 0 \quad \text { if } \alpha \in[0,1], \\
e(\alpha) \geq 0 \quad \text { otherwise }\end{cases}
\end{gathered}
$$

(4) It satisfies the lower bound

$$
e(\alpha) \geq\left(\left|\alpha-\frac{1}{2}\right|-\frac{1}{2}\right) \Sigma^{+}
$$

with

$$
\Sigma^{+}=\limsup _{t \rightarrow \infty} \omega\left(\Sigma^{t}\right) .
$$

In particular, if (E3) holds then $\Sigma^{+}=\langle\sigma\rangle_{+}$.

We emphasize that the ES-symmetry (5.48) is an immediate consequence of the finite time ES-symmetry.

Using Proposition 5.11 we obtain

Proposition 5.14 (1) Suppose that $e(\alpha)$ is differentiable at $\alpha=0$. Then (E3) holds and $\langle\sigma\rangle_{+}=-e^{\prime}(0)$.

(2) Suppose that $e(\alpha)$ is differentiable for all $\alpha \in \mathbb{R}$. Then the Large Deviation Principle hold for the entropy production observable $\sigma$ w.r.t. $(M, \phi, \omega)$ with the concave rate function $I(s)=\inf _{\alpha \in \mathbb{R}}(s \alpha+e(\alpha))$. Moreover

$$
I(s)=s+I(-s) .
$$

Proof. We only need to prove (5.50). Using (5.48) we have

$$
I(s)=\inf _{\alpha}(s \alpha+e(\alpha))=\inf _{\alpha}(s(1-\alpha)+e(1-\alpha))=s+\inf _{\alpha}(-s \alpha+e(\alpha))=s+I(-s) .
$$

The relation (5.50) is called the ES-symmetry for the rate function $I(s)$.

Consider now a family $\left(M, \phi_{X}, \omega_{X}, \mathbf{\Phi}_{X}\right)$ indexed by $X \in \mathbb{R}^{N}$ and satisfying Assumptions (T1), (T2) and (T3). We assume: 
(GES) The Generalized Evans-Searles functional (GES-functional)

$$
g(X, Y)=\lim _{t \rightarrow \infty} \frac{1}{t} g_{t}(X, Y)
$$

exists for all $X, Y$.

$g(X, Y)$ is a convex function of $Y$ and the finite time GES-symmetry implies that

$$
g(X, Y)=g(X, X-Y) .
$$

We shall refer to this relation as the GES-symmetry.

Proposition 5.15 (1) Suppose that $Y \mapsto g(X, Y)$ is differentiable at 0. Then (T4) holds,

$$
\left\langle\boldsymbol{\Phi}_{X}\right\rangle_{+}=-\left.\nabla_{Y} g(X, Y)\right|_{Y=0},
$$

and if $t_{n}$ is a regular sequence, then

$$
\lim _{n \rightarrow \infty} \frac{1}{t_{n}} \int_{0}^{t_{n}} \boldsymbol{\Phi}_{X s}(x) \mathrm{d} s=\left\langle\boldsymbol{\Phi}_{X}\right\rangle_{+},
$$

for $\omega_{X}$-a.e. $x$.

(2) Suppose that $Y \mapsto g(X, Y)$ is differentiable for all $Y$. Then the Large Deviation Principle holds for the flux observables w.r.t. $\left(M, \phi_{X}, \omega_{X}\right)$ with the concave rate function $I_{X}(s)=\inf _{Y \in \mathbb{R}^{N}}(Y \cdot s+g(X, Y))$. Moreover

$$
I_{X}(s)=X \cdot s+I_{X}(-s) .
$$

(3) Suppose that $g(X, Y)$ is $C^{1,2}$ in a neighborhood of $(0,0)$. Then the kinetic transport coefficients are defined and satisfy the Onsager reciprocity relations.

(4) In addition to the assumption of (3) suppose that (T5) holds and that for some $\epsilon>0$,

$$
\sup _{Y \in D_{\epsilon}, t>1} \frac{1}{t}\left|g_{t}(0, Y)\right|<\infty .
$$

Then the Fluctuation-Dissipation Theorem holds for $\left(M, \phi_{X}, \omega_{X}, \mathbf{\Phi}_{X}\right)$.

Proof. (1) and (2) are immediate from Proposition 5.11 and the symmetry of $I_{X}(s)$ is proved as in Proposition 5.14

(3) By Assertion (1), $\left\langle\Phi_{X}^{(j)}\right\rangle_{+}=\left.\partial_{Y_{j}} g(X, Y)\right|_{Y=0}$, hence the GES-symmetry and the symmetry lemma yield

$$
L_{j k}=\left.\partial_{X_{k}} \partial_{Y_{j}} g(X, Y)\right|_{X=Y=0}=-\left.\frac{1}{2} \partial_{Y_{j}} \partial_{Y_{k}} g(X, Y)\right|_{X=Y=0} .
$$

Since the partial derivatives on the right hand side are symmetric in $j, k$, we have $L_{j k}=L_{k j}$.

(4) From (5.52) and the fact that

$$
\left.\partial_{Y_{j}} \partial_{Y_{k}} \frac{1}{t} \log g_{t}(0, Y)\right|_{Y=0}=-\int_{-t}^{t} \omega\left(\Phi^{(k)} \Phi_{s}^{(j)}\right)\left(1-\frac{|s|}{t}\right) \mathrm{d} s
$$


we see that the Green-Kubo formula holds iff the limit and the derivative in the expression

$$
\left.\partial_{Y_{j}} \partial_{Y_{k}} g(0, Y)\right|_{Y=0}=\left.\partial_{Y_{j}} \partial_{Y_{k}} \lim _{t \rightarrow \infty} \frac{1}{t} g_{t}(0, Y)\right|_{Y=0},
$$

can be interchanged. This is ensured by the assumption (5.51) and Proposition 5.7 Similarly, Proposition 5.9 yields the CLT.

We shall say that a given TRI model satisfies the Evans-Searles Fluctuation Theorem if the respective functionals $e(\alpha) / g(X, Y)$ exist and are differentiable/ $C^{1,2}$. It follows from Propositions 5.14 and 5.15 that the the EvansSearles Fluctuation Theorem can be interpreted as an extension of the fluctuation-dissipation to the far from equilibrium region.

Remark 1. The sufficient and necessary condition for the validity of the Green-Kubo formula is that the limit and the derivative in the formula (5.53) can be interchanged. Assumption (5.51) provides a convenient criterion for validity of this exchange which will be satisfied in several examples that we will consider. In general, however, there may exist other mechanisms that will lead to the justification of (5.53), see for example the proof of the Fluctuation-Dissipation theorem for the Sinai billiard with small external forces in [CELS1, CELS2, Ch1, Ch2].

Remark 2. It is instructive to compare (3) and (4) to the finite time based derivation of the linear response theory presented in Section 5.2

Remark 3. In some models where the entropy production observable is unbounded the ES-functional $e_{t}(\alpha)$ is finite only on an open interval containing $[0,1]$. In this case one can still formulate a meaningful Evans-Searles Fluctuation Theorem, see Section 9 for an example. The same remark applies to the GES-functional (see Section 5.5.2).

\subsection{The resonance interpretation of $e(\alpha)$}

Under suitable regularity conditions, the identity

$$
e_{t}(\alpha)=\log \left(1, \mathrm{e}^{t L_{p}} 1\right),
$$

for $p=1 / \alpha$ leads to identification of $e(\alpha)$ with a resonance of $L^{p}$-Liouvillean $L_{p}$. In this section we state two general results regarding this identification.

Since $\operatorname{sp}\left(L_{p}\right) \subset\left\{z|| \operatorname{Re} z \mid \leq m_{p}\right\}$, the resolvent $\left(z-L_{p}\right)^{-1}$ is a well-defined operator valued function analytic in the half-plane $\operatorname{Re} z>m_{p}$. Note that $|e(\alpha)| \leq m_{p}$ and that

$$
\left(1,\left(z-L_{p}\right)^{-1} 1\right)=\int_{0}^{\infty} \mathrm{e}^{e_{t}\left(p^{-1}\right)-t z} \mathrm{~d} t,
$$

for $\operatorname{Re} z>m_{p}$.

In the next two propositions $\alpha \in \mathbb{R}$ is fixed and $p=1 / \alpha$.

Proposition 5.16 Suppose that for some $\gamma>0$ and $c \in \mathbb{R}$,

$$
e_{t}(\alpha)=t e(\alpha)+c+O\left(\mathrm{e}^{-\gamma t}\right),
$$

as $t \rightarrow \infty$. Then the function $z \mapsto\left(1,\left(z-L_{p}\right)^{-1} 1\right)$ has a meromorphic continuation from the half-plane $\operatorname{Re} z>m_{p}$ to the the half-plane $\operatorname{Re} z>e(\alpha)-\gamma$ and its only singularity there is a simple pole at $z=e(\alpha)$ with residue $\mathrm{e}^{c}$. Moreover, for any $\epsilon>0$ and $j \in\{0,1\}$,

$$
\sup _{x>e(\alpha)-\gamma+\epsilon} \int_{|y|>\epsilon}\left|\left(1,\left(x+\mathrm{i} y-L_{p}\right)^{j-2} 1\right)\right|^{j+1} \mathrm{~d} y<\infty .
$$


This result has the following converse:

Proposition 5.17 Suppose that the function $z \mapsto\left(1,\left(z-L_{p}\right)^{-1} 1\right)$ has a meromorphic continuation from the halfplane $\operatorname{Re} z>m_{p}$ to the half-plane $\operatorname{Re} z>e(\alpha)-\gamma$ for some $\gamma>0$ and that its only singularity there is a simple pole at $z=e(\alpha)$. Suppose also that for some $\epsilon>0$ and any $j \in\{0,1\}$,

$$
\sup _{x>e(\alpha)-\gamma} \int_{|y|>\epsilon}\left|\left(1,\left(x+\mathrm{i} y-L_{p}\right)^{j-2} 1\right)\right|^{j+1} \mathrm{~d} y<\infty .
$$

Then

$$
e_{t}(\alpha)=t e(\alpha)+c+O\left(\mathrm{e}^{-\gamma t}\right)
$$

as $t \rightarrow \infty$.

The proofs of Propositions 5.16 and 5.17 are standard (see [JP3]) and for reasons of space we will omit them.

After introduction of a suitable transfer operator the resonance interpretation of $g(X, Y)$ is very similar.

In the discrete time case (recall (3.19) $)$ instead of the resolvent $\left(z-L_{p}\right)^{-1}$ one considers

$$
\mathcal{R}(z)=\sum_{n=0}^{\infty} \mathrm{e}^{-n z}\left(1, U_{p}^{n} 1\right)
$$

Propositions 5.16 and 5.17 hold in the discrete case after obvious modifications.

The reader familiar with classical results in spectral theory of Ruelle transfer operators [Bo2, Ru1, Ba1, BKL, GL1, GL2, Ba2, Ba3] might be surprised at our insistence on the Hilbert space framework. It is however precisely in this framework and through the link with Tomita-Takesaki theory [BR] that Ruelle transfer operators $\left(L^{p_{-}}\right.$ Liouvilleans) naturally extend to the non-commutative setting. The Banach space framework, which is dominant in the classical presentations, emerges through the complex spectral deformation technique which is a natural tool to study resonances of the $L^{p}$-Liouvilleans in the non-commutative setting (see [JP3] for the case $p=\infty$ ).

\subsection{Examples}

\subsubsection{The microcanonical ideal gas}

In this section we investigate the large time limit in the example of Section 3.6.1, For $F \neq 0$ it follows from Equ. (3.24) that

$$
\lim _{t \rightarrow \infty} \sigma_{t}=(N-1) \frac{|F|}{\sqrt{\epsilon}}
$$

holds for $\omega$-a.e. $(L, \theta) \in M$, hence

$$
\langle\sigma\rangle_{+}=(N-1) \frac{|F|}{\sqrt{\epsilon}}>0 .
$$

The generating function (3.25) can be expressed in terms of the associated Legendre function $P_{n}^{m}$ as

$$
e_{t}(\alpha)=\log \left(\Gamma(N / 2)\left(\frac{2}{\operatorname{sh} \mu t}\right)^{(N-2) / 2} P_{(N-1)|\alpha-1 / 2|-1 / 2}^{-(N-2) / 2}(\operatorname{ch} \mu t)\right) .
$$

From the asymptotic behavior for $z \rightarrow+\infty$ (see e.g. Equ. (8.766) in [GR])

$$
P_{n}^{m}(z)=\frac{1}{\sqrt{\pi}}\left(\frac{\Gamma(n+1 / 2)}{\Gamma(1+n-m)}(2 z)^{n}+\frac{\Gamma(-n-1 / 2)}{\Gamma(-n-m)}(2 z)^{-n-1}\right)\left(1+O\left(z^{-2}\right)\right),
$$


we obtain the ES-functional

$$
e(\alpha)=\lim _{t \rightarrow+\infty} \frac{1}{t} e_{t}(\alpha)=-\langle\sigma\rangle_{+}\left(\frac{1}{2}-\left|\alpha-\frac{1}{2}\right|\right) .
$$

This function is not differentiable at $\alpha=1 / 2$. However, it is differentiable near $\alpha=0$, we conclude that the entropy production observable satisfies a (local) Large Deviation Principle with rate function

$$
I(s)= \begin{cases}-\infty & \text { if }|s|>\langle\sigma\rangle_{+}, \\ \frac{1}{2}\left(s-\langle\sigma\rangle_{+}\right) & \text {if }|s| \leq\langle\sigma\rangle_{+},\end{cases}
$$

near its mean value $\langle\sigma\rangle_{+}$.

With $F$ as control parameter, we have the flux relation $\sigma=F(N-1) \epsilon^{-1 / 2} \operatorname{th} \xi$, and hence the flux observable

$$
\Phi=\frac{N-1}{\sqrt{\epsilon}} \operatorname{th} \xi .
$$

From Equ. (5.55) we conclude that $\langle\Phi\rangle_{+}=(N-1) \epsilon^{-1 / 2} \operatorname{sign} F$ is not differentiable at $F=0$ and linear response theory fails for this model. We remark that the finite time Green-Kubo formula reads

$$
L_{t}=\left.\partial_{F}\left(\frac{1}{t} \int_{0}^{t} \omega\left(\Phi_{s}\right) \mathrm{d} s\right)\right|_{F=0}=\frac{1}{2} \int_{-t}^{t} \omega\left(\Phi \Phi_{s}\right)\left(1-\frac{|s|}{t}\right) \mathrm{d} s=\frac{t}{2} \omega\left(\Phi^{2}\right)=\frac{(N-1)^{2}}{N} \frac{t}{2 \epsilon},
$$

and that $L_{t}$ diverges as $t \rightarrow \infty$.

\subsubsection{The harmonic chain}

We continue with the example of Section 4.4.1. We again omit the details of the calculations which the interested reader may find in [JLTP].

Since the reservoirs in Section 4.4.1 are all finite, one has

$$
\left\langle\Phi^{(L / R)}\right\rangle_{+}=\lim _{t \rightarrow \infty} \frac{1}{t} \int_{0}^{t} \omega_{X}\left(\Phi_{s}^{(L / R)}\right) \mathrm{d} s=\lim _{t \rightarrow \infty} \frac{1}{2 t} \operatorname{tr}\left(D_{X}\left(h_{L / R}-\mathrm{e}^{t \mathcal{L}^{*}} h_{L / R} \mathrm{e}^{t \mathcal{L}}\right)\right)=0 .
$$

In particular, $\left\langle\sigma_{X}\right\rangle_{+}=X_{L}\left\langle\Phi^{(L)}\right\rangle_{+}+X_{R}\left\langle\Phi^{(R)}\right\rangle_{+}=0$.

To get a non-vanishing entropy production, we must perform the thermodynamic limit of the reservoirs (i.e., take $n \rightarrow \infty$ keeping $m$ fixed) before taking $t \rightarrow \infty$. We shall not be concerned here with the existence of a limiting dynamical system. However, it should be clear from our discussion that the limiting dynamical system exists as a special instance of the Gaussian dynamical systems of Section 9 . In the following, we denote the dependence on $n$ of various objects of interest by the superscript ${ }^{(n)}$.

The phase space of the composite system has a natural embedding in the real Hilbert space $\mathcal{H}=\ell_{\mathbb{R}}^{2}(\mathbb{Z}) \oplus \ell_{\mathbb{R}}^{2}(\mathbb{Z})$. We denote by $\mathcal{H}=\mathcal{H}_{L} \oplus \mathcal{H}_{S} \oplus \mathcal{H}_{R}$ the decomposition of this space corresponding to the partition

$$
\mathbb{Z}=\{x \in \mathbb{Z} \mid x<-m\} \cup\{x \in \mathbb{Z} \mid-m \leq x \leq m\} \cup\{x \in \mathbb{Z} \mid x>m\},
$$

and by $p_{L}, p_{\mathcal{S}}, p_{R}$ the corresponding orthogonal projections. The operators $h^{(n)}, h_{L}^{(n)}, h_{R}^{(n)}$ and $k^{(n)}(X)$ have strong limits in this Hilbert space as $n \rightarrow \infty$. We shall denote these bounded self-adjoint limits by $h, h_{L}, h_{R}$ and $k(X)$. For example

$$
\mathrm{s}-\lim _{n \rightarrow \infty} h^{(n)}=h=\left(\begin{array}{cc}
I & 0 \\
0 & I-\Delta
\end{array}\right),
$$


where $\Delta$ is the finite difference Laplacian on $\ell^{2}(\mathbb{Z})$

$$
(\Delta u)_{x}=u_{x-1}-2 u_{x}+u_{x+1} .
$$

In the same way, the generators $\mathcal{L}_{0}^{(n)}, \mathcal{L}^{(n)}$ as well as their adjoints $\mathcal{L}_{0}^{(n) *}, \mathcal{L}^{(n) *}$ have bounded strong limits $\mathcal{L}_{0}, \mathcal{L}$ and $\mathcal{L}_{0}^{*}, \mathcal{L}^{*}$. It follows that

$$
\mathrm{s}-\lim _{n \rightarrow \infty} \mathrm{e}^{t \mathcal{L}_{0}^{(n)}}=\mathrm{e}^{t \mathcal{L}_{0}}, \quad \mathrm{~s}-\lim _{n \rightarrow \infty} \mathrm{e}^{t \mathcal{L}^{(n)}}=\mathrm{e}^{t \mathcal{L}},
$$

and similar relations for the adjoint groups hold uniformly on compact time intervals.

Denote by $\phi_{L / R}$ the finite rank self-adjoint operator associated to the quadratic form $2 \Phi^{(L / R)}$ (recall that it does not depend on $n$ ). For $Y=\left(Y_{L}, Y_{R}\right) \in \mathbb{R}^{2}$ set $\phi(Y)=Y_{L} \phi_{L}+Y_{R} \phi_{R}$. It follows from

$$
\mathrm{e}^{t \mathcal{L}^{(n) *}} k^{(n)}(Y) \mathrm{e}^{t \mathcal{L}^{(n)}}-k^{(n)}(Y)=-\int_{0}^{t} \mathrm{e}^{s \mathcal{L}^{(n) *}} \phi(Y) \mathrm{e}^{s \mathcal{L}^{(n)}} \mathrm{d} s,
$$

that

$$
\lim _{n \rightarrow \infty} \mathrm{e}^{t \mathcal{L}^{(n) *}} k^{(n)}(Y) \mathrm{e}^{t \mathcal{L}^{(n)}}-k^{(n)}(Y)=\mathrm{e}^{t \mathcal{L}^{*}} k(Y) \mathrm{e}^{t \mathcal{L}}-k(Y)=-\int_{0}^{t} \mathrm{e}^{s \mathcal{L}^{*}} \phi(Y) \mathrm{e}^{s \mathcal{L}} \mathrm{d} s,
$$

holds in the trace norm for any finite $t \in \mathbb{R}$. Since $\left(\beta h^{(n)}-k^{(n)}(X)\right)^{-1}$ is uniformly bounded and

$$
\mathrm{s}-\lim _{n \rightarrow \infty}\left(\beta h^{(n)}-k^{(n)}(X)\right)^{-1}=(\beta h-k(X))^{-1},
$$

we conclude that

$$
\lim _{n \rightarrow \infty}\left(\beta h^{(n)}-k^{(n)}(X)\right)^{-1}\left(\mathrm{e}^{t \mathcal{L}^{(n) *}} k^{(n)}(Y) \mathrm{e}^{t \mathcal{L}^{(n)}}-k^{(n)}(Y)\right)=-\int_{0}^{t}(\beta h-k(X))^{-1} \mathrm{e}^{s \mathcal{L}^{*}} \phi(Y) \mathrm{e}^{s \mathcal{L}} \mathrm{d} s,
$$

holds in trace norm. This finally yields

$$
g_{t}(X, Y)=\lim _{n \rightarrow \infty} g_{t}^{(n)}(X, Y)=-\frac{1}{2} \log \operatorname{det}\left(I+\int_{0}^{t}(\beta h-k(X))^{-1} \mathrm{e}^{s \mathcal{L}^{*}} \phi(Y) \mathrm{e}^{s \mathcal{L}} \mathrm{d} s\right),
$$

with the convention that $\log x=-\infty$ for $x \leq 0$.

We are now in position to perform the $t \rightarrow \infty$ limit. The wave operators

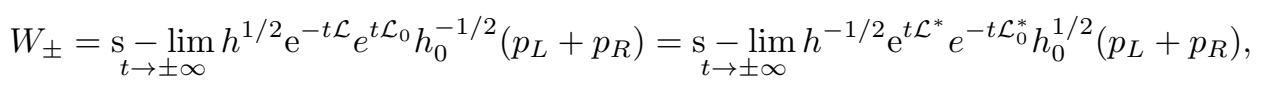

exists and are partial isometries from $\mathcal{H}_{L} \oplus \mathcal{H}_{R}$ to $\mathcal{H}$. The scattering matrix $S=W_{+}^{*} W_{-}$is unitary on $\mathcal{H}_{L} \oplus \mathcal{H}_{R}$ and commutes with the self-adjoint operator $L_{0}=h_{0}^{1 / 2} \mathcal{L}_{0} h_{0}^{-1 / 2}$. Denoting by $\mathcal{H}_{L} \oplus \mathcal{H}_{R}=\int{ }^{\oplus} \mathfrak{h}(\lambda) \mathrm{d} \lambda$ the spectral decomposition induced by $L_{0}$ and by $S(\lambda)$ the fiber of the scattering matrix $S$ acting on $\mathfrak{h}(\lambda)$, one has

$$
g(X, Y)=\lim _{t \rightarrow \infty} \frac{1}{t} g_{t}(X, Y)=-\frac{1}{4 \pi} \int \log \operatorname{det}_{\mathfrak{h}(\lambda)}\left(I-(\beta I-\widehat{X})^{-1}\left(S(\lambda)^{*} \widehat{Y} S(\lambda)-\widehat{Y}\right)\right) \mathrm{d} \lambda,
$$

where $\widehat{X}=X_{L} p_{L}+X_{R} p_{R}$. We note that this formula remains valid for arbitrary finite harmonic system coupled to a finite number of infinite harmonic reservoirs, as long as the coupling $v=h-h_{0}$ is trace class (see [JLTP]). The fluxes are given by

$$
\left\langle\Phi^{(j)}\right\rangle_{+}=-\left.\partial_{Y_{j}} g(X, Y)\right|_{Y=0}=\frac{1}{4 \pi} \int \operatorname{tr}_{\mathfrak{h}(\lambda)}\left((\beta I-\widehat{X})^{-1}\left(p_{j}-S(\lambda)^{*} p_{j} S(\lambda)\right)\right) \mathrm{d} \lambda,
$$


which is a classical version of the Büttiker-Landauer formula (see [AJPP]).

Explicit calculation of the scattering matrix yields the result

$$
g(X, Y)=-\kappa \log \left(\frac{\left(\left(\beta-X_{L}\right)-\left(Y_{R}-Y_{L}\right)\right)\left(\left(\beta-X_{R}\right)+\left(Y_{R}-Y_{L}\right)\right)}{\left(\beta-X_{L}\right)\left(\beta-X_{R}\right)}\right), \quad \kappa=\frac{\sqrt{5}-1}{2 \pi} .
$$

For fixed $X \in \mathbb{R}^{2}$ such that $\max \left(X_{L}, X_{R}\right)<\beta$, the function $Y \mapsto g(X, Y)$ is a real analytic in the open strip $\left\{Y \in \mathbb{R}^{2} \mid-\left(\beta-X_{R}\right)<Y_{R}-Y_{L}<\beta-X_{L}\right\}$.

By Proposition 5.11

$$
\left\langle\Phi^{(L)}\right\rangle_{+}=-\left\langle\Phi^{(R)}\right\rangle_{+}=\kappa\left(\frac{1}{\beta-X_{L}}-\frac{1}{\beta-X_{R}}\right)=\kappa\left(T_{L}-T_{R}\right),
$$

where $T_{L / R}=\left(\beta-X_{L / R}\right)^{-1}$ denotes the temperature of the $L / R$ reservoir. In particular, entropy production

$$
\left\langle\sigma_{X}\right\rangle_{+}=X_{L}\left\langle\Phi^{(L)}\right\rangle_{+}+X_{R}\left\langle\Phi^{(R)}\right\rangle_{+}=\kappa \frac{\left(X_{L}-X_{R}\right)^{2}}{\left(\beta-X_{L}\right)\left(\beta-X_{R}\right)}=\kappa \frac{\left(T_{L}-T_{R}\right)^{2}}{T_{L} T_{R}}
$$

is strictly positive provided $T_{L} \neq T_{R}$. By Proposition 5.11, the flux observables $\left(\Phi^{(L)}, \Phi^{(R)}\right)$ satisfy a large deviation principle w.r.t. $\omega_{X}$, with rate function

$$
I_{X}\left(s_{L}, s_{R}\right)= \begin{cases}-\infty & \text { if } s_{L}+s_{R} \neq 0 \\ F(\theta) & \text { if } s_{L}=-s_{R}=\frac{\kappa}{\beta_{0}} \operatorname{sh} \theta\end{cases}
$$

where

$$
F(\theta)=-\kappa\left[2 \operatorname{sh}^{2} \frac{\theta}{2}-\frac{\delta}{\beta_{0}} \operatorname{sh} \theta-\log \left(\left(1-\frac{\delta^{2}}{\beta_{0}^{2}}\right) \operatorname{ch}^{2} \frac{\theta}{2}\right)\right],
$$

$\beta_{0}=\beta-\left(X_{L}+X_{R}\right) / 2$ and $\delta=\left(X_{L}-X_{R}\right) / 2$.

Writing Equ. (5.56) as $g_{t}(X, Y)=-\frac{1}{2} \log \operatorname{det}\left(I+A_{t}\right)$, one easily shows that the trace norm of $A_{t}$ is bounded by

$$
\left\|A_{t}\right\|_{1} \leq C|Y||t|
$$

while its operator norm satisfies

$$
\left\|A_{t}\right\| \leq C|Y|
$$

for any $Y \in \mathbb{C}^{2}$ and $t \in \mathbb{R}$ with a constant $C$ depending only on $\beta$ and $X$. It follows that the bound 5.51) is satisfied for sufficiently small $\epsilon>0$. Finally, as a consequence of the local decay estimate for the discrete Klein-Gordon equation

$$
\left|\left(\delta_{x}, \mathrm{e}^{-\mathrm{i} t \sqrt{-\Delta+1}} \delta_{y}\right)\right| \leq C_{x, y}|t|^{-1 / 2}
$$

Hypothesis (T5) is satisfied. It follows from assertions (3) and (4) of Proposition 5.15 that the fluctuation dissipation theorem holds.

The equality $\left\langle\Phi^{(L)}\right\rangle_{+}=-\left\langle\Phi^{(R)}\right\rangle_{+}$in $(5.58)$ is a consequence of energy conservation. More generally, for an open system as described in Section 4.4, energy conservation implies $\sum_{j}\left\langle\Phi^{(j)}\right\rangle_{+}=0$. For the same reason, the rate function $I_{X}(s)$ takes the value $-\infty$ outside of the subspace $\sum_{j} s_{j}=0$ and the covariance matrix $\mathbf{D}$ in the central limit theorem is singular on this subspace. One can easily avoid all these singularities by reducing the number of parameters. In fact, one observes that the large time characteristics of the system do not depend on the initial inverse temperature $\beta$ of the small subsystem $S$. Indeed, the GES-functional $g(X, Y)$ only depends on the inverse temperatures of the reservoirs $\beta-X_{j}$ (this is a general feature of open systems). This suggests to fix the parameter 
$\beta$ at the mean inverse temperature of the reservoirs by restricting the parameters $X$ to the hyperplane $\sum_{j} X_{j}=0$ of $\mathbb{R}^{N}$. This reduces the number of parameters and consequently the number of associated fluxes by one. In our simple example with two reservoirs, this amounts to set $X_{L}=-X_{R}$.

Harmonic systems are very special in that the study of their dynamics can be effectively reduced to an application of the trace class scattering theory. The more difficult case of a finite anharmonic chain coupled to infinitely extended harmonic reservoirs has been analyzed in [EPR1, EPR2, EH1, EH2, RT1, RT2].

\section{Non-equilibrium steady states}

\subsection{Basic notions}

To discuss Non-Equilibrium Steady States (NESS) we need several additional assumptions on $(M, \phi, \omega)$. The first is:

(NESS1) $M$ is a complete separable metric space.

In this case it is natural to equip $\mathcal{S}$ with the topology of weak convergence, i.e., the minimal topology w.r.t. which all the functionals

$$
\mathcal{S} \ni \nu \mapsto \nu(f), \quad f \in C(M),
$$

are continuous. This topology is metrizable and $\mathcal{S}$ is a complete separable metric space. A sequence $\nu_{n} \in \mathcal{S}$ converges to $\nu$ iff $\nu_{n}(f) \rightarrow \nu(f)$ for all $f \in C(M)$.

With regard to Theorem 2.1 in (1) $B_{\mathbb{R}}(M)$ could be replaced by $C_{\mathbb{R}}(M)$. (6) and (7) are valid as formulated except that in (6) the convergent nets can be replaced with convergent sequences.

$\mathcal{S}$ is compact iff $M$ is compact. More generally, a set $\mathfrak{S} \subset \mathcal{S}$ is precompact (its closure is compact) iff $\mathfrak{S}$ is tight, i.e., for any $\epsilon>0$ there is a compact set $K_{\epsilon} \subset M$ such that $\nu\left(K_{\epsilon}\right)>1-\epsilon$ for all $\nu \in \mathfrak{S}$.

The remaining additional assumptions are:

(NESS2) $\phi^{t}$ is a group of homeomorphisms of $M$ and the map $(t, x) \mapsto \phi^{t}(x)$ is continuous.

(NESS3) $\sigma \in C(M)$.

(NESS4) The set of states

$$
\mathfrak{S}(\omega)=\left\{\frac{1}{t} \int_{0}^{t} \omega_{s} \mathrm{~d} s \mid t \geq 1\right\}
$$

is precompact in $\mathcal{S}$.

We denote by $\mathcal{S}_{+}(\omega)$ the set of limit points of $\mathfrak{S}(\omega)$ as $t \rightarrow \infty$. $\mathcal{S}_{+}(\omega)$ is non-empty and $\omega_{+} \in \mathcal{S}_{+}(\omega)$ iff there exists a sequence $t_{n} \rightarrow \infty$ such that, for all $f \in C(M)$,

$$
\lim _{n \rightarrow \infty} \frac{1}{t_{n}} \int_{0}^{t_{n}} \omega_{s}(f) \mathrm{d} s=\omega_{+}(f) .
$$

Definition 6.1 We shall call the elements of $\mathcal{S}_{+}(\omega)$ the NESS of $(M, \phi, \omega)$.

Two basic properties of a NESS $\omega_{+}$are: 
Proposition 6.2 (1) $\omega_{+} \in \mathcal{S}_{I}$.

(2) $\omega_{+}(\sigma) \geq 0$.

Proof. The statements follow from the relations (6.59) and (3.7).

The assumptions (NESS1)-(NESS4) naturally apply to a family $\left(M, \phi_{X}, \omega_{X}, \boldsymbol{\Phi}_{X}\right)$ except that in this case in (NESS3) one also requires that $\Phi_{X}^{(j)} \in C(M)$.

Remark 1. In the study of specific models it is an important and often very difficult problem to prove that $\mathcal{S}_{+}(\omega)$ is a singleton, namely that there exists $\omega_{+} \in \mathcal{S}$ such that for all $f \in C(M)$,

$$
\lim _{t \rightarrow \infty} \frac{1}{t} \int_{0}^{t} \omega_{s}(f) \mathrm{d} s=\omega_{+}(f) .
$$

Remark 2. The regularity assumptions (NESS2) and (NESS3) are made for simplicity of presentations and can be relaxed, see Sections 6.4.2 and 9 for examples.

Remark 3. The NESS property is related to the SRB property in dynamical systems, see [Ru2] for more details.

\subsection{The Gallavotti-Cohen Fluctuation Theorem}

Let $(M, \phi, \omega)$ be a TRI system and let $\omega_{+} \in \mathcal{S}_{+}(\omega)$ be given. Let

$$
e_{t+}(\alpha)=\log \omega_{+}\left(\mathrm{e}^{-\alpha \int_{0}^{t} \sigma_{s} \mathrm{~d} s}\right) .
$$

Note that $e_{t+}(\alpha)$ is a convex function of the parameter $\alpha$ and that $e_{t+}(\alpha) \geq-\alpha t \omega_{+}(\sigma)$.

We suppose:

(GC) The Gallavotti-Cohen functional (GC-functional)

$$
e_{+}(\alpha)=\lim _{t \rightarrow \infty} \frac{1}{t} e_{t+}(\alpha),
$$

exists for all $\alpha \in \mathbb{R}$.

If the GC-functional satisfies

$$
e_{+}(\alpha)=e_{+}(1-\alpha)
$$

for all $\alpha$, we shall say that the GC-symmetry holds. $e_{+}(\alpha)$ is a convex function and the GC-symmetry implies that $e_{+}(0)=e_{+}(1)=0, e_{+}(\alpha) \leq 0$ for $\alpha \in[0,1]$, and $e_{+}(\alpha) \geq 0$ for $\alpha \notin[0,1]$.

In comparison with the ES-symmetry, we remark that in general the relation $e_{t+}(\alpha)=e_{t+}(1-\alpha)$ does not hold for finite $t$ and that GC-symmetry may fail even in some very simple models (see Subsection 8.3). In contrast, whenever $e(\alpha)$ exists, the universally valid finite time ES-symmetry $e_{t}(\alpha)=e_{t}(1-\alpha)$ implies that $e(\alpha)=$ $e(1-\alpha)$.

Proposition 6.3 (1) Suppose that $e_{+}(\alpha)$ is differentiable at $\alpha=0$. Then (E3) holds and

$$
\langle\sigma\rangle_{+}=\omega_{+}(\sigma)=\lim _{t \rightarrow \infty} \frac{1}{t} \int_{0}^{t} \omega\left(\sigma_{s}\right) \mathrm{d} s=-e_{+}^{\prime}(0) .
$$


If in addition the GC-symmetry holds, then $\langle\sigma\rangle_{+}=0$ iff $e_{+}(\alpha)=0$ for $\alpha \in[0,1]$. For any regular sequence $t_{n}$

$$
\lim _{n \rightarrow \infty} \frac{1}{t_{n}} \int_{0}^{t_{n}} \sigma_{s}(x) \mathrm{d} s=\langle\sigma\rangle_{+},
$$

for $\omega_{+}$-a.e. $x \in M$. If $\langle\sigma\rangle_{+}>0$, then $\omega$ and $\omega_{+}$are mutually singular.

(2) Suppose that $e_{+}(\alpha)$ is differentiable for all $\alpha$. Then the Large Deviation Principle holds for $\sigma$ w.r.t. $\left(M, \phi, \omega_{+}\right)$ with the concave rate function $I_{+}(s)=\inf _{\alpha \in \mathbb{R}}\left(\alpha s+e_{+}(\alpha)\right)$. If the GC-symmetry holds, then

$$
I_{+}(s)=s+I_{+}(-s) .
$$

The last relation is called the GC-symmetry for the rate function $I_{+}$.

Proof. The only part that requires a proof is the last statement in Part (1). Suppose that $\left.\langle\sigma\rangle_{+}\right\rangle 0$ and let $\omega_{+}=\nu_{1}+\nu_{2}, \nu_{1} \ll \omega, \nu_{2} \perp \omega$, be the Radon-Nikodym decomposition of $\omega_{+}$w.r.t. $\omega$. Since $\omega_{+} \in \mathcal{S}_{I}$, Assumption (C) and the uniqueness of the Radon-Nikodym decomposition imply that $\nu_{1}, \nu_{2} \in \mathcal{S}_{I}$. If $\nu_{1}$ is nontrivial, then Corollary 5.5 implies that $\langle\sigma\rangle_{+}=0$, a contradiction.

Consider a family $\left(M, \phi_{X}, \omega_{X}, \boldsymbol{\Phi}_{X}\right), X \in \mathbb{R}^{N}$, satisfying (T1) (T2) and let $\omega_{X+} \in \mathcal{S}_{+}\left(\omega_{X}\right)$ be given. Let

$$
g_{t+}(X, Y)=\log \omega_{X+}\left(\mathrm{e}^{-Y \cdot \int_{0}^{t} \boldsymbol{\Phi}_{X s} \mathrm{~d} s}\right) .
$$

We suppose:

(GGC) The Generalized Gallavotti-Cohen functional (GGC-functional)

$$
g_{+}(X, Y)=\lim _{t \rightarrow \infty} \frac{1}{t} g_{t+}(X, Y)
$$

exists for all $X, Y$.

If the GGC-functional satisfies

$$
g_{+}(X, Y)=g_{+}(X, X-Y),
$$

for all $X, Y$, we shall say that $G G C$-symmetry holds.

Proposition 6.4 (1) Suppose that $Y \mapsto g_{+}(X, Y)$ is differentiable at $Y=0$. Then

$$
\left\langle\boldsymbol{\Phi}_{X}\right\rangle_{+}=\lim _{t \rightarrow \infty} \frac{1}{t} \int_{0}^{t} \omega_{X}\left(\boldsymbol{\Phi}_{X s}\right) \mathrm{d} s
$$

exists and $\left\langle\boldsymbol{\Phi}_{X}\right\rangle_{+}=\omega_{X+}\left(\boldsymbol{\Phi}_{X}\right)=-\left.\nabla_{Y} g_{+}(X, Y)\right|_{Y=0}$. Moreover, for any regular sequence $t_{n}$,

$$
\lim _{n \rightarrow \infty} \frac{1}{t_{n}} \int_{0}^{t_{n}} \boldsymbol{\Phi}_{X s}(x) \mathrm{d} s=\omega_{X+}\left(\boldsymbol{\Phi}_{X}\right),
$$

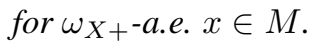

(2) Suppose that $Y \mapsto g_{+}(X, Y)$ is differentiable for all $Y$. Then the Large Deviation Principle holds for $\boldsymbol{\Phi}_{X}$ w.r.t. $\left(M, \phi_{X}, \omega_{X+}\right)$ with the concave rate function $I_{X+}(s)=\inf _{Y \in \mathbb{R}^{N}}\left(Y \cdot s+g_{+}(X, Y)\right)$. If the GGC-symmetry holds, then

$$
I_{X+}(s)=X \cdot s+I_{X+}(-s) .
$$


(3) Suppose that $g_{+}(X, Y)$ is $C^{1,2}$ in a neighborhood of $(0,0)$ and that the $G G C$-symmetry holds. Then the kinetic transport coefficients are defined and satisfy the Onsager reciprocity relations $L_{j k}=L_{k j}$.

(4) In addition to the assumptions of (3) suppose that for some $\epsilon>0$,

$$
\sup _{Y \in D_{\epsilon}, t>1} \frac{1}{t}\left|g_{t+}(0, Y)\right|<\infty .
$$

Then the Fluctuation-Dissipation Theorem holds.

The proof of this proposition is the same as the proof of Proposition 6.4. Apart from the last statement in (1) and (2), the conclusions of these two propositions are also identical. Note that $g_{t+}(0, Y)=g_{t}(0, Y)$ and so the parts (4) of the two propositions are in fact identical (we included the statement for completeness).

We shall say that a given TRI system satisfies the Gallavotti-Cohen Fluctuation Theorem if the respective functionals $e_{+}(\alpha) / g_{+}(X, Y)$ exist and are differentiable/ $C^{1,2}$ and satisfy the GC/GGC-symmetry. It follows from Propositions 6.3 and 6.4 that the Gallavotti-Cohen Fluctuation Theorem is also an extension of the fluctuationdissipation theorem to the far from equilibrium region.

\subsection{The resonance interpretation of $e_{+}(\alpha)$}

Let $\omega_{+}$be a NESS of $(M, \phi, \omega)$ (in particular, we assume that (NESS1)-(NESS4) hold). For $p \in(-\infty, \infty], p \neq 0$ and $f \in C(M)$ let

$$
U_{p}(t) f=\mathrm{e}^{\frac{1}{p} \int_{0}^{t} \sigma_{-s}} f_{-t} .
$$

One easily shows that:

Proposition 6.5 (1) $\omega_{+}\left(\left[U_{p}(t) f\right]\left[U_{-p}(t) g\right]\right)=\omega_{+}(f g)$.

(2) $U_{p}(t)$ extends to a strongly continuous group of bounded operators on $L^{2}\left(M, \mathrm{~d} \omega_{+}\right)$such that $U_{p}^{*}(t)=$ $U_{-p}(-t)$ and

$$
\left\|U_{p}(t)\right\| \leq \mathrm{e}^{|t| m_{p+}}
$$

where $m_{p+}=\sup _{x \in M}|\sigma(x)| /|p|$. Let $L_{p+}$ be the generator of $U_{p}(t), U_{p}(t)=\mathrm{e}^{t L_{p+}}$. Then $L_{p+}^{*}=-L_{-p+}$, $\operatorname{sp}\left(L_{p+}\right) \subset\left\{z|| \operatorname{Re} z \mid \leq m_{p+}\right\}$, $\operatorname{Dom}\left(L_{p+}\right)=\operatorname{Dom}\left(L_{\infty+}\right)$, and for $f \in \operatorname{Dom} L_{p+}$

$$
L_{p+} f=L_{\infty+} f+\frac{\sigma}{p} f .
$$

We shall call the operator $L_{p+}$ the NESS $L^{p}$-Liouvillean. If $\alpha=-1 / p$, then

$$
e_{t+}(\alpha)=\log \left(1, \mathrm{e}^{t L_{p+}} 1\right)_{+}=\log \int_{M} \mathrm{e}^{t L_{p+}} 1 \mathrm{~d} \omega_{+} .
$$

Under suitable regularity condition this relation leads to the identification of $e_{+}(\alpha)$ with a complex resonance of $L_{p+}$. With the obvious notational changes Propositions 5.16 and 5.17 apply to $e_{+}(\alpha)$ and $L_{p+}$. 


\subsection{Examples}

\subsubsection{The microcanonical ideal gas}

In the example of Section 3.6.1, it follows from (3.22), (3.23) that the unique NESS of the system is

$$
\mathrm{d} \omega_{+}=\prod_{j=1}^{N} \delta\left(L_{j}-\sqrt{\epsilon}\right) \frac{\mathrm{d} L_{j} \mathrm{~d} \theta_{j}}{2 \pi} .
$$

We note that it is singular w.r.t. the reference measure $\omega$. One immediately computes

$$
e_{+t}(\alpha)=-\alpha t\langle\sigma\rangle_{+}
$$

and observes that the GC-functional

$$
e_{+}(\alpha)=-\alpha\langle\sigma\rangle_{+},
$$

does not satisfy the GC-symmetry. Entropy production does not fluctuate in the NESS $\omega_{+}$. Accordingly, the rate function for its large deviation is

$$
I_{+}(s)= \begin{cases}0 & \text { if } s=\langle\sigma\rangle_{+} \\ -\infty & \text { otherwise }\end{cases}
$$

The GC-fluctuation theorem fails in this model.

\subsubsection{The harmonic chain}

To compute the NESS in the example of Section 5.5.2 (in the thermodynamic limit) we note that $\omega_{X t}$ is the centered Gaussian measure of covariance

$$
D_{t}=\left(\mathrm{e}^{-t \mathcal{L}^{*}}(\beta h-k(X)) \mathrm{e}^{-t \mathcal{L}}\right)^{-1}=h^{-1 / 2}\left(\beta-h^{-1 / 2} \mathrm{e}^{-t \mathcal{L}^{*}} h_{0}^{1 / 2} \widehat{X} h_{0}^{1 / 2} \mathrm{e}^{-t \mathcal{L}} h^{-1 / 2}\right)^{-1} h^{-1 / 2} .
$$

By Equ. (5.57), this covariance converges strongly to the limit

$$
D_{+}=h^{-1 / 2} W_{-}(\beta-\widehat{X})^{-1} W_{-}^{*} h^{-1 / 2} .
$$

It follows that the system has a unique NESS $\omega_{X+}$ which is Gaussian with covariance $D_{+}$. In particular, one has

$$
g_{t+}(X, Y)=-\frac{1}{2} \log \operatorname{det}\left(I+\int_{0}^{t} D_{+} \mathrm{e}^{s \mathcal{L}^{*}} \phi(Y) \mathrm{e}^{s \mathcal{L}} \mathrm{d} s\right) .
$$

As for the functional $g_{t}(X, Y)$, one can compute the infinite time limit and get (see [JLTP])

$$
g_{+}(X, Y)=\lim _{t \rightarrow \infty} \frac{1}{t} g_{t+}(X, Y)=g(X, Y),
$$

i.e., the GGC-functional coincide with the GES-functional. In particular, it satisfies the GGC-symmetry and the Gallavotti-Cohen fluctuation theorem holds. Note that in this example Assumptions (NESS2) and (NESS3) do not hold. 


\section{The principle of regular entropic fluctuations}

The mathematical similarity between Propositions 5.14, 5.15 on one side and Propositions 6.3, 6.4 on the other side is not accidental. The principal distinction is that the Evans-Searles symmetries are universal-they hold for any TRI dynamical systems for which the objects in question are defined. The mechanism behind GallavottiCohen symmetries a priori could be model dependent and in general they may fail. Perhaps surprisingly, a careful look at all principal classes of models for which the symmetries have been rigorously established reveals that the respective functionals satisfy

$$
e(\alpha)=e_{+}(\alpha), \quad g(X, Y)=g_{+}(X, Y) .
$$

This is true for the toy models (see Section 8), for Hamiltonian open systems systems treated in [RT1] (see Section 4.4) and for Anosov diffeomorphisms of compact manifolds [GC1, GC2] (see Section 11). In each single case the strong ergodic properties of the model force the relations (7.61). Note that the first relation in (7.61) holds iff the limits in the expression

$$
e_{+}(\alpha)=\lim _{t \rightarrow \infty} \lim _{u \rightarrow \infty} \frac{1}{t} \log \omega_{u}\left(\mathrm{e}^{-\alpha \int_{0}^{t} \sigma_{s} \mathrm{~d} s}\right)
$$

can be interchanged and a similar remark applies to the second relation. This leads to a transparent mechanism for validity of GC-symmetries: The strong ergodicity (chaoticity) of the model forces the identities (7.61) and the universal ES-symmetries imply that the GC-symmetries hold. This leads to the principle of regular entropic fluctuations.

Definition 7.1 We shall say that $\left(M, \phi, \omega, \omega_{+}\right)$satisfies the principle of regular entropic fluctuations if $e(\alpha)$ and $e_{+}(\alpha)$ exist, are differentiable in a neighborhood of $\alpha=0$, and satisfy

$$
e(\alpha)=e_{+}(\alpha)
$$

for all $\alpha$. In the presence of control parameters, we say that family $\left(M, \phi_{X}, \omega_{X}, \omega_{X+}, \boldsymbol{\Phi}_{X}\right)$ satisfy the principle of regular fluctuations if $g(X, Y)$ and $g_{+}(X, Y)$ exist, are $C^{1,2}$ in a neighborhood of $(0,0)$ and satisfy

$$
g(X, Y)=g_{+}(X, Y) .
$$

Combining Propositions 5.14, 5.15, 6.3, 6.4 one derives the implications of the proposed principle. The principle will naturally extend to quantum statistical mechanics.

We emphasize that the principle of regular entropic fluctuations is ergodic in nature. It would be very interesting to exhibit examples of non-trivial physically relevant models for which the principle fails in the sense that $e(\alpha)$ and $e_{+}(\alpha)$ exist and are differentiable, the GC-symmetry holds, but $e(\alpha) \neq e_{+}(\alpha)$.

If the principle of regular entropic fluctuations holds in a given model, then one may consider the Evans-Searles and Gallavotti-Cohen symmetries as mathematically equivalent. This does not mean that implications of Evans-Searles and Gallavotti-Cohen Fluctuation Theorems are identical. If $(M, \phi, \omega)$ has non vanishing entropy production, then $\omega$ and $\omega_{+}$are mutually singular, and the corresponding Large Deviation Principles (LDPs) are very different statements. The principle only asserts that these LDPs hold with the same rate function and explains the origin of the Gallavotti-Cohen symmetry.

Note that the Evans-Searles fluctuation theorem requires less regularity than the Gallavotti-Cohen fluctuation theorem and in particular does not require the existence of a NESS. This does not make the notion of NESS redundantthe fine studies of systems far from equilibrium are critically centered around the NESS. The situation is somewhat analogous to studies of phase transitions in spin systems-the pressure functional provides some information about the phase transitions but a much finer information is encoded in the set of equilibrium states at the critical temperature. 
In Sections 10 and 11 we shall examine the ergodic mechanism behind the principle of regular entropic fluctuations in the case of chaotic homeomorphisms of compact metric spaces and in particular Anosov diffeomorphisms. Starting with the works [GC1, GC2] these models have been a basic paradigm for any theory of entropic fluctuations.

\section{Toy models}

In this section we discuss a few additional examples for which the Evans-Searles and Gallavotti-Cohen functionals can be computed explicitly.

\subsection{Bernoulli shift}

Let $M=\{0,1\}^{\mathbb{Z}}$. The elements of $M$ are sequences $x=\left(x_{j}\right)_{j \in \mathbb{Z}}, x_{j} \in\{0,1\}$. Let $\nu_{p}$ be the usual Bernoulli measure on $\{0,1\}, \nu_{p}(\{1\})=p, \nu_{p}(\{0\})=1-p$. Let

$$
\omega=\left(\bigotimes_{n=-\infty}^{0} \nu_{p}\right) \otimes\left(\bigotimes_{n=1}^{\infty} \nu_{q}\right)
$$

where $p, q \in] 0,1[$ are given. The dynamics $\phi$ is the left shift

$$
\phi(x)_{j}=x_{j+1} .
$$

The Radon-Nikodym derivative of $\omega_{1}=\omega \circ \phi^{-1}$ w.r.t. $\omega$ is

$$
\Delta_{\omega_{1} \mid \omega}(x)=\frac{\nu_{p}\left(\left\{x_{0}\right\}\right)}{\nu_{q}\left(\left\{x_{0}\right\}\right)}
$$

and the entropy production observable is

$$
\sigma(x)=\log \Delta_{\omega_{1} \mid \omega}(x)=\log \frac{\mathrm{d} \nu_{q}}{\mathrm{~d} \nu_{p}}\left(x_{0}\right)= \begin{cases}\log \frac{q}{p} & \text { if } x_{0}=1, \\ \log \frac{1-q}{1-p} & \text { if } x_{0}=0 .\end{cases}
$$

Note that for all $n \geq 1$,

$$
\omega\left(\sigma_{n}\right)=q \log \frac{q}{p}+(1-q) \log \frac{1-q}{1-p}=-\operatorname{Ent}\left(\nu_{q} \mid \nu_{p}\right) \geq 0,
$$

so that the system is entropy producing,

$$
\langle\sigma\rangle_{+}=-\operatorname{Ent}\left(\nu_{q} \mid \nu_{p}\right)>0
$$

iff $p \neq q$. Similarly, for all $n \geq 1$,

$$
\frac{1}{n} \log \omega\left(\mathrm{e}^{\alpha \sum_{j=1}^{n} \sigma_{-j}}\right)=\log \left[p^{1-\alpha} q^{\alpha}+(1-p)^{1-\alpha}(1-q)^{\alpha}\right]=\operatorname{Ent}_{\alpha}\left(\nu_{q} \mid \nu_{p}\right),
$$

and so the ES-functional exists and is given by

$$
e(\alpha)=\log \left[p^{1-\alpha} q^{\alpha}+(1-p)^{1-\alpha}(1-q)^{\alpha}\right] .
$$


Assuming $p \neq q$, the ES-symmetry $e(\alpha)=e(1-\alpha)$ holds iff $q=1-p$. In particular, if $q \neq 1-p$, the model is not time reversal invariant. If $q=1-p$, then the time reversal is $\vartheta(x)_{j}=1-x_{-j}$.

Let

$$
\omega_{+}=\bigotimes_{n \in \mathbb{Z}} \nu_{q}
$$

$M$ is a compact metric space and for any $f \in C(M)$,

$$
\lim _{n \rightarrow \infty} \omega_{n}(f)=\omega_{+}(f) .
$$

Hence, $\omega_{+}$is the NESS of $(M, \phi, \omega)$. Moreover, for any $n \geq 1$,

$$
\frac{1}{n} \log \omega_{+}\left(\mathrm{e}^{-\alpha \sum_{j=0}^{n-1} \sigma_{j}}\right)=\log \left[q^{1-\alpha} p^{\alpha}+(1-q)^{1-\alpha}(1-p)^{\alpha}\right],
$$

and so the GC-functional is

$$
e_{+}(\alpha)=e(1-\alpha) \text {. }
$$

The GC-symmetry holds iff the ES-symmetry does, and in this case the GC-functional coincide with the ESfunctional.

If $p=1-q$, then one can consider $X=p-1 / 2$ as a control parameter. The respective Fluctuation-Dissipation Theorem follows easily $[\mathrm{Sh}]$.

\subsection{Baker transformation}

Let $M=[0,1[\times[0,1[, \mathrm{~d} \omega=\mathrm{d} x \mathrm{~d} y$ and

$$
\phi(x, y)= \begin{cases}(x / p, y q) & \text { if } x \in[0, p[ \\ ((x-p) / q, p y+q) & \text { if } x \in[p, 1[\end{cases}
$$

where $p, q \in] 0,1\left[\right.$. This model has been studied in [DGT], see also [Do, TG, $\mid$ Sh]. Define a map $T:\{0,1\}^{\mathbb{Z}} \rightarrow M$ by $T\left\{a_{j}\right\}_{j \in \mathbb{Z}}=(x, y)$ where

$$
x=p\left(a_{0}+\sum_{n=1}^{\infty} a_{n} p^{n-\sum_{i=0}^{n-1} a_{i}} q^{\sum_{i=0}^{n-1} a_{i}}\right), \quad y=q\left(a_{-1}+\sum_{n=1}^{\infty} a_{-n-1} q^{n-\sum_{i=0}^{n-1} a_{-i-1}} q^{\sum_{i=0}^{n-1} a_{-i-1}}\right) .
$$

The map $T$ is a mod 0 isomorphism between the Bernoulli shift considered in the previous section and the Baker transformation. Hence, the ES-functional $e(\alpha)$ of these two models are the same. The non-equilibrium steady states of the two models are also related by the map $T$ and their GC-functionals are equal [Sh].

\subsection{Dilation on a half-line}

Let $M=[0, \infty]$ (the compactified positive half-line), $\phi_{\gamma}^{t}(x)=\mathrm{e}^{\gamma t} x$ where $\gamma \in \mathbb{R}$, and

$$
\mathrm{d} \omega=\frac{2}{\pi} \frac{\mathrm{d} x}{1+x^{2}} .
$$

The map $\vartheta(x)=x^{-1}$ is a time-reversal of $\left(M, \phi_{\gamma}, \omega\right)$. The reference state $\omega$ is invariant under $\phi_{0}$ and for $\gamma \neq 0$ the system has a unique NESS

$$
\omega_{\gamma+}= \begin{cases}\delta_{0} & \text { if } \gamma<0 \\ \delta_{\infty} & \text { if } \gamma>0\end{cases}
$$


One has

$$
\Delta_{\omega_{t} \mid \omega}(x)=\mathrm{e}^{-\gamma t} \frac{1+x^{2}}{1+\mathrm{e}^{-2 \gamma t} x^{2}}, \quad \sigma_{\gamma}(x)=-\gamma \frac{1-x^{2}}{1+x^{2}}
$$

from which it follows that $\left\langle\sigma_{\gamma}\right\rangle_{+}=\omega_{\gamma+}\left(\sigma_{\gamma}\right)=|\gamma|$. One easily computes the ES-functional

$$
e(\alpha)= \begin{cases}-\alpha|\gamma| & \text { if } \alpha \leq 1 / 2 \\ -(1-\alpha)|\gamma| & \text { if } \alpha \geq 1 / 2\end{cases}
$$

while the GC-functional is $e_{+}(\alpha)=-\alpha|\gamma|$. Hence, $e(\alpha) \neq e_{+}(\alpha)$ for $\alpha>1 / 2$, the ES-symmetry holds, but GC does not.

Consider $\gamma$ as a control parameter. The associated flux observable $\Phi=\left(x^{2}-1\right) /\left(x^{2}+1\right)$ does not depend on $\gamma$, $\omega(\Phi)=0$ and $\omega_{\gamma+}(\Phi)=\operatorname{sign} \gamma$. The kinetic transport coefficient

$$
L=\left.\partial_{\gamma} \omega_{\gamma+}(\Phi)\right|_{\gamma=0},
$$

is not well defined. On the other hand, $\omega\left(\Phi_{t}\right)=\operatorname{th}(\gamma t / 2)$ is a real analytic function of $\gamma$, the finite time kinetic transport coefficient is well defined, the finite time Green-Kubo formula holds, and

$$
L_{t}=\left.\partial_{\gamma}\left(\frac{1}{t} \int_{0}^{t} \omega\left(\Phi_{s}\right) \mathrm{d} s\right)\right|_{\gamma=0}=\frac{1}{2} \int_{-t}^{t} \omega\left(\Phi \Phi_{s}\right)\left(1-\frac{|s|}{t}\right) \mathrm{d} s=\frac{t}{2} .
$$

Note that, as in the example of Section 5.5.1, $L_{t}$ diverges as $t \rightarrow \infty$. The reader may have noticed the similarity between this toy example and the microcanonical ideal gas of Section 3.6.1 (see also [CG] for a related example).

By slightly modifying this example we can illustrate another point. Consider $\left(M, \phi_{-\gamma^{2}}, \omega\right)$ and let again $\gamma$ be the control parameter. Then

$$
\sigma_{\gamma}(x)=-\gamma^{2} \frac{1-x^{2}}{1+x^{2}}, \quad \Phi_{\gamma}(x)=-\gamma \frac{1-x^{2}}{1+x^{2}}
$$

$\omega_{\gamma+}=\delta_{0}$ for all $\gamma \neq 0$, and the functions $\left\langle\sigma_{\gamma}\right\rangle_{+}=\gamma^{2},\left\langle\Phi_{\gamma}\right\rangle_{+}=\gamma$ are entire analytic. The kinetic transport coefficient is equal to 1 and, since $\Phi_{0} \equiv 0$, the Green-Kubo formula fails. In this example the finite time linear response theory holds with $L_{t}=0, \gamma \mapsto\left\langle\Phi_{\gamma}\right\rangle_{t}$ is real analytic, $\gamma \mapsto\left\langle\Phi_{\gamma}\right\rangle_{+}$is entire analytic, but the limit and derivative in the expression $\left.\partial_{\gamma}\left(\lim _{t \rightarrow \infty}\left\langle\Phi_{\gamma}\right\rangle_{t}\right)\right|_{\gamma=0}$ cannot be interchanged.

\section{Gaussian dynamical systems}

This class of dynamical systems is treated in detail in the forthcoming article [JLTP] and for reason of space we shall be brief (in particular we will omit all the proofs). The thermally driven harmonic chain of Section 5.5.2 is an example of a Gaussian dynamical system.

Let $\Gamma$ be a countably infinite set and

$$
M=\mathbb{R}^{\Gamma}=\left\{x=\left(x_{n}\right)_{n \in \Gamma} \mid x_{n} \in \mathbb{R}\right\} .
$$

A sequence $l=\left\{l_{n}\right\}_{n \in \Gamma}$ of strictly positive real numbers such that $\sum_{n \in \Gamma} l_{n}=1$ defines a metric

$$
d(x, y)=\sum_{n \in \Gamma} l_{n} \frac{\left|x_{n}-y_{n}\right|}{1+\left|x_{n}-y_{n}\right|},
$$

on $M$. Equipped with $d, M$ is a complete separable metric space. Its Borel $\sigma$-algebra $\mathcal{F}$ is generated by the cylinders

$$
C\left(B ; n_{1}, \ldots, n_{k}\right)=\left\{x \in M \mid\left(x_{n_{1}}, \ldots, x_{n_{k}}\right) \in B\right\},
$$


for $k \geq 0, n_{1}, \ldots, n_{k} \in \Gamma$ and Borel sets $B \subset \mathbb{R}^{k}$.

We denote by $\ell_{\mathbb{R}}^{2}(\Gamma) \subset M$ (respectively $\left.M_{l} \subset M\right)$ the real Hilbert space with the inner product $(x, y)=$ $\sum_{n \in \Gamma} x_{n} y_{n}$ (respectively $\left.(x, y)_{l}=\sum_{n \in \Gamma} l_{n} x_{n} y_{n}\right) . \ell_{\mathbb{R}}^{2}(\Gamma)$ is dense in $M_{l}$ and $M_{l}$ is dense in $M$. All the measures on $(M, \mathcal{F})$ we will consider in this example will be supported on $M_{l}$. We denote by $A_{n m}=\left(\delta_{n}, A \delta_{m}\right)$ the matrix elements of a linear operator $A$ on $\ell_{\mathbb{R}}^{2}(\Gamma)$ w.r.t. its standard basis $\left\{\delta_{n}\right\}_{n \in \Gamma}$.

Let $\mathcal{L}$ be a bounded linear operator on $\ell_{\mathbb{R}}^{2}(\Gamma)$ which has a continuous extension to $M_{l}$. For $x \in M$ and $t \in \mathbb{R}$ we set

$$
\phi^{t}(x)= \begin{cases}\mathrm{e}^{t \mathcal{L}_{x}} & \text { if } x \in M_{l}, \\ x & \text { if } x \notin M_{l} .\end{cases}
$$

$\phi^{t}$ is a group of automorphisms of $(M, \mathcal{F})$ describing the time evolution. Note that the map $(t, x) \mapsto \phi^{t}(x)$ is measurable and so Assumptions (F1) (F2) of Section 2.3 hold for $(M, \phi)$.

Let $D$ be a bounded, strictly positive operator on $\ell_{\mathbb{R}}^{2}(\Gamma)$. The centered Gaussian measure on $(M, \mathcal{F})$ of covariance $D$ is the unique measure $\omega$ specified by its value on cylinders

$$
\omega\left(C\left(B ; n_{1}, \ldots, n_{k}\right)\right)=\frac{1}{\sqrt{(2 \pi)^{k} \operatorname{det} D_{c}}} \int_{B} \mathrm{e}^{-\frac{1}{2}\left(x, D_{c}^{-1} x\right)} \mathrm{d} x,
$$

where $D_{c}=\left[D_{n_{i} n_{j}}\right]_{1 \leq i, j \leq k}$. For any finite subset $\Lambda \subset \Gamma$ one has

$$
\int_{M} \sum_{n \in \Lambda} l_{n} x_{n}^{2} \mathrm{~d} \omega(x)=\sum_{n \in \Lambda} l_{n} D_{n n} \leq\|D\|
$$

which shows that $\omega\left(M_{l}\right)=1$.

Our starting point is the dynamical system $(M, \phi, \omega) \cdot \omega_{t}=\omega \circ \phi^{-t}$ is a Gaussian measure of covariance

$$
D_{t}=\mathrm{e}^{t \mathcal{L}} D \mathrm{e}^{t \mathcal{L}^{*}} .
$$

$D_{t}$ is a bounded strictly positive operator on $\ell_{\mathbb{R}}^{2}(\Gamma)$ and $\omega_{t}\left(M_{l}\right)=1$ for all $t$. Denote by $\mathcal{T}$ the real vector space of all trace class operators on $\ell_{\mathbb{R}}^{2}(\Gamma)$. The trace norm $\|T\|_{1}=\operatorname{tr}\left(\left(T^{*} T\right)^{1 / 2}\right)$ turns $\mathcal{T}$ into a Banach space. By the Feldman-Hajek-Shale theorem $\omega_{t}$ and $\omega$ are equivalent iff $D_{t}^{-1}-D^{-1} \in \mathcal{T}$. We shall assume more:

(G1) The map $\mathbb{R} \ni t \mapsto D_{t}^{-1}-D^{-1} \in \mathcal{T}$ is differentiable.

It follows that

is trace class. Set

$$
\varsigma=-\left.\frac{1}{2} \frac{\mathrm{d}}{\mathrm{d} t}\left(D_{t}^{-1}-D^{-1}\right)\right|_{t=0}=\frac{1}{2}\left(\mathcal{L}^{*} D^{-1}+D^{-1} \mathcal{L}\right),
$$

$$
\sigma(x)=(x, \varsigma x)-\operatorname{tr}(D \varsigma) .
$$

Proposition 9.1 Suppose that (G1) holds. Then:

(1) $\sigma \in L^{1}(M, \mathrm{~d} \omega)$ and $t \mapsto \sigma_{t}$ is strongly continuous in $L^{1}(M, \mathrm{~d} \omega)$.

(2) $\ell_{\omega_{t} \mid \omega}=\int_{0}^{t} \sigma_{-s} \mathrm{~d} s$ and $t \mapsto \mathrm{e}^{\ell_{\omega_{t} \mid \omega}}$ is strongly $C^{1}$ in $L^{1}(M, \mathrm{~d} \omega)$.

(3) $\omega_{t}(\sigma)=\operatorname{tr}\left(\varsigma\left(D_{t}-D\right)\right)$ and in particular $\omega(\sigma)=0$.

(4) $\operatorname{Ent}\left(\omega_{t} \mid \omega\right)=-\int_{0}^{t} \operatorname{tr}\left(\varsigma\left(D_{s}-D\right)\right) \mathrm{d} s$. 
This proposition implies that Assumption (E1) holds and that $\sigma$ is the entropy production observable of $(M, \phi, \omega)$. In some examples only finitely many matrix elements $\varsigma_{n m}$ are non-zero and in this case $\sigma$ is continuous. $\sigma$ is bounded only in the trivial case $\varsigma=0$ and so Assumption[(E2) is not satisfied.

Our next assumptions are:

(G2) For some constants $m_{ \pm}$and all $t \in \mathbb{R}, 0<m_{-} \leq D_{t} \leq m_{+}<\infty$.

(G3) The strong limit

$$
\mathrm{s}-\lim _{t \rightarrow \infty} D_{t}=D_{+}
$$

exists.

Clearly, $m_{-} \leq D_{+} \leq m_{+}$. Let $\omega_{+}$be the Gaussian measure on $(M, \mathcal{F})$ with covariance $D_{+}$.

Proposition 9.2 Suppose that (G1)-(G3) hold. Then:

(1) For all $f \in C_{\mathbb{R}}(M)$,

$$
\lim _{t \rightarrow \infty} \omega_{t}(f)=\omega_{+}(f)
$$

(2) $\sigma \in L^{1}\left(M, \mathrm{~d} \omega_{+}\right)$and

$$
\langle\sigma\rangle_{+}=\lim _{t \rightarrow \infty} \omega_{t}(\sigma)=\operatorname{tr}\left(\varsigma\left(D_{+}-D\right)\right)=\omega_{+}(\sigma)
$$

We shall call $\omega_{+}$the NESS of $(M, \phi, \omega)$. Note that assumptions (NESS2) and (NESS3) of Section6.1 do not hold for $(M, \phi, \omega)$.

Finally, we assume the existence of time reversal in the following form:

(G4) The exists a linear involution $\vartheta$ on $\ell_{\mathbb{R}}^{2}(\Gamma)$ such that $\vartheta \mathcal{L}=-\mathcal{L} \vartheta$ and $\vartheta D=D \vartheta$.

This assumption implies that $D_{-t}=\vartheta D_{t} \vartheta$ also satisfies (G2) and (G3) and

$$
D_{-}=\underset{t \rightarrow-\infty}{\mathrm{s}-\lim _{t \rightarrow}} D_{t}=\vartheta D_{+} \vartheta
$$

Moreover, $\vartheta_{\varsigma}=-\varsigma \vartheta$ and $\operatorname{tr}(D \varsigma)=0$.

Since $\sigma$ is unbounded,

$$
e_{t}(\alpha)=\log \omega\left(\mathrm{e}^{\alpha \int_{0}^{t} \sigma_{-s} \mathrm{~d} s}\right),
$$

is a priori finite only for $\alpha \in[0,1]$. Note that $e_{t}(\alpha)$ is real analytic on $] 0,1[$. (G4) implies that

$$
e_{t}(\alpha)=\log \omega\left(\mathrm{e}^{-\alpha \int_{0}^{t} \sigma_{s} \mathrm{~d} s}\right),
$$

and that $e_{t}(\alpha)=e_{t}(1-\alpha)$. Set $\delta=m_{-} /\left(m_{+}-m_{-}\right)$.

Proposition 9.3 Suppose that (G1)-(G4) hold. Then:

1. $e_{t}(\alpha)$ is finite and real analytic on the interval $]-\delta, 1+\delta[$.

2. $D_{\alpha}=\left((1-\alpha) D_{+}^{-1}+\alpha D_{-}^{-1}\right)^{-1}$ is a real analytic, bounded operator valued function of $\alpha$ on this interval. 
With these preliminaries, the Evans-Searles Fluctuation Theorem holds in the following form.

Theorem 9.4 Suppose that (G1)-(G4) hold. Then:

(1) For $\alpha \in]-\delta, 1+\delta[$,

$$
e(\alpha)=\lim _{t \rightarrow \infty} \frac{1}{t} e_{t}(\alpha)=-\int_{0}^{\alpha} \operatorname{tr}\left(\varsigma D_{\gamma}\right) \mathrm{d} \gamma .
$$

The ES-functional $e(\alpha)$ is real analytic and convex on the interval $]-\delta, 1+\delta[$, satisfies the ES-symmetry and $e^{\prime}(0)=-\langle\sigma\rangle_{+}$.

(2) If $t_{n}$ is a regular sequence, then

$$
\lim _{n \rightarrow \infty} \frac{1}{t_{n}} \int_{0}^{t_{n}} \sigma_{s}(x) \mathrm{d} s=\langle\sigma\rangle_{+}
$$

for $\omega$-a.e. $x$.

(3) The Large Deviation Principle holds in the following form. The function

$$
I(s)=\inf _{\alpha \in]-\delta, 1+\delta[}(\alpha s+e(\alpha)) .
$$

is a concave with values in $[-\infty, 0], I(s)=0$ iff $s=\langle\sigma\rangle_{+}$and $I(s)=s+I(-s)$. Moreover, there is $\epsilon>0$ such that for any open interval $J \subset]-\langle\sigma\rangle_{+}-\epsilon,\langle\sigma\rangle_{+}+\epsilon[$,

$$
\lim _{t \rightarrow \infty} \frac{1}{t} \log \omega\left(\left\{x \mid \frac{1}{t} \int_{0}^{t} \sigma_{s}(x) \mathrm{d} s \in J\right\}\right)=\sup _{s \in J} I(s) .
$$

We now turn to the Gallavotti-Cohen Fluctuation Theorem. Let

$$
e_{t+}(\alpha)=\log \omega_{+}\left(\mathrm{e}^{-\alpha \int_{0}^{t} \sigma_{s} \mathrm{~d} s}\right)
$$

A priori $e_{t+}(\alpha)$ might not be finite for any $\alpha$.

Proposition 9.5 Suppose that (G1)-(G4) hold. Then:

(1) $e_{t+}(\alpha)$ is real analytic on the interval $]-\delta, 1+\delta[$ and for any $\alpha$ in this interval,

$$
e_{+}(\alpha)=\lim _{t \rightarrow \infty} \frac{1}{t} e_{t+}(\alpha)=-\int_{0}^{\alpha} \operatorname{tr}\left(\varsigma D_{\gamma}\right) \mathrm{d} \gamma=e(\alpha) .
$$

In particular, $\left(M, \phi, \omega, \omega_{+}\right)$has regular entropic fluctuations.

(2) If $t_{n}$ is a regular sequence, then

$$
\lim _{n \rightarrow \infty} \frac{1}{t_{n}} \int_{0}^{t_{n}} \sigma_{s}(x) \mathrm{d} s=\langle\sigma\rangle_{+},
$$

for $\omega_{+}$-a.e. $x$.

(3) The Large Deviation Principle holds for $\sigma$ and $\left(M, \phi, \omega_{+}\right)$, i.e., for some $\epsilon>0$ and for any open interval $J \subset]-\langle\sigma\rangle_{+}-\epsilon,\langle\sigma\rangle_{+}+\epsilon[$,

$$
\lim _{t \rightarrow \infty} \frac{1}{t} \log \omega_{+}\left(\left\{x \mid \frac{1}{t} \int_{0}^{t} \sigma_{s}(x) \mathrm{d} s \in J\right\}\right)=\sup _{s \in J} I(s) .
$$


The proofs of all the results described in this section can be found in [JLTP] and here we will only sketch the computations leading to the Formulas (9.66) and (9.67).

Using

$$
\partial_{\alpha} e_{t}(\alpha)=-\frac{\omega\left(\left[\int_{0}^{t} \sigma_{s} \mathrm{~d} s\right] \mathrm{e}^{-\alpha \int_{0}^{t} \sigma_{s} \mathrm{~d} s}\right)}{\omega\left(\mathrm{e}^{-\alpha \int_{0}^{t} \sigma_{s} \mathrm{~d} s}\right)},
$$

and the fact that $\mathrm{e}^{-\alpha \int_{0}^{t} \sigma_{s}} \mathrm{~d} \omega / \omega\left(\mathrm{e}^{-\alpha \int_{0}^{t} \sigma_{s} \mathrm{~d} s}\right)$ is a Gaussian measure of covariance $\left[(1-\alpha) D^{-1}+\alpha D_{-t}^{-1}\right]^{-1}$, we get

$$
\begin{aligned}
e_{t}(\alpha) & =-\int_{0}^{\alpha} \int_{0}^{t} \operatorname{tr}\left(\mathrm{e}^{s \mathcal{L}^{*}} \varsigma \mathrm{e}^{s \mathcal{L}}\left[(1-\gamma) D^{-1}+\gamma D_{-t}^{-1}\right]^{-1}\right) \mathrm{d} s \mathrm{~d} \gamma \\
& =-\int_{0}^{\alpha} \int_{0}^{t} \operatorname{tr}\left(\varsigma\left[(1-\gamma) D_{s}^{-1}+\gamma D_{-t+s}^{-1}\right]^{-1}\right) \mathrm{d} s \mathrm{~d} \gamma \\
& =-t \int_{0}^{\alpha} \int_{0}^{1} \operatorname{tr}\left(\varsigma\left[(1-\gamma) D_{t s}^{-1}+\gamma D_{-t(1-s)}^{-1}\right]^{-1}\right) \mathrm{d} s \mathrm{~d} \gamma,
\end{aligned}
$$

and so

$$
\lim _{t \rightarrow \infty} \frac{1}{t} e_{t}(\alpha)=-\lim _{t \rightarrow \infty} \int_{0}^{\alpha} \int_{0}^{1} \operatorname{tr}\left(\varsigma\left[(1-\gamma) D_{t s}^{-1}+\gamma D_{-t(1-s)}^{-1}\right]^{-1}\right) \mathrm{d} s \mathrm{~d} \gamma=-\int_{0}^{\alpha} \operatorname{tr}\left(\varsigma D_{\gamma}\right) \mathrm{d} \gamma .
$$

Regarding (9.67), we have

$$
\partial_{\alpha} e_{t+}(\alpha)=-\frac{\omega_{+}\left(\left[\int_{0}^{t} \sigma_{s} \mathrm{~d} s\right] \mathrm{e}^{-\alpha \int_{0}^{t} \sigma_{s} \mathrm{~d} s}\right)}{\omega_{+}\left(\mathrm{e}^{-\alpha \int_{0}^{t} \sigma_{s} \mathrm{~d} s}\right)},
$$

where $\mathrm{e}^{-\alpha \int_{0}^{t} \sigma_{s}} \mathrm{~d} \omega_{+} / \omega\left(\mathrm{e}^{-\alpha \int_{0}^{t} \sigma_{s} \mathrm{~d} s}\right)$ is a Gaussian measure with covariance $\left[D_{+}^{-1}+\alpha D_{-t}^{-1}-\alpha D^{-1}\right]^{-1}$. Proceeding as before, we get

$$
e_{t+}(\alpha)=-t \int_{0}^{\alpha} \int_{0}^{1} \operatorname{tr}\left(\varsigma\left[D_{+}^{-1}+\gamma D_{-t(1-s)}^{-1}-\gamma D_{t s}^{-1}\right]^{-1}\right) \mathrm{d} s \mathrm{~d} \gamma
$$

and hence,

$$
\lim _{t \rightarrow \infty} \frac{1}{t} e_{t+}(\alpha)=-\lim _{t \rightarrow \infty} \int_{0}^{\alpha} \int_{0}^{1} \operatorname{tr}\left(\varsigma\left[D_{+}^{-1}+\gamma D_{-t(1-s)}^{-1}-\gamma D_{t s}^{-1}\right]^{-1}\right) \mathrm{d} s \mathrm{~d} \gamma=-\int_{0}^{\alpha} \operatorname{tr}\left(\varsigma D_{\gamma}\right) \mathrm{d} \gamma .
$$

We finish with several remarks.

Regarding the resonance interpretation of $e(\alpha)$ and $e_{+}(\alpha)$, since $\sigma$ is unbounded the study of Liouvilleans and their resolvents requires some care. Regarding generalized functionals and symmetries, if $\mathcal{L}_{X}$ and $D_{X}$ depend on control parameters $X$, then under mild additional regularity assumptions one can compute $g(X, Y), g_{+}(X, Y)$ and prove the Fluctuation Dissipation Theorem. Again, $g(X, Y)=g_{+}(X, Y)$ and the principle of regular fluctuations holds.

\section{Homeomorphisms of compact metric spaces}

Let $(M, d)$ be a compact metric space and $\phi: M \rightarrow M$ a homeomorphism. In this section we consider the discrete time dynamical system on $M$ generated by $\phi$. We shall show, using the thermodynamic formalism, that 
if $\phi$ is sufficiently "chaotic", then the ES and GC Fluctuation Theorems, the Fluctuation-Dissipation theorem and the principle of regular entropic fluctuations hold for a large class of reference states $\omega$. Our treatment generalizes [MV, Ma1]. Some of the results presented here extend also to flows (see Remark 4 on Anosov flows at the end Section 11.

The material in this section is organized as follows. Subsection 10.1 is a brief review of some basic aspects of topological dynamics (see [Wa1] for a more detailed introduction). Subsection 10.2 deals more specifically with two classes of "chaotic" topological dynamics: expansive homeomorphisms with specification (see [KH]) and Smale spaces (see [Ru1]). The reader familiar with these topics can skip Subsections 10.1[10.2 and proceed directly to Subsection 10.3 where, adopting the point of view of [MV], Ma1], we discuss entropy production and fluctuation theorems for chaotic homeomorphisms. We show in Subsection 10.4 how these results relate with the general approach, based on the notion of reference state, advocated in this work. Finally, we discuss, as an example, the simple case of a topological Markov chain in Subsection 10.5 .

\subsection{Topological dynamics}

\subsubsection{Entropy and pressure}

Let $\nu \in \mathcal{S}_{I}$ and let $\xi=\left(C_{1}, C_{2}, \ldots, C_{r}\right), C_{j} \in \mathcal{F}$, be a finite measurable partition of $M$. By a standard subadditivity argument, the limit

$$
h_{\nu}(\phi, \xi)=-\lim _{k \rightarrow \infty} \frac{1}{k} \sum_{j_{1}, \ldots, j_{k} \in\{1, \ldots, r\}} \nu\left(\phi^{-1}\left(C_{j_{1}}\right) \cap \cdots \cap \phi^{-k}\left(C_{j_{k}}\right)\right) \log \nu\left(\phi^{-1}\left(C_{j_{1}}\right) \cap \cdots \cap \phi^{-k}\left(C_{j_{k}}\right)\right),
$$

exists. The Kolmogorov-Sinai entropy of $\phi$ w.r.t. $\nu \in \mathcal{S}_{I}$ is given by

$$
h_{\nu}(\phi)=\sup _{\xi} h_{\nu}(\phi, \xi) .
$$

The map $\nu \mapsto h_{\nu}(\phi)$ is affine, that is,

$$
h_{\lambda \nu+(1-\lambda) \mu}(\phi)=\lambda h_{\nu}(\phi)+(1-\lambda) h_{\mu}(\phi),
$$

for any $\nu, \mu \in \mathcal{S}_{I}$ and $\lambda \in[0,1]$.

For $x \in M$ and $\epsilon>0$ we denote by

$$
B_{ \pm n}(x, \epsilon)=\left\{y \in M \mid \max _{0 \leq j \leq n-1} d\left(\phi^{ \pm j}(x), \phi^{ \pm j}(y)\right)<\epsilon\right\},
$$

the Bowen ball of order $\pm n$. The following result is known as the Brin-Katok local entropy formula $[\overline{\mathrm{BK}}]$ and is a topological version of the Shannon-McMillan-Breiman theorem.

Theorem 10.1 Suppose that $\nu \in \mathcal{S}_{I}$ is non-atomic and that $h_{\nu}(\phi)$ is finite. Then for $\nu$-a.e. $x \in M$,

$$
\lim _{\epsilon \rightarrow 0} \limsup _{n \rightarrow \infty}-\frac{1}{n} \log \nu\left(B_{n}(x, \epsilon)\right)=\lim _{\epsilon \rightarrow 0} \liminf _{n \rightarrow \infty}-\frac{1}{n} \log \nu\left(B_{n}(x, \epsilon)\right)=h_{\nu}(x) .
$$

The function $h_{\nu}(x)$ is $\phi$-invariant and $\nu\left(h_{\nu}\right)=h_{\nu}(\phi)$. If $\nu$ is ergodic, then $h_{\nu}(x)=h_{\nu}(\phi)$ for $\nu$-a.e. $x$.

For any function $\varphi \in C(M)$ we set

$$
S_{n} \varphi(x)=\sum_{j=0}^{n-1} \varphi \circ \phi^{j}(x)
$$


A set $E \subset M$ is said to be $(n, \epsilon)$-separated if for every $x, y \in E, x \neq y$, we have $y \notin B_{n}(x, \epsilon)$. For $\varphi \in C_{\mathbb{R}}(M)$ let

$$
Z_{n}(\varphi, \epsilon)=\sup _{E} \sum_{x \in E} \mathrm{e}^{S_{n} \varphi(x)}
$$

where the supremum is taken over all $(n, \epsilon)$-separated subsets of $M$. The limit

$$
P(\varphi)=\lim _{\epsilon \rightarrow 0} \limsup _{n \rightarrow \infty} \frac{1}{n} \log Z_{n}(\varphi, \epsilon)
$$

exists and $P(\varphi)$ is called the topological pressure of $\phi$ with respect to the potential $\varphi$. Alternative representations of the pressure are

$$
P(\varphi)=\lim _{\epsilon \rightarrow 0} \limsup _{n \rightarrow \infty} \frac{1}{n} \log \sum_{x \in E_{n, \epsilon}} \mathrm{e}^{S_{n} \varphi(x)}=\lim _{\epsilon \rightarrow 0} \liminf _{n \rightarrow \infty} \frac{1}{n} \log \sum_{x \in E_{n, \epsilon}} \mathrm{e}^{S_{n} \varphi(x)},
$$

where the $E_{n, \epsilon}$ are arbitrary maximal $(n, \epsilon)$-separated sets. The pressure also satisfies the variational principle

$$
P(\varphi)=\sup _{\nu \in \mathcal{S}_{I}}\left(\nu(\varphi)+h_{\nu}(\phi)\right)
$$

The special case $P(0)=\sup _{\nu \in \mathcal{S}_{I}} h_{\nu}(\phi)$ is the topological entropy of $\phi$. An immediate consequence of the variational principle is that $P(\varphi)$ depends only on the topology of $M$ and not on the choice of metric $d$. Another consequence is that the map $C_{\mathbb{R}}(M) \ni \varphi \mapsto P(\varphi)$ is convex and that either $P(\varphi)=+\infty$ for all $\varphi$, or $P(\varphi)$ is finite for all $\varphi$. In what follows we assume that $P(\varphi)$ is finite for all $\varphi$. Occasionally we shall use an additive normalization $\widehat{\varphi}=\varphi-P(\varphi)$ which ensures that $P(\widehat{\varphi})=0$.

\subsubsection{Potentials and equilibrium states}

An invariant measure $\nu$ is called an equilibrium state for the potential $\varphi$ if the supremum in (10.71) is realized at $\nu$. In the case $\varphi=0$ the equilibrium states are called measures of maximal entropy. In general, equilibrium states do not necessarily exist, and if they exist they are not necessarily unique. The set $\mathcal{S}_{\text {eq }}(\varphi)$ of all equilibrium states for $\varphi$ is obviously convex. If $\mathcal{S}_{\text {eq }}(\varphi)$ is singleton, we denote by $\nu_{\varphi}$ the unique equilibrium state for $\varphi$.

Theorem 10.2 Suppose that the entropy map

$$
\mathcal{S}_{I} \ni \nu \mapsto h_{\nu}(\phi)
$$

is upper-semicontinuous. Then:

(1) For all $\varphi \in C_{\mathbb{R}}(M)$ the set $\mathcal{S}_{\mathrm{eq}}(\varphi)$ is non-empty and compact. A measure $\nu$ is an extreme point of $\mathcal{S}_{\mathrm{eq}}(\varphi)$ iff $\nu$ is $\phi$-ergodic.

(2) For a dense set of $\varphi$ in $C_{\mathbb{R}}(M)$ the set $\mathcal{S}_{\mathrm{eq}}(\varphi)$ is a singleton.

(3) For $\varphi \in C_{\mathbb{R}}(M)$, the map $\mathbb{R}^{N} \ni Y \mapsto P(\varphi+Y \cdot \mathbf{f})$ is differentiable at 0 for all $\mathbf{f} \in C_{\mathbb{R}}(M)^{N}$ iff $\mathcal{S}_{\text {eq }}(\varphi)$ is a singleton and in this case

$$
\left.\nabla_{Y} P(\varphi+Y \cdot \mathbf{f})\right|_{Y=0}=\nu_{\varphi}(\mathbf{f}) .
$$


The proofs of (1), (2) and (3) for $N=1$ can be found in [Wa1]. For a proof of (3) for $N>1$ see [Je].

For a given $\varphi \in C_{\mathbb{R}}(M)$ we denote by $\mathcal{S}_{\varphi}$ the collection of all $\nu \in \mathcal{S}$ such that for all $x \in M, n>0$ and sufficiently small $\epsilon>0$,

$$
C_{n}(\epsilon)^{-1} \leq \nu\left(B_{n}(x, \epsilon)\right) \mathrm{e}^{-S_{n} \varphi(x)} \leq C_{n}(\epsilon),
$$

where $C_{n}(\epsilon)>0$ satisfies

$$
\lim _{n \rightarrow \infty} \frac{1}{n} \log C_{n}(\epsilon)=0
$$

The main property of the class $S_{\varphi}$ is the following basic result of Kifer [Ki] which relates it to the large deviation formalism trough the topological pressure.

Proposition 10.3 For $\nu \in \mathcal{S}_{\varphi}$ and $\psi \in C_{\mathbb{R}}(M)$,

$$
\lim _{n \rightarrow \infty} \frac{1}{n} \log \nu\left(\mathrm{e}^{S_{n} \psi}\right)=P(\varphi+\psi) .
$$

Proof. Let $E_{n, \epsilon}$ be arbitrary maximal $(n, \epsilon)$-separated sets. Note that for two different $x, y \in E_{n, \epsilon}$ one has $B_{n}(x, \epsilon / 2) \cap B_{n}(y, \epsilon / 2)=\emptyset$ and by maximality $\cup_{x \in E_{n, \epsilon}} B_{n}(x, \epsilon)=M$. It follows that

$$
\sum_{x \in E_{n, \epsilon}} \nu\left(1_{B_{n}(x, \epsilon / 2)} \mathrm{e}^{S_{n} \psi}\right) \leq \nu\left(\mathrm{e}^{S_{n} \psi}\right) \leq \sum_{x \in E_{n, \epsilon}} \nu\left(1_{B_{n}(x, \epsilon)} \mathrm{e}^{S_{n} \psi}\right),
$$

where $1_{B}$ denotes the indicator function of the set $B$. Setting $\delta_{\epsilon}=\sup _{x, y \in M, d(x, y)<\epsilon}|\psi(x)-\psi(y)|$, we obtain

$$
\sum_{x \in E_{n, \epsilon}} \nu\left(B_{n}(x, \epsilon / 2)\right) \mathrm{e}^{S_{n} \psi(x)-n \delta_{\epsilon}} \leq \nu\left(\mathrm{e}^{S_{n} \psi}\right) \leq \sum_{x \in E_{n, \epsilon}} \nu\left(B_{n}(x, \epsilon)\right) \mathrm{e}^{S_{n} \psi(x)+n \delta_{\epsilon}} .
$$

Combining these estimates with (10.73) we get

$$
C_{n}(\epsilon / 2)^{-1} \sum_{x \in E_{n, \epsilon}} \mathrm{e}^{S_{n}(\varphi+\psi)(x)-n \delta_{\epsilon}} \leq \nu\left(\mathrm{e}^{S_{n} \psi}\right) \leq C_{n}(\epsilon) \sum_{x \in E_{n, \epsilon}} \mathrm{e}^{S_{n}(\varphi+\psi)(x)+n \delta_{\epsilon}},
$$

and so

$$
\begin{aligned}
& \limsup _{n \rightarrow \infty} \frac{1}{n} \log \sum_{x \in E_{n, \epsilon}} \mathrm{e}^{S_{n}(\varphi+\psi)(x)} \geq \limsup _{n \rightarrow \infty} \frac{1}{n} \log \nu\left(\mathrm{e}^{S_{n} \psi}\right)-\delta_{\epsilon}, \\
& \liminf _{n \rightarrow \infty} \frac{1}{n} \log \sum_{x \in E_{n, \epsilon}} \mathrm{e}^{S_{n}(\varphi+\psi)(x)} \leq \liminf _{n \rightarrow \infty} \frac{1}{n} \log \nu\left(\mathrm{e}^{S_{n} \psi}\right)+\delta_{\epsilon} .
\end{aligned}
$$

Since $\delta_{\epsilon} \downarrow 0$ as $\epsilon \downarrow 0$, the statement now follows from Equ. (10.70).

Corollary 10.4 Suppose that the entropy map 10.72) is upper-semicontinuous and that $\mathcal{S}_{\mathrm{eq}}(\varphi)$ is a singleton. Then, for all $\nu \in \mathcal{S}_{\varphi}$,

$$
\lim _{n \rightarrow \infty} \frac{1}{n} S_{n} f(x)=\nu_{\varphi}(f),
$$

for all $f \in C_{\mathbb{R}}(M)$ and $\nu$-a.e. $x \in M$. In particular, $\nu_{\varphi}$ is the unique NESS of the system $(M, \phi, \nu)$. 
Proof. By Proposition 10.3 , the generating function

$$
\lim _{n \rightarrow \infty} \frac{1}{n} \log \nu\left(\mathrm{e}^{-\alpha S_{n} f}\right)=P(\varphi-\alpha f),
$$

exists for all $\alpha \in \mathbb{R}$. By Theorem 10.2 it is differentiable at $\alpha=0$ so the statement follows from the Gärtner-Ellis Theorem (Proposition 5.11(1)).

As an immediate consequence of the Katok-Brin local entropy formula (Theorem 10.1) we have:

Proposition 10.5 Suppose that $\nu \in \mathcal{S}_{I} \cap \mathcal{S}_{\varphi}$ is non-atomic. Then for small enough $\epsilon$ and $\nu$-a.e. $x \in M$,

$$
h_{\nu}(x)=-\lim _{n \rightarrow \infty} \frac{1}{n} \log \nu\left(B_{n}(x, \epsilon)\right)=-\lim _{n \rightarrow \infty} \frac{1}{n} S_{n} \varphi(x),
$$

and in particular $\nu(\varphi)+h_{\nu}(\phi)=0$.

Corollary 10.6 If $\mathcal{S}_{\varphi}$ is non-empty, then $P(\varphi)=0$. Moreover, if $\nu \in \mathcal{S}_{I} \cap \mathcal{S}_{\varphi}$ is non-atomic, then $\nu \in \mathcal{S}_{\text {eq }}(\varphi)$.

Proof. Setting $\psi=0$ in 10.75) we get $P(\varphi)=0$. This fact and Proposition 10.5 imply the second statement.

We shall say that the potentials $\varphi$ and $\psi$ are physically equivalent, denoted $\varphi \sim \psi$, if

$$
\lim _{n \rightarrow \infty} \frac{1}{n} \sup _{x \in M}\left|S_{n} \varphi(x)-S_{n} \psi(x)\right|=0 .
$$

This clearly defines an equivalence relation on $C_{\mathbb{R}}(M)$. The following facts are easy to prove:

Proposition 10.7 (1) If $\varphi_{1} \sim \psi_{1}$ and $\varphi_{2} \sim \psi_{2}$, then $a \varphi_{1}+b \varphi_{2} \sim a \psi_{1}+b \psi_{2}$ for all $a, b \in \mathbb{R}$.

(2) $\varphi \sim \psi$ implies $P(\varphi)=P(\psi)$ and $\nu(\varphi)=\nu(\psi)$ for all $\nu \in \mathcal{S}_{I}$. In particular, $\mathcal{S}_{\mathrm{eq}}(\varphi)=\mathcal{S}_{\mathrm{eq}}(\psi)$.

(3) $\mathcal{S}_{\varphi}=\mathcal{S}_{\psi}$ iff $\varphi \sim \psi$.

(4) Either $\mathcal{S}_{\varphi} \cap \mathcal{S}_{\psi}=\emptyset$ or $\mathcal{S}_{\varphi}=\mathcal{S}_{\psi}$.

(5) Note that $\mathcal{S}_{\varphi}$ depends on the choice of metric $d$. If $\widetilde{d}$ is a metric equivalent to d (i.e., $C^{-1} d \leq \widetilde{d} \leq C d$ ), then $\mathcal{S}_{\varphi, d}=\mathcal{S}_{\varphi, \widetilde{d}}$

\subsection{Chaotic homeomorphisms}

\subsubsection{Expansiveness and specification}

The non-triviality of $\mathcal{S}_{\varphi}$ can be deduced from suitable "chaoticity" assumptions on $\phi$. A homeomorphism $\phi$ is called expansive if

(ES1) There exists $r>0$ such that if $d\left(\phi^{n}(x), \phi^{n}(y)\right) \leq r$ for all $n \in \mathbb{Z}$ then $x=y$.

$r$ is called expansive constant of $\phi$. If $\phi$ is expansive then $h_{\nu}(\phi)=h_{\nu}(\phi, \xi)$ for any measurable partition $\xi$ such that $\operatorname{diam}(\xi)=\sup \{d(x, y) \mid x, y \in C, C \in \xi\} \leq r$ and the entropy map $\mathcal{S}_{I} \ni \nu \mapsto h_{\nu}(\phi)$ is upper-semicontinuous (see Proposition 6.5 in [Ru1]).

$\phi$ is called an expansive homeomorphism with specification if in addition to (ES1) 
(ES2) For each $\delta>0$, there exists an integer $p(\delta)>0$ such that the following holds: if $a<b$ are integers, $I_{1}, \ldots, I_{n}$ finite intervals of $\mathbb{Z}$ contained in $\{a, \ldots, b\}$ with $\operatorname{dist}\left(I_{j}, I_{k}\right)>p(\delta)$ for $j \neq k$, and $x_{1}, \ldots, x_{n} \in M$, then there is $x \in M$ such that $\phi^{b-a+p(\delta)}(x)=x$, and

$$
d\left(\phi^{k}(x), \phi^{k}\left(x_{i}\right)\right)<\delta,
$$

for $k \in I_{i}, i=1, \ldots, n$.

A potential $\varphi$ is called regular if for all sufficiently small $\epsilon>0$ there exists $C_{\epsilon}>0$ such that for all $x \in M$, all $n>0$ and all $y \in B_{n}(x, \epsilon)$,

$$
\left|S_{n} \varphi(x)-S_{n} \varphi(y)\right|<C_{\epsilon} .
$$

If $\varphi$ is regular so is $\widehat{\varphi}=\varphi-P(\varphi)$.

Expansive homeomorphisms with specification were introduced by Bowen in [Bo1] and have been much studied since then. We recall the following classical result of Bowen [Bo1] (see also [KH]).

Theorem 10.8 Suppose that $\phi$ is an expansive homeomorphism with specification and $\varphi$ a regular potential. Then $\mathcal{S}_{\mathrm{eq}}(\varphi)$ is a singleton and $\nu_{\varphi} \in \mathcal{S}_{\widehat{\varphi}}$.

An expansive homeomorphism with specification $\phi$ has a rich set of periodic points which completely determine the equilibrium state $\nu_{\varphi}$ of a regular potential $\varphi$. Indeed,

$$
\nu_{\varphi}(f)=\lim _{n \rightarrow \infty} \frac{1}{Z_{n}(\varphi)} \sum_{x \in \operatorname{Fix}\left(\phi^{n}\right)} \mathrm{e}^{S_{n} \varphi(x)} f(x),
$$

for all $f \in C(M)$, where

$$
Z_{n}(\varphi)=\sum_{x \in \operatorname{Fix}\left(\phi^{n}\right)} \mathrm{e}^{S_{n} \varphi(x)}
$$

and $\operatorname{Fix}\left(\phi^{n}\right)=\left\{x \in M \mid \phi^{n}(x)=x\right\}$, the set of periodic points of $\phi$ of period $n$ (see [Bo1]). Moreover, the pressure of $\varphi$ is given by

$$
P(\varphi)=\lim _{n \rightarrow \infty} \frac{1}{n} \log Z_{n}(\varphi),
$$

(see e.g. [KH], Proposition 20.3.3). These two approximation results lead to the following characterization of physical equivalence.

Proposition 10.9 Suppose that $\phi$ is an expansive homeomorphism with specification and that $\varphi, \psi$ are regular potentials. Then the following statements are equivalent:

(1) $\varphi \sim \psi$.

(2) For all $n$ and all $x \in \operatorname{Fix}\left(\phi^{n}\right), S_{n} \varphi(x)=S_{n} \psi(x)$.

Proof. For $x \in \operatorname{Fix}\left(\phi^{n}\right)$ and $k \in \mathbb{N}$, one has $S_{k n}(\varphi-\psi)(x)=k S_{n}(\varphi-\psi)(x)$ and hence

$$
\left|S_{n}(\varphi-\psi)(x)\right| \leq n \frac{1}{k n} \sup _{x \in M}\left|S_{k n}(\varphi-\psi)(x)\right| .
$$

Letting $k \rightarrow \infty$ shows that (1) $\Rightarrow(2)$. Suppose (2) holds, then Equ. 10.77) implies $P(\varphi)=P(\psi)$ and Equ. (10.76) $\nu_{\varphi}=\nu_{\psi}$. Theorem 10.8 now implies that $\nu_{\varphi} \in \mathcal{S}_{\widehat{\varphi}} \cap \mathcal{S}_{\widehat{\psi}}$ and Part (5) of Proposition 10.7yields $\mathcal{S}_{\widehat{\varphi}}=\mathcal{S}_{\widehat{\psi}}$. By Part (4) of the same proposition we have $\widehat{\varphi} \sim \widehat{\psi}$ and, since $P(\varphi)=P(\psi)$, we conclude that $\varphi \sim \psi$. 


\subsubsection{Smale spaces}

One can say more under a stronger "chaoticity" assumption. $(M, \phi)$ is called Smale space if the following holds:

(S1) For some $\epsilon>0$, there exists a continuous map

$$
[\cdot, \cdot]:\{(x, y) \in M \times M \mid d(x, y)<\epsilon\} \rightarrow M,
$$

such that $[x, x]=x,[[x, y], z]=[x, z],[x,[y, z]]=[x, z]$ and $\phi([x, y])=[\phi(x), \phi(y)]$ whenever both sides of these identities are defined.

(S2) For some $\delta>0,0<\lambda<1$ and all $n \in \mathbb{N}$ one has

$$
\begin{gathered}
d\left(\phi^{n}(y), \phi^{n}(z)\right) \leq \lambda^{n} d(y, z) \quad \text { if } \quad y, z \in \bigcap_{m=1}^{\infty} B_{m}(x, \delta), \\
d\left(\phi^{-n}(y), \phi^{-n}(z)\right) \leq \lambda^{n} d(y, z) \quad \text { if } \quad y, z \in \bigcap_{m=1}^{\infty} B_{-m}(x, \delta) .
\end{gathered}
$$

A Smale space is called regular if there exists $C>0$ such that $d(x,[x, y]) \leq C d(x, y)$. A Smale space is topologically + transitive if there exists $x \in M$ such that the set $\left\{\phi^{n}(x) \mid n \geq 0\right\}$ is dense in $M .(M, \phi)$ is a topologically + transitive iff for any open sets $U, V$ and any $N \geq 0$ there exists $n \geq N$ such that $\phi^{n}(U) \cap V \neq \emptyset$. A Smale space is topologically mixing if, for any open sets $U, V$ there exists $N \geq 0$ such that, for all $n \geq N$, $\phi^{n}(U) \cap V \neq \emptyset$. If $(M, \phi)$ is a topologically + transitive Smale space then $\phi$ is an expansive homeomorphism with specification. Note that topologically mixing $\Rightarrow$ topologically + transitive.

Smale spaces can be studied using powerful tools of symbolic dynamics and are very well understood. We will recall some classical result (see Chapter 7 in [Ru1] and in particular Corollaries 7.10 and 7.12). For $\alpha \in] 0,1[$ we denote by $C_{\mathbb{R} / \mathbb{C}}^{\alpha}(M)$ the real/complex vector space of all Hölder continuous functions with exponent $\alpha$, i.e., all $f \in C_{\mathbb{R} / \mathbb{C}}(M)$ such that, for some $C>0$ and all $x, y \in M,|f(x)-f(y)| \leq C d(x, y)^{\alpha}$. The norm

$$
\|f\|_{\alpha}=\sup _{x \neq y} \frac{|f(x)-f(y)|}{d(x, y)^{\alpha}}+\sup _{x}|f(x)|,
$$

turns $C_{\mathbb{R} / \mathbb{C}}^{\alpha}(M)$ into a real/complex Banach space.

Theorem 10.10 Let $(M, \phi)$ be a topologically + transitive Smale space and let $\alpha \in] 0,1[$ be given .

(1) The map $C_{\mathbb{R}}^{\alpha}(M) \ni \varphi \mapsto P(\varphi)$ is real analytic.

(2) For any $\varphi \in C_{\mathbb{R}}^{\alpha}(M)$, $\mathcal{S}_{\mathrm{eq}}(\varphi)$ is singleton and $\nu_{\varphi} \in \mathcal{S}_{\widehat{\varphi}}$.

(3) If $\varphi, \psi \in C_{\mathbb{R}}^{\alpha}(M)$, then $\nu_{\varphi}=\nu_{\psi}$ iff $\varphi$ and $\psi$ are homologous, i.e., $\varphi=\psi+c+f \circ \phi-f$, where $c=P(\varphi)-P(\psi)$ and $f \in C_{\mathbb{R}}(M)$ is unique up to an additive constant. If $(M, \phi)$ is regular, then $f \in C_{\mathbb{R}}^{\alpha}(M)$.

(4) If $\varphi, \psi \in C_{\mathbb{R}}^{\alpha}(M)$, then $\varphi \sim \psi$ iff $\varphi$ and $\psi$ are homologous and $P(\varphi)=P(\psi)$.

Suppose that $(M, \phi)$ is topologically mixing. Then

(5) If $\varphi, f, g \in C_{\mathbb{R}}^{\alpha}(M)$, then for some $A, B>0$ and all $n \in \mathbb{Z}$,

$$
\left|\nu_{\varphi}\left(g f_{n}\right)-\nu_{\varphi}(g) \nu_{\varphi}(f)\right| \leq A \mathrm{e}^{-B|n|} .
$$


(6) Suppose that $\varphi, f^{(1)}, \cdots, f^{(N)} \in C_{\mathbb{R}}^{\alpha}(M)$. Then the Central Limit Theorem holds for $\mathbf{f}=\left(f^{(1)}, \cdots, f^{(N)}\right)$ w.r.t. $\left(M, \phi, \nu_{\varphi}\right)$ with covariance matrix

$$
D_{j k}=\sum_{n \in \mathbb{Z}}\left[\nu_{\varphi}\left(f^{(j)} f_{n}^{(k)}\right)-\nu_{\varphi}\left(f^{(j)}\right) \nu_{\varphi}\left(f^{(k)}\right)\right] .
$$

Moreover, if $(M, \phi)$ is regular, then $D_{k k}>0$ unless $f^{(k)}$ is homologous to 0.

(7) Let $\varphi, \psi \in C_{\mathbb{R}}^{\alpha}(M)$. Consider the transfer operator

$$
U_{\psi} f=\mathrm{e}^{\psi} f \circ \phi
$$

on $C_{\mathbb{R}}(M)$ and let

$$
\mathcal{R}(z)=\sum_{n=0}^{\infty} \mathrm{e}^{-n z} \nu_{\varphi}\left(U_{\psi}^{n} 1\right), \quad \operatorname{Re} z>\sup _{x}|\psi(x)| .
$$

Then for some $\epsilon>0$ the function $\mathcal{R}(z)$ has a meromorphic continuation to the half-plane

$$
\operatorname{Re} z>P(\psi+\varphi)-P(\varphi)-\epsilon,
$$

and its only singularity is a simple pole at $P(\psi+\varphi)-P(\varphi)$.

(8) Let $\varphi \in C_{\mathbb{R}}^{\alpha}(M)$. Then there exists $\epsilon>0$ and $C_{\epsilon}>0$ such that, for all $\psi \in C_{\mathbb{C}}^{\alpha}(M)$ with $\|\psi\|_{\alpha}<\epsilon$,

$$
\sup _{n>0} \frac{1}{n}\left|\log \nu_{\varphi}\left(\mathrm{e}^{S_{n} \psi}\right)\right| \leq C_{\epsilon} .
$$

For (1)-(6) see [Ru1]. (7) and (8) are implicit in [Ru1, Ba1] and are easily established using the well-known spectral properties of Ruelle transfer operators. Note that (8) and Proposition 5.9 yield CLT for $f^{(1)}, \ldots, f^{(N)} \in C_{\mathbb{R}}^{\alpha}(M)$ w.r.t. $\left(M, \phi, \nu_{\varphi}\right)$.

\subsection{Entropy production}

In this section we suppose that $\phi$ is TRI with a continuous time reversal $\vartheta$. Note that the map $\nu \mapsto \nu \circ \vartheta$ preserves $\mathcal{S}_{I}$. One easily checks, using the definition $\underline{10.68}$, that

$$
h_{\nu \circ \vartheta}(\phi)=h_{\nu}\left(\phi^{-1}\right)=h_{\nu}(\phi) .
$$

It follows that, for any $\varphi \in C_{\mathbb{R}}(M)$,

$$
\begin{aligned}
P(\varphi)=\sup _{\nu \in \mathcal{S}_{I}}\left(\nu(\varphi)+h_{\nu}(\phi)\right) & =\sup _{\nu \in \mathcal{S}_{I}}\left(\nu \circ \vartheta(\varphi)+h_{\nu \circ \vartheta}(\phi)\right) \\
& =\sup _{\nu \in \mathcal{S}_{I}}\left(\nu(\varphi \circ \vartheta)+h_{\nu}(\phi)\right)=P(\varphi \circ \vartheta) .
\end{aligned}
$$

To each potential $\varphi \in C_{\mathbb{R}}(M)$ we associate the function

$$
\widetilde{\sigma}_{\varphi}=\varphi-\varphi \circ \vartheta .
$$

As we shall see in the next section, $\widetilde{\sigma}_{\varphi}$ is closely related to the entropy production observable $\sigma$ of the dynamical system $(M, \phi, \omega)$ (as defined in Equ. (3.15) ) for $\omega \in \mathcal{S}_{\varphi}$. In this section, we investigate the intrinsic properties of $\widetilde{\sigma}_{\varphi}$ and its fluctuations. 
Proposition 10.11 (1) $\widetilde{\sigma}_{\varphi} \circ \vartheta=-\widetilde{\sigma}_{\varphi}$ (compare with Proposition 3.7).

(2) If $\varphi \sim \psi$ (i.e., $\mathcal{S}_{\varphi}=\mathcal{S}_{\psi}$ ), then $\widetilde{\sigma}_{\varphi} \sim \widetilde{\sigma}_{\psi}$.

(3) Suppose that $\mathcal{S}_{\mathrm{eq}}(\varphi)$ is a singleton. Then $\nu_{\varphi}\left(\widetilde{\sigma}_{\varphi}\right)=0$ iff $\nu_{\varphi} \circ \vartheta=\nu_{\varphi}$.

In the remaining statements we assume that $\nu \in \mathcal{S}_{I} \cap \mathcal{S}_{\varphi}$ is non-atomic.

(4) For $\epsilon$ small enough

$$
\lim _{n \rightarrow \infty} \frac{1}{n} \log \frac{\nu\left(B_{n}(x, \epsilon)\right)}{\nu\left(B_{n}\left(\vartheta \circ \phi^{n-1}(x), \epsilon\right)\right)}=\lim _{n \rightarrow \infty} \frac{1}{n} S_{n} \widetilde{\sigma}_{\varphi}(x),
$$

for $\nu$-a.e. $x \in M$. If $\nu$ is ergodic, then

$$
\lim _{n \rightarrow \infty} \frac{1}{n} \log \frac{\nu\left(B_{n}(x, \epsilon)\right)}{\nu\left(B_{n}\left(\vartheta \circ \phi^{n-1}(x), \epsilon\right)\right)}=\nu\left(\widetilde{\sigma}_{\varphi}\right),
$$

for $\nu$-a.e. $x \in M$.

(5) $\nu\left(\widetilde{\sigma}_{\varphi}\right) \geq 0$.

(6) Suppose that $\nu$ is ergodic and that $\nu$ and $\nu \circ \vartheta$ are equivalent measures. Then $\nu\left(\widetilde{\sigma}_{\varphi}\right)=0$.

Remark 1. Apart from (1), all statements of the previous proposition hold with the same proofs if one replaces $\vartheta$ by $\vartheta \circ \phi^{k}$ with an arbitrary $k \in \mathbb{Z}$. Our choice of $k=0$ differs from the one in [MV]. A different choice would not affect any result in this and the next section.

Remark 2. By part (4), the observable $\widetilde{\sigma}_{\varphi}$ associated to a potential $\varphi \in C_{\mathbb{R}}(M)$ quantifies the Brin-Katok local entropy produced by changing the reference point from $x$ to $\vartheta \circ \phi^{n-1}(x)$, i.e., by reversing the orbit of $x$.

Proof. (1)-(2) are obvious.

(3) $\nu_{\varphi}\left(\widetilde{\sigma}_{\varphi}\right)=0$ is equivalent to $\nu_{\varphi}(\varphi)=\nu_{\varphi}(\varphi \circ \vartheta)$ which, by the variational principle and Equ. (10.78), is equivalent to

$$
P(\varphi)=\nu_{\varphi}(\varphi)+h_{\nu_{\varphi}}(\phi)=\nu_{\varphi} \circ \vartheta(\varphi)+h_{\nu_{\varphi} \circ \vartheta}(\phi) .
$$

Hence $\nu_{\varphi} \circ \vartheta$ is also an equilibrium state for $\varphi$ and the uniqueness implies $\nu_{\varphi}=\nu_{\varphi} \circ \vartheta$.

(4) follows easily from the conditions 10.73)-(10.74) and Birkhoff ergodic theorem. If $\nu \in \mathcal{S}_{I} \cap \mathcal{S}_{\varphi}$ is non-atomic, then by Corollary 10.6 $\nu \in \mathcal{S}_{\text {eq }}(\varphi)$. Since $\nu \circ \vartheta \in \mathcal{S}_{I}$, the variational principle and Equ. (10.78) lead to

$$
\nu(\varphi)+h_{\nu}(\phi)=P(\varphi) \geq \nu \circ \vartheta(\varphi)+h_{\nu \circ \vartheta}(\phi)=\nu(\varphi \circ \vartheta)+h_{\nu}(\phi),
$$

and (5) follows.

(6) The Brin-Katok formula and (10.81) imply that $\nu\left(\widetilde{\sigma}_{\varphi}\right)=h_{\nu}(\phi)-h_{\nu}(\phi)=0$.

The fluctuations of the observable $\widetilde{\sigma}_{\varphi}$ in the states $\nu \in \mathcal{S}_{\varphi}$ are described in our next result.

Proposition 10.12 (1) For all $\nu \in \mathcal{S}_{\varphi}$ the functional

$$
\mathbb{R} \ni \alpha \mapsto e_{\varphi}(\alpha)=\lim _{n \rightarrow \infty} \frac{1}{n} \log \nu\left(\mathrm{e}^{-\alpha S_{n} \widetilde{\sigma}_{\varphi}}\right),
$$

exists and is given by $e_{\varphi}(\alpha)=P\left(\varphi-\alpha \widetilde{\sigma}_{\varphi}\right)$. 
(2) The symmetry $e_{\varphi}(\alpha)=e_{\varphi}(1-\alpha)$ holds.

(3) If $\varphi \sim \psi$, then $e_{\varphi}(\alpha)=e_{\psi}(\alpha)$.

(4) Suppose that the entropy map $\mathcal{S}_{I} \ni \nu \mapsto h_{\nu}(\phi)$ is upper-semicontinuous and that $\mathcal{S}_{\mathrm{eq}}\left(\varphi-\alpha \widetilde{\sigma}_{\varphi}\right)$ is singleton for all $\alpha \in \mathbb{R}$ and let $\nu \in \mathcal{S}_{\varphi}$. Then the Large Deviation Principle holds for $\widetilde{\sigma}_{\varphi}$ w.r.t. $(M, \phi, \nu)$ with the concave rate function $I_{\varphi}(s)=\sup _{\alpha \in \mathbb{R}}\left(\alpha s+e_{\varphi}(\alpha)\right)$ which satisfies the relation $I_{\varphi}(s)=s+I_{\varphi}(-s)$.

Proof. (1) follows from Proposition10.3 Writing $\varphi-\alpha \widetilde{\sigma}_{\varphi}=(1-\alpha) \varphi+\alpha \varphi \circ \vartheta$, (2) follows immediately from Equ. (10.79). (3) is a direct consequence of (1) and Proposition 10.7 (4) follows from Theorem 10.2, Proposition 10.3 and the Gärtner-Ellis Theorem (Proposition 5.11)..

One can introduce control parameters in the above framework and discuss the generalized symmetry and linear response theory. Consider a map $\mathbb{R}^{N} \ni X \mapsto \phi_{X}$, where each $\phi_{X}$ is a homeomorphism of $M$ with continuous time reversal $\vartheta_{X}$, and a map $\mathbb{R}^{N} \ni X \mapsto \varphi_{X} \in C_{\mathbb{R}}(M)$. Let $\widetilde{\sigma}_{X}=\widetilde{\sigma}_{\varphi_{X}}$. We shall assume that there exists $\mathbf{\Phi}_{X}=\left(\Phi_{X}^{(1)}, \cdots, \Phi_{X}^{(N)}\right)$, with $\Phi_{X}^{(j)} \in C_{\mathbb{R}}(M)$, such that

$$
\widetilde{\sigma}_{X} \sim X \cdot \mathbf{\Phi}_{X}
$$

and $\boldsymbol{\Phi}_{X} \circ \vartheta_{X} \sim-\boldsymbol{\Phi}_{X}$. Finally, denote by $\mathcal{S}_{I X}$ the set of $\phi_{X}$-invariant states and by $P_{X}$ the pressure functional for the map $\phi_{X}$.

Proposition 10.13 (1) For all $\nu \in \mathcal{S}_{\varphi_{X}}$, the functional

$$
\mathbb{R}^{N} \ni Y \mapsto \mathfrak{g}(X, Y)=\lim _{n \rightarrow \infty} \frac{1}{n} \log \nu\left(\mathrm{e}^{-S_{n}\left(Y \cdot \mathbf{\Phi}_{X}\right)}\right),
$$

exists and is given by $\mathfrak{g}(X, Y)=P_{X}\left(\varphi_{X}-Y \cdot \boldsymbol{\Phi}_{X}\right)$.

(2) The symmetry $\mathfrak{g}(X, Y)=\mathfrak{g}(X, X-Y)$ holds.

In the remaining statements we assume that the entropy maps $\mathcal{S}_{I X} \ni \nu \mapsto h_{\nu}\left(\phi_{X}\right)$ are upper-semicontinuous.

(3) Suppose that $\mathcal{S}_{\mathrm{eq}}\left(\varphi_{X}\right)$ is a singleton and denote $\nu_{X}=\nu_{\varphi_{X}}$. Then for all $f \in C(M)$ and $\nu \in \mathcal{S}_{\varphi_{X}}$,

$$
\lim _{n \rightarrow \infty} \frac{1}{n} S_{n} f(x)=\nu_{X}(f)
$$

for $\nu$-a.e. $x \in M$.

(4) Suppose that $\mathcal{S}_{\mathrm{eq}}\left(\varphi_{X}\right)$ is a singleton for $X$ small enough and that $\mathfrak{g}(X, Y)$ is $C^{1,2}$ in a neighborhood of $(0,0)$. Then the transport coefficients are defined and satisfy the Onsager reciprocity relations.

(5) Suppose that the assumptions of (4) hold and write $\nu=\nu_{0}$ and $\Phi^{(j)}=\Phi_{0}^{(j)}$. Then $\nu\left(\Phi^{(j)}\right)=0$. Suppose in addition that $\nu\left(\Phi^{(k)} \Phi_{n}^{(j)}\right)=O\left(n^{-1}\right)$ for $n \rightarrow \infty$ and that for some $\epsilon>0$,

$$
\sup _{Y \in D_{\epsilon}, n>0} \frac{1}{n}\left|\log \nu\left(\mathrm{e}^{-S_{n}(Y \cdot \boldsymbol{\Phi})}\right)\right|<\infty .
$$

Then the Fluctuation-Dissipation Theorem holds. 
(6) Suppose that $Y \mapsto \mathfrak{g}(X, Y)$ is differentiable for all $Y$ and let $\nu \in \mathcal{S}_{\varphi_{X}}$. Then the Large Deviation Principle holds for $\boldsymbol{\Phi}_{X}$ w.r.t. $\left(M, \phi_{X}, \nu\right)$ with the concave rate function $I_{X}(s)=\sup _{Y \in \mathbb{R}^{N}}(Y \cdot s+\mathfrak{g}(X, Y))$ which satisfies the GGC-symmetry $I_{X}(s)=X \cdot s+I_{X}(-s)$.

The proof of Proposition 10.13(1) is the same as the proof of Proposition 10.12(1). The proof of the remaining statements is the same as the proof of the corresponding statements in Proposition 5.15.

Assuming "chaoticity" one can say more. For example if $\phi_{X}$ is an expansive homeomorphism with specification and $\varphi_{X}$ and $\boldsymbol{\Phi}_{X}$ are regular, then the entropy maps are upper-semicontinuous, $\mathcal{S}_{\text {eq }}\left(\varphi_{X}-Y \cdot \boldsymbol{\Phi}_{X}\right)$ is a singleton for all $Y$ (Theorem 10.8) and the map $Y \mapsto \mathfrak{g}(X, Y)$ is everywhere differentiable (Theorem 10.2). If $\left(M, \phi_{X}\right)$ is a topological + transitive Smale space and $\varphi_{X}, \boldsymbol{\Phi}_{X}$ are Hölder continuous, then the map $Y \mapsto \mathfrak{g}(X, Y)$ is real analytic. In addition, Theorem 10.10 yields:

Proposition 10.14 Suppose that $\left(M, \phi_{X}\right)$ is a topologically mixing Smale space and that $\varphi_{X}, \boldsymbol{\Phi}_{X}$, are Hölder continuous for $X$ in a neighborhood of 0 . Suppose also that $\mathfrak{g}(X, Y)$ is $C^{1,2}$ in a neighborhood of $(0,0)$. Then the Fluctuation-Dissipation Theorem holds.

\subsection{Reference measure and physical equivalence}

In this section we investigate the relation between the observable $\widetilde{\sigma}_{\varphi}$ introduced in the previous section and the entropy production observable $\sigma$ defined by equ. (3.15). Throughout the section we make the following assumptions:

The homeomorphism $\phi$ admit a time reversal $\vartheta$ and $\varphi \in C_{\mathbb{R}}(M)$ is a potential.

The metric $d_{\vartheta}(x, y)=d(\vartheta(x), \vartheta(y))$ is equivalent to $d$, i.e., that there exists a constant $C>0$ such that $C^{-1} d(x, y) \leq d_{\vartheta}(x, y) \leq C d(x, y)$ for all $x, y \in M$. This requirement is a mild regularity assumption on $\vartheta$. It follows that $d$ is also equivalent to $d+d_{\vartheta}$, so that we can as well assume that $\vartheta$ is isometric.

$\omega \in \mathcal{S}_{\varphi}$ is a TRI reference state and the dynamical system $(M, \phi, \omega)$ satisfies Assumption (C) with an entropy production observable $\sigma=\ell_{\omega_{1} \mid \omega} \circ \phi \in C_{\mathbb{R}}(M)$.

The following key proposition relates $\sigma$ to $\widetilde{\sigma}_{\varphi}$.

Proposition 10.15 Under the above assumptions one has $\sigma \sim \widetilde{\sigma}_{\varphi}$.

Proof. Using the elementary identity $B_{n}\left(\vartheta \circ \phi^{n-1}(x), \epsilon\right)=\vartheta \circ \phi^{n-1}\left(B_{n}(x, \epsilon)\right)$ and the fact that $\omega$ is [TRI we can write

$$
\omega\left(B_{n}\left(\vartheta \circ \phi^{n-1}(x), \epsilon\right)\right)=\omega_{-n+1}\left(B_{n}(x, \epsilon)\right)=\omega\left(\mathrm{e}^{-S_{n-1} \sigma} 1_{B_{n}(x, \epsilon)}\right) .
$$

We derive the inequalities

$$
\mathrm{e}^{-S_{n-1} \sigma(x)-(n-1) \delta_{\epsilon}} \omega\left(B_{n}(x, \epsilon)\right) \leq \omega\left(B_{n}\left(\vartheta \circ \phi^{n-1}(x), \epsilon\right)\right) \leq \mathrm{e}^{-S_{n-1} \sigma(x)+(n-1) \delta_{\epsilon}} \omega\left(B_{n}(x, \epsilon)\right),
$$

where $\delta_{\epsilon}=\sup _{x, y \in M, d(x, y)<\epsilon}|\sigma(x)-\sigma(y)|$. With $c=\max _{x \in M}|\sigma(x)|$, we thus obtain, for arbitrary $n \in \mathbb{N}$, $x \in M$ and $\epsilon>0$,

$$
\mathrm{e}^{-n \delta_{\epsilon}-c} \leq \frac{\omega\left(B_{n}\left(\vartheta \circ \phi^{n-1}(x), \epsilon\right)\right)}{\omega\left(B_{n}(x, \epsilon)\right)} \mathrm{e}^{S_{n} \sigma(x)} \leq \mathrm{e}^{n \delta_{\epsilon}+c} .
$$

Since $\omega \in \mathcal{S}_{\varphi}$ and $\left(S_{n} \varphi\right)\left(\vartheta \circ \phi^{n-1}(x)\right)=S_{n}(\varphi \circ \vartheta)(x)$, the estimate (10.73) leads, for small enough $\epsilon>0$, to

$$
C_{n}(\epsilon)^{-1} \leq \omega\left(B_{n}\left(\vartheta \circ \phi^{n-1}(x), \epsilon\right)\right) \mathrm{e}^{-S_{n}(\varphi \circ \vartheta)(x)} \leq C_{n}(\epsilon) .
$$


Using again (10.73), we obtain

$$
C_{n}(\epsilon)^{-2} \leq \frac{\omega\left(B_{n}(x, \epsilon)\right)}{\omega\left(B_{n}\left(\vartheta \circ \phi^{n-1}(x), \epsilon\right)\right)} \mathrm{e}^{-S_{n} \widetilde{\sigma}_{\varphi}(x)} \leq C_{n}(\epsilon)^{2},
$$

which, combined with (10.83), yields

$$
\frac{1}{n}\left|S_{n}\left(\sigma-\widetilde{\sigma}_{\varphi}\right)(x)\right| \leq \delta_{\epsilon}+\frac{c}{n}+\frac{2}{n} \log C_{n}(\epsilon) .
$$

It follows that

$$
\limsup _{n \rightarrow \infty} \frac{1}{n} \sup _{x \in M}\left|S_{n}\left(\sigma-\tilde{\sigma}_{\varphi}\right)(x)\right| \leq \delta_{\epsilon},
$$

and the proof is completed by noticing that $\delta_{\epsilon} \downarrow 0$ as $\epsilon \downarrow 0$.

Corollary 10.16 (1) The ES-functional of the dynamical system $(M, \phi, \omega)$

$$
\mathbb{R} \ni \alpha \mapsto e(\alpha)=\lim _{n \rightarrow \infty} \frac{1}{n} \log \omega\left(\mathrm{e}^{-\alpha S_{n} \sigma}\right),
$$

exists and satisfies the ES-symmetry $e(1-\alpha)=e(\alpha)$.

In the remaining statements, we assume that $\phi$ is expansive with specification and that the potential $\varphi$ is regular.

(2) The ES-functional $(\alpha)$ is everywhere differentiable.

(3) The ES Fluctuation Theorem hold.

(4) The system $(M, \phi, \omega)$ has a unique NESS $\omega_{+}$and for any $f \in C(M)$,

$$
\lim _{n \rightarrow \infty} \frac{1}{n} S_{n} f(x)=\omega_{+}(f)
$$

holds for $\omega$-a.e. $x \in M$.

(5) The GC Fluctuation Theorem hold.

(6) The principle of regular entropic fluctuations hold.

(7) If $\omega_{+}$in non-atomic, the system is entropy producing iff $\omega_{+} \neq \omega_{+} \circ \vartheta$.

Proof. (1) Proposition 10.3 yields the existence and the relation $e(\alpha)=P(\varphi-\alpha \sigma)$. Proposition 10.15 implies $\varphi-\alpha \sigma \sim \varphi-\alpha \widetilde{\sigma}_{\varphi}$ from which Proposition 10.7 (2) allow us to conclude that $e(\alpha)=e_{\varphi}(\alpha)$. Thus, the ESsymmetry follows either from Proposition 10.12 (2) or directly from Proposition 3.4 (2).

Invoking Theorem 10.8, (2)-(6) are direct consequences of Proposition 10.12. In particular $\omega_{+}=\nu_{\varphi} \in \mathcal{S}_{\varphi}$ so that (7) is a direct application of Proposition 10.11(6).

Remark. Suppose that $(M, \phi)$ is a topologically + transitive Smale space and that $\widetilde{\sigma}_{\varphi}$ and $\sigma$ are Hölder continuous. Applying Propositions 10.10, we conclude that the ES-functional $e(\alpha)$ is real analytic. Moreover, $\widetilde{\sigma}_{\varphi}$ and $\sigma$ are homologous, i.e.,

$$
\widetilde{\sigma}_{\varphi}=\sigma+h \circ \phi-h
$$

for some $h \in C_{\mathbb{R}}(M)$ which is unique up to an additive constant and also Hölder continuous if $(M, \phi)$ is regular.

Invoking Proposition 10.13 similar results can be obtained for systems $\left(M, \phi_{X}, \omega_{X}\right)$, depending on control parameters, with TRI reference states $\omega_{X} \in \mathcal{S}_{\varphi_{X}}$. Note that if $\boldsymbol{\Phi}_{X}$ is a continuous flux relation for the corresponding entropy production observable $\sigma_{X}$, then (10.82) holds. We leave the details to the reader. 


\subsection{Markov chains}

We shall illustrate the results of this section on the simple example of a Markov chain with finitely many states. The set of states is $\Omega=\{1, \cdots, l\}$,

$$
M=\Omega^{\mathbb{Z}}=\left\{x=\left(x_{j}\right)_{j \in \mathbb{Z}} \mid x_{j} \in \Omega\right\},
$$

with the usual product topology and $\phi: M \rightarrow M$ is the left shift, $\phi(x)_{j}=x_{j+1}$. $M$ is metrizable and a convenient metric for our purposes is

$$
d(x, y)=\lambda^{k(x, y)},
$$

where $\lambda \in] 0,1\left[\right.$ is fixed and $k(x, y)=\inf \left\{|j| \mid x_{j} \neq y_{j}\right\}$. If $d(x, y)<1$, then $x_{0}=y_{0}$. It follows that $\phi$ is expansive and that any $r \in] 0,1[$ is an expansive constant. Setting

$$
[x, y]=\left(\ldots, y_{-2}, y_{-1}, x_{0}, x_{1}, \ldots\right) .
$$

one easily shows that $(M, \phi)$ is a regular, topologically mixing Smale space.

Any function $f: M \rightarrow \mathbb{R}$ which depends only on finitely many $x_{j}$ 's is Hölder continuous. The map $\vartheta(x)_{j}=x_{-j}$ is an isometric time-reversal.

Let $\mathbb{P}=\left[p_{i j}\right]_{i, j \in \Omega}$ with $p_{i j}>0, \sum_{j} p_{i j}=1$, be a transition matrix. By the Perron-Frobenius theorem there is a unique probability vector $\bar{p}=\left[\bar{p}_{i}\right]_{i \in \Omega}$ with $\bar{p}_{i}>0$ and $\sum_{i} \bar{p}_{i}=1$ such that $\bar{p} \mathbb{P}=\bar{p}$.

The (two-sided) Markov chain with transition matrix $\mathbb{P}$ is the invariant Borel probability measure $\nu \in \mathcal{S}_{I}$ such that, for any cylinder,

$$
C=\left\{x \in M \mid x_{k}=j_{1}, x_{k+1}=j_{2}, \ldots, x_{k+n-1}=j_{n}\right\},
$$

we have

$$
\nu(C)=\bar{p}_{j_{1}} p_{j_{1} j_{2}} \cdots p_{j_{n-1} j_{n}} .
$$

The assumption that $p_{i j}>0$ implies that $\nu$ is mixing w.r.t. $\phi$.

For the potential

$$
\varphi(x)=\log p_{x_{0} x_{1}},
$$

and the corresponding observable

$$
\tilde{\sigma}_{\varphi}(x)=\varphi(x)-\varphi \circ \vartheta(x)=\log \frac{p_{x_{0} x_{1}}}{p_{x_{0} x_{-1}}} .
$$

one computes

$$
\nu(\varphi)=\sum_{i, j \in \Omega} \bar{p}_{i} p_{i j} \log p_{i j}, \quad \nu\left(\widetilde{\sigma}_{\varphi}\right)=\sum_{i, j \in \Omega} \bar{p}_{i} p_{i j} \log \frac{p_{i j}}{p_{j i}} .
$$

Since $B_{n}\left(x, \lambda^{k}\right)=\left\{y \in M \mid y_{i}=x_{i}\right.$ for $\left.-k \leq i \leq k+n-1\right\}$ for $k \geq 0$, it easily follows that for any $0<\epsilon<1$ there is a constant $C_{\epsilon}$ such that, for all $n>0$,

$$
C_{\epsilon}^{-1} \leq \nu\left(B_{n}(x, \epsilon)\right) \mathrm{e}^{-S_{n} \varphi(x)} \leq C_{\epsilon}
$$

We conclude that $\nu \in \mathcal{S}_{\varphi}$.

The partition $\xi=\left(\left\{x \in M \mid x_{0}=i\right\}\right)_{i \in \Omega}$ has $\operatorname{diam}(\xi)=\lambda<1$. Hence, the Kolmogorov-Sinai entropy can be computed from $h_{\nu}(\phi)=h_{\nu}(\phi, \xi)$ and a simple calculation leads to

$$
h_{\nu}(\phi)=-\sum_{i, j \in \Omega} \bar{p}_{i} p_{i j} \log p_{i j}
$$


The pressure $e_{\varphi}(\alpha)=P\left(\varphi-\alpha \widetilde{\sigma}_{\varphi}\right)$, a real analytic function of $\alpha$, is most easily computed from Equ. 10.77), where we have

$$
Z_{n}\left(\varphi-\alpha \widetilde{\sigma}_{\varphi}\right)=Z_{n}((1-\alpha) \varphi+\alpha \varphi \circ \vartheta)=\sum_{x \in \Omega^{n}}\left(p_{x_{1} x_{2}}^{1-\alpha} p_{x_{2} x_{1}}^{\alpha}\right) \cdots\left(p_{x_{n} x_{1}}^{1-\alpha} p_{x_{1} x_{n}}^{\alpha}\right)=\operatorname{tr} \mathbb{P}_{\alpha}^{n},
$$

with the matrix $\mathbb{P}_{\alpha}=\left[p_{i j}(\alpha)\right], p_{i j}(\alpha)=p_{i j}^{1-\alpha} p_{j i}^{\alpha}$. Since $p_{i j}(\alpha)>0$, the Perron-Frobenius theorem applies to $\mathbb{P}_{\alpha}$ and consequently $e_{\varphi}(\alpha)$ is equal to the logarithm of its dominant eigenvalue. In particular one checks

$$
P(\varphi)=0=\nu(\varphi)+h_{\nu}(\phi)
$$

so that $\nu$ is the unique equilibrium state for the potential $\varphi$. Note that $\mathbb{P}_{\alpha}^{*}=\mathbb{P}_{1-\alpha}$. The resulting identity $\operatorname{tr} \mathbb{P}_{\alpha}^{n}=$ $\operatorname{tr} \mathbb{P}_{1-\alpha}^{n}$ provides an alternative proof of the symmetry $e_{\varphi}(\alpha)=e_{\varphi}(1-\alpha)$.

Proposition 10.11 implies that $\nu\left(\widetilde{\sigma}_{\varphi}\right) \geq 0$ and $\nu\left(\widetilde{\sigma}_{\varphi}\right)=0$ iff $\nu \circ \vartheta=\nu$. The latter condition is easily seen to equivalent to $\bar{p}_{i} p_{i j}=\bar{p}_{j} p_{j i}$ for all $i, j \in \Omega$. In other words, $\nu\left(\widetilde{\sigma}_{\varphi}\right)=0$ iff the Markov chain satisfies detailed balance. Note that in this case $\widetilde{\sigma}_{\varphi}=g \circ \phi-g \sim 0$, with $g(x)=\log \bar{p}_{x_{0}} p_{x_{0} x_{-1}}$.

Let $q=\left[q_{i}\right]_{i \in \Omega}$ be a probability vector and $\omega$ the state uniquely determined by

$$
\omega\left(\left\{x \in M \mid x_{k}=j_{k}, k=-m, \ldots, n\right\}\right)=q_{j_{0}}\left(p_{j_{0} j_{1}} \cdots p_{j_{n-1} j_{n}}\right)\left(p_{j_{0} j_{-1}} \cdots p_{j_{-m+1} j_{-m}}\right) .
$$

A simple calculation shows that $\omega$ is TRI. Moreover, $\omega \in \mathcal{S}_{\varphi}$ provided $q_{i}>0$ for all $i \in \Omega$. Thus, Corollary 10.16 applies to the TRI system $(M, \phi, \omega)$. Note in particular that its entropy production observable

$$
\sigma(x)=\log \frac{q_{x_{0}} p_{x_{0} x_{1}}}{q_{x_{1}} p_{x_{1} x_{0}}}
$$

is homologous to $\widetilde{\sigma}_{\varphi}$. Explicitly, $\widetilde{\sigma}_{\varphi}-\sigma=h \circ \phi-h$ with $h(x)=\log \left(q_{x_{0}} p_{x_{0} x_{-1}}\right)$. The unique NESS of the system is $\omega_{+}=\nu$ and the system is entropy producing iff $\nu$ does not satisfy detailed balance.

Suppose that the transition matrix $\mathbb{P}_{X}=\left[p_{i j}(X)\right]$ depends on the control parameters $X \in \mathbb{R}^{N}$. We assume that the functions $X \mapsto p_{i j}(X)$ are $C^{2}$ and that $p_{i j}(X)>0$ for all $X$. Denote by $\bar{p}(X)=\left[\bar{p}_{i}(X)\right]$ the corresponding equilibrium vector. Let $\varphi_{X}$ be the corresponding potential, $\nu_{X}$ its equilibrium state and set $\widetilde{\sigma}_{X}=\varphi_{X}-\varphi_{X} \circ \vartheta$. We assume that detailed balance holds for $X=0$ so that $\widetilde{\sigma}_{0} \sim 0$.

For each $X \in \mathbb{R}^{N}$ let $q(X)=\left[q_{i}(X)\right]$ be a probability vector such that $q_{i}(X)>0$ and assume that $q(0)=\bar{p}(0)$. Construct the TRI state $\omega_{X}$ as above and denote by $\sigma_{X}$ the corresponding entropy production observable. The detailed balance condition at $X=0$ implies that $\omega_{0}=\nu_{0}$ and hence $\sigma_{0}=0$.

Setting

$$
\mathbf{F}_{X}(i, j)=\int_{0}^{1} \frac{\left(\nabla p_{i j}\right)(u X)}{p_{i j}(u X)} \mathrm{d} u
$$

and $\mathbf{\Phi}_{X}(x)=\mathbf{F}_{X}\left(x_{0}, x_{1}\right)-\mathbf{F}_{X}\left(x_{1}, x_{0}\right)$ we obtain a flux relation,

$$
\sigma_{X} \sim \widetilde{\sigma}_{X} \sim X \cdot \boldsymbol{\Phi}_{X}
$$

such that the map $X \mapsto \boldsymbol{\Phi}_{X} \in C(M)^{N}$ is differentiable and $\boldsymbol{\Phi}_{X} \circ \vartheta \sim-\boldsymbol{\Phi}_{X}$. Arguing as before, the assumption $p_{i j}(X)>0$ and the relation

$$
Z_{n}\left(\varphi_{X}-Y \cdot \boldsymbol{\Phi}_{X}\right)=\operatorname{tr} \mathbb{P}(X, Y)^{n}
$$

where $\mathbb{P}(X, Y)=\left[p_{i j}(X) \mathrm{e}^{-Y \cdot\left(\mathbf{F}_{X}(i, j)-\mathbf{F}_{X}(j, i)\right)}\right]$ imply that $\mathfrak{g}(X, Y)=P_{X}\left(\varphi_{X}-Y \cdot \mathbf{\Phi}_{X}\right)$ is the logarithm of the dominant eigenvalue of $\mathbb{P}(X, Y)$. The perturbation theory of isolated simple eigenvalue further implies that $\mathfrak{g}$ 
is $C^{1,2}$ in a neighborhood of $(0,0)$ and all the conclusions of Propositions 10.13 and 10.14 hold. Finally, for the family $\left(M, \phi_{X}, \omega_{X}, \Phi_{X}\right)$, one shows that

$$
g(X, Y)=g_{+}(X, Y)=\mathfrak{g}(X, Y) .
$$

Remark. The above approach can be used to discuss entropic fluctuations of a wide range of stochastic processes with suitable modifications to accommodate general (non-compact) state space, continuous-time, as well as Gibbs measures rather than Markov measures. The entropic fluctuations of Markov chains were first discussed by Kurchan [Ku1], Lebowitz and Spohn [LS2], and Maes et al. [Ma1, MRV] MN] who used the path measure approach and the Gibbsian formalism.

\section{Anosov diffeomorphisms}

Let $M$ be a compact connected smooth Riemannian manifold with a given Riemannian metric and let $\omega$ be the induced volume measure on $M$. We denote by $\operatorname{Diff}^{k}(M)$ the set of all $C^{k}$ diffeomorphisms of $M$ equipped with the usual $C^{k}$-topology.

$\phi \in \operatorname{Diff}^{1}(M)$ is called Anosov if $M$ is a hyperbolic set, i.e., if there exist constants $0<\lambda<1, K>0$, and a decomposition of the tangent bundle

$$
T M=E^{u} \oplus E^{s},
$$

into $D \phi$-invariant unstable and stable subbundles, such that for each $x \in M$ and every $n \in \mathbb{N}$

$$
\left\|\left.D_{x} \phi^{n}\right|_{E_{x}^{s}}\right\| \leq K \lambda^{n}, \quad\left\|\left.D_{x} \phi^{-n}\right|_{E_{x}^{u}}\right\| \leq K \lambda^{n} .
$$

The above bounds should hold for some norm equivalent to the Riemannian metric of $M$ and there always exists such a norm for which $K=1$.

The set $\mathcal{A}_{k}(M)$ of all $C^{k}$-Anosov diffeomorphisms of $M$ is an open subset of $\operatorname{Diff}^{k}(M)$ (which can be empty). An Anosov diffeomorphism is called transitive if for any two non-empty open sets $U$ and $V$ and any $N \geq 0$ there exists $n>N$ such that $\phi^{-n}(U) \cap V \neq \emptyset$. Any Anosov diffeomorphism on a torus $\mathbb{T}^{n}$ is transitive. More generally, it is conjectured that all Anosov diffeomorphisms are transitive (see $[\mathrm{KH}]$ for various partial results).

In this section we consider dynamical systems $(M, \phi, \omega)$ where $\phi \in \mathcal{A}_{2}(M)$. We use freely notations and results from Section 10

Let $D_{x} \phi: T_{x} M \rightarrow T_{\phi(x)} M$ be the derivative map of $\phi$ at $x$. The entropy production observable of $(M, \phi, \omega)$ is

$$
\sigma(x)=-\log D(x)
$$

where $D(x)=\left|\operatorname{det} D_{x} \phi\right|$ is the Jacobian of $\phi$ at $x$. Setting $D^{u}(x)=\left.\left|\operatorname{det} D_{x} \phi\right|_{E_{x}^{u}}\left|, D^{s}(x)=\right| \operatorname{det} D_{x} \phi\right|_{E_{x}^{s}} \mid$, we shall consider the potential

$$
\varphi(x)=-\log D^{u}(x)
$$

which is known to be Hölder continuous for $\phi \in \mathcal{A}_{2}(M)$ (see [Bo2, [PS1]).

We shall say that the system $(M, \phi, \omega)$ is TRI if it satisfies the conditions of Section 2.5 with a continuous time reversal $\vartheta$.

Remark 1. If $\vartheta \in \operatorname{Diff}^{1}(M)$ then $D_{x} \vartheta$ provides an isomorphism between $E_{x}^{s / u}$ and $E_{\vartheta(x)} E_{\vartheta(x)}^{u / s}$, in particular the stable and unstable subbundles have the same dimension. Moreover,

$$
\log D^{u} \circ \vartheta=-\log D^{s} \circ \phi^{-1}
$$


so that $\sigma$ is homologous to $\widetilde{\sigma}_{\varphi}=\varphi-\varphi \circ \vartheta$,

$$
\widetilde{\sigma}_{\varphi}-\sigma=\log D^{s}-\log D^{s} \circ \phi^{-1} .
$$

We stress however that we shall not assume $\vartheta$ to be of class $C^{1}$ in the following.

Remark 2. One can always construct TRI Anosov systems starting with an Anosov system $(M, \phi, \omega)$ and applying the construction described at the end of Section 2.5. The time reversal obtained in this way is $C^{\infty}$.

The following classical result is known as the Volume Lemma $([\overline{\mathrm{Bo}} 2,[\mathrm{KH}])$ :

Theorem 11.1 If $\phi \in \mathcal{A}_{2}(M)$ then $\omega \in \mathcal{S}_{\varphi}$. More precisely, for sufficiently small $\epsilon>0$ there exists $C_{\epsilon}>0$ such that, for all $x \in M$ and $n>0$,

$$
C_{\epsilon}^{-1} \leq \omega\left(B_{n}(x, \epsilon)\right) \mathrm{e}^{-S_{n} \varphi(x)} \leq C_{\epsilon} .
$$

Note that, by Corollary 10.6 , this implies $P(\varphi)=0$. Set

$$
e_{n}(\alpha)=\omega\left(\mathrm{e}^{-\alpha S_{n} \sigma}\right) \text {. }
$$

If $(M, \phi, \omega)$ is TRI, the finite time ES-theorem (Proposition 3.5) yields that

$$
e_{n}(\alpha)=e_{n}(1-\alpha)
$$

Proposition 10.3 immediately imply the existence of the ES-functional.

Proposition 11.2 Suppose that $\phi \in \mathcal{A}_{2}(M)$. Then for all $\alpha \in \mathbb{R}$,

$$
e(\alpha)=\lim _{n \rightarrow \infty} \frac{1}{n} \log e_{n}(\alpha)=P(\varphi-\alpha \sigma) .
$$

If $(M, \phi, \omega)$ is TRI $\mid$ then

$$
e(\alpha)=e(1-\alpha)
$$

Remark. The symmetry (11.89) is forced by the finite time symmetry (11.88) and so Relation (11.87) (and the fact that $\vartheta$ is $C^{1}$ ) is not used. One gets a direct proof of (11.89) based on (11.87) using the variational principle for the pressure in the same way as in the proof of Proposition 10.12 (2).

To improve Proposition 11.2 we need to assume more. We recall another classical result in the theory of Anosov diffeomorphisms:

Theorem 11.3 Suppose that $\phi \in \mathcal{A}_{2}(M)$ is transitive. Then $(M, \phi)$ is a topologically mixing Smale space.

In particular $\phi$ is expansive with specification and Theorem 10.8 , together with the fact that $P(\varphi)=0$, yield that $\phi$ has a unique equilibrium state $\nu_{\varphi} \in \mathcal{S}_{\varphi}$. Furthermore, by Corollary 10.4

$$
\lim _{n \rightarrow \infty} \frac{1}{n} S_{n} f(x)=\nu_{\varphi}(f),
$$

for all $f \in C(M)$ and $\omega$-a.e. $x$. Thus, $\omega_{+}=\nu_{\varphi}$ is the unique NESS of $(M, \phi, \omega)$. Proposition 10.3 yields that GC-functional exists and is equal to the ES-functional. 
Proposition 11.4 Suppose that $\phi \in \mathcal{A}_{2}(M)$ is transitive. Then for all $\alpha \in \mathbb{R}$,

$$
e_{+}(\alpha)=\lim _{n \rightarrow \infty} \frac{1}{n} \log \omega_{+}\left(\mathrm{e}^{-\alpha S_{n} \sigma}\right)=P(\varphi-\alpha \sigma) .
$$

In particular, $e(\alpha)=e_{+}(\alpha)$ and $\left(M, \phi, \omega, \omega_{+}\right)$has regular entropic fluctuations.

The analyticity of $e(\alpha)$ and $e_{+}(\alpha)$ (Theorem 10.10) yields the respective Large Deviation Principles for the entropy production observable.

Regarding the strict positivity of entropy production we have the following result.

Proposition 11.5 (1) Suppose that $\phi \in \mathcal{A}_{2}(M)$ is transitive. Then $\omega_{+}(\sigma)=0$ iff $\omega_{+} \ll \omega$.

(2) Suppose that all Anosov diffeomorphisms of $M$ are transitive (for example, $M=\mathbb{T}^{n}$ ). Then there is an open dense set $\tilde{\mathcal{A}} \subset \mathcal{A}_{2}(M)$ such that for all $\phi \in \tilde{\mathcal{A}}, \omega_{+}(\sigma)>0$.

Proof. (1) If $\omega_{+} \ll \omega$, then Corollary 5.5 implies that $\omega_{+}(\sigma)=0$. The other direction follows from the result of Ruelle [Ru6]. (2) follows from (1) and the stability result of Sinai [Si] which states that for an open dense set of $\phi$ 's in $\mathcal{A}_{2}(M)$ the NESS is singular w.r.t. $\omega$.

The resonance interpretation of $e_{+}(\alpha)$ follows from Theorem 10.10 (6). The resonances interpretation of $e(\alpha)$ follows from recent results of Baladi and Tsujii [Ba2, Ba3] and Gouezel and Liverani [BKL, GL1, GL2, Li2, LT] on the spectrum of transfer operators in anisotropic Banach spaces and on the zeta function for Anosov maps.

We now turn to the discussion of linear response theory for Anosov diffeomorphisms. Let $k \geq 4$ and let $X \mapsto \phi_{X}$ be a $C^{k}$ map from some neighborhood of the origin in $\mathbb{R}^{N}$ into $\mathcal{A}_{k}(M)$ such that $\omega$ is an invariant state for $\phi=\phi_{0}\left(\mathcal{A}_{k}(M)\right.$ is a Banach manifold so the notion differentiability makes sense). The map $X \mapsto \sigma_{X}=$ $-\log \left|\operatorname{det} D \phi_{X}\right| \in C_{\mathbb{R}}(M)$ is $C^{k-1}$. We shall consider only the flux relation

$$
\boldsymbol{\Phi}_{X}=\left.\int_{0}^{1} \nabla \sigma_{Y}\right|_{Y=u X} \mathrm{~d} u
$$

Clearly, $X \mapsto \Phi_{X}^{(j)} \in C_{\mathbb{R}}(M)$ is $C^{k-2}$.

Theorem 11.6 Suppose that all Anosov diffeomorphisms of $M$ are transitive. Let $k \geq 4$ and let $X \mapsto \phi_{X} \in$ $\mathcal{A}_{k}(M)$ be a $C^{k}$ map from some neighborhood of the origin in $\mathbb{R}^{N}$ such that $\omega$ is an invariant state for $\phi=\phi_{0}$. Suppose that $\left(M, \phi_{X}, \omega\right)$ is TRI with a time-reversal independent of $X$. Then the Fluctuation-Dissipation Theorem holds: the transport coefficients

$$
L_{j k}=\left.\partial_{X_{k}} \omega_{X+}\left(\Phi_{X}^{(j)}\right)\right|_{X=0}
$$

are defined and satisfy the Onsager reciprocity relations

$$
L_{j k}=L_{k j}
$$

For some $A, B>0$ and all $n,\left|\omega\left(\Phi^{(k)} \Phi_{n}^{(j)}\right)\right| \leq A \mathrm{e}^{-B|n|}$ and the Green-Kubo formula

$$
L_{j k}=\frac{1}{2} \sum_{n \in \mathbb{Z}} \omega\left(\Phi^{(k)} \Phi_{n}^{(j)}\right),
$$

holds. The Central Limit Theorem holds for $\boldsymbol{\Phi}$ with covariance matrix $\left[D_{j k}\right]=2\left[L_{j k}\right]$. 
Proof. $\left(M, \phi_{X}\right)$ is a topologically mixing Smale space for $X$ small enough. Arguing as in Propositions 11.2 and 11.4 we deduce that

$$
g(X, Y)=g_{+}(X, Y)=P_{X}\left(\varphi_{X}-Y \cdot \mathbf{\Phi}_{X}\right) .
$$

Ruelle [Ru3] has proven that the map $X \mapsto \varphi_{X} \in C^{\alpha}(M)$ is $C^{k-2}$. Combining this result with Theorem 7 in [KKPW] one deduces that $(X, Y) \mapsto g(X, Y)$ is $C^{1,2}$ in the neighborhood of the origin and the result follows from Proposition 10.14

We finish with some remarks.

Remark 1. The Green-Kubo formula and Onsager reciprocity relations for Anosov diffeomorphisms were first proven in [GR]. This proof was based on explicit computations and the differentiation formula established in [Ru3].

Remark 2. The proof of Theorem 11.6 looks deceptively simple. It stands on the shoulders of deep results established in [Ru3, KKPW].

Remark 3. The linear response theory for Anosov diffeomorphisms can be also established starting with the finite time Green-Kubo formula and following the strategy outlined in Section 5.2. Obviously, the assumptions of the finite time linear response theory discussed in Section 4.3 hold under the conditions of Theorem 11.6 Under the same conditions Ruelle [Ru3] has proven that the functions $X \mapsto \omega_{X+}\left(\Phi_{X}^{(j)}\right)$ are differentiable (see also [KKPW] and [GL2]). That the limit and derivative in the expression (5.41) can be interchanged follows from the results of Gouezel and Liverani [GL2, Li3] (see also [이]).

Remark 4. Most of the results in this section extend to a certain class of Anosov flows for which sufficiently fast mixing has been proved, such as contact Anosov flows and flows with smooth stable and unstable foliations, see [BGM, Ge, Do1, Do2, Li1, BL]. 


\section{Table of Abbreviations and Symbols}

(C)

(E1)

(E2)

(E3)

(F1)

(F2)

(NESS1)

(NESS2)

(NESS3)

(NESS4)

(T1)

(T2)

(T3)

(T4)

ES-functional

GC-functional

GES-functional

GGC-functional

NESS

TRI

$\langle f\rangle_{t}$

$\langle f\rangle_{+}$

$c^{t}$

$\ell_{\nu \mid \omega}$

$\mathbf{L}_{t}=\left[L_{j k t}\right]$

$\mathbf{L}=\left[L_{j k}\right]$

$\mathcal{N}_{\omega}$

$\mathcal{S}_{I}$

$\Delta_{\nu \mid \omega}$

$\Phi_{X}, \Phi_{X}^{(j)}$

$\sigma$

$\Sigma^{t}$

$\Sigma_{X}^{t}$
Equivalence of $\omega$ and $\omega_{t}$

Regularity of $t \mapsto \Delta_{\omega_{t} \mid \omega}$

Boundedness of $\sigma$

Existence of $\langle\sigma\rangle_{+}$

Group property of $\phi^{t}$

Measurability of $(t, x) \mapsto \phi^{t}(x)$

$M$ is a complete separable metric space

Continuity of $(t, x) \mapsto \phi^{t}(x)$

Continuity of $\sigma$

Precompactness of $\left\{t^{-1} \int_{0}^{t} \omega_{s} \mathrm{~d} s \mid t \geq 1\right\}$

$\omega_{0}$ is $\phi_{0}^{t}$-invariant

$\boldsymbol{\Phi}_{X} \circ \vartheta_{X}=-\boldsymbol{\Phi}_{X}$

Differentiability of $\left\langle\boldsymbol{\Phi}_{X}\right\rangle_{t}$ at $X=0$

Existence of $\left\langle\boldsymbol{\Phi}_{X}\right\rangle_{+}$

$\omega\left(\Phi^{(k)} \Phi_{t}^{(j)}\right)=O\left(t^{-1}\right)$

Evans-Searles functional $e(\alpha)$

Gallavoti-Cohen functional $e_{+}(\alpha)$

Generalized Evans-Searles functional $g(X, Y)$

Generalized Gallavoti-Cohen functional $g_{+}(X, Y)$

Non-Equilibrium Steady State(s)

Time-Reversal Invariance

$t^{-1} \int_{0}^{t} \omega\left(f_{s}\right) \mathrm{d} s$

$\lim _{t \rightarrow \infty}\langle f\rangle_{t}$

Entropy cocycle

$\log \Delta_{\nu \mid \omega}$

Finite time Onsager matrix, transport coefficients

Onsager matrix, transport coefficients

Normal states w.r.t. $\omega$

Invariant states

Radon-Nikodym derivative

Flux observables

Entropy production observable

Mean entropy production rate

Mean fluxes page 7

page 12

page 14

page 24

page 7

page 7

page 37

page 37

page 37

page 37

page 18

page 18

page 20

page 26

page 26

page 30

page 38

page 31

page 39

page 37

page 8

page 20

page 24

page 8

page 6

page 20

page 26

page 6

page 7

page 6

page 18

pages 11 and 12

page 9

page 18

\section{References}

[AJPP] Aschbacher, W., Jakšić, V., Pautrat, Y., and Pillet, C.-A.: Transport properties of quasi-free fermions. J. Math. Phys. 48, 032101-1-28 (2007).

[Ba1] Baladi, V.: Positive Transfer Operators and Decay of Correlations. Advanced Series in Nonlinear Dynamics 16. World Scientific, River Edge, NJ (2000).

[Ba2] Baladi, V., and Tsujii, M.: Anisotropic Hölder and Sobolev spaces for hyperbolic diffeomorphisms. Ann. Inst. Fourier, 57, 127-154 (2007). 
[Ba3] Baladi, V., and Tsujii, M.: Dynamical determinants and spectrum for hyperbolic diffeomorphisms. In Probabilistic and Geometric Structures in Dynamics. K. Burns, D. Dolgopyat and Ya. Pesin (editors). Contemp. Math. 469, 29-68 (2008).

[BGM] Bonetto, F., Gentile, G., and Mastropietro, V.: Electric fields on a surface of constant negative curvature. Erg. Th. Dyn. Sys. 20, 681-696 (2000).

[BK] Brin, M., and Katok, A.: On local entropy. Lecture Notes in Mathematics 1007, 30-38. Springer, Berlin, (1983).

[BKL] Blank, M., Keller, G., and Liverani, C.: Ruelle-Perron-Frobenius spectrum for Anosov maps. Nonlinearity 15, 1905-1973 (2002).

[BL] Butterley, O., and Liverani, C.: Smooth Anosov flows: correlation spectra and stability. Journal of Modern Dynamics 1, 301-322 (2007).

[Bo1] Bowen, R.: Some systems with unique equilibrium state. Math. Systems Theory 8, 193-202 (1974).

[Bo2] Bowen, R.: Equilibrium States and the Ergodic Theory of Anosov Diffeomorphisms. Lecture Notes in Mathematics 470. Springer, Berlin (1975).

[BR] Bratteli, O., and Robinson, D. W.: Operator Algebras and Quantum Statistical Mechanics 1. Springer, Berlin (1987).

[Bry] Bryc, W.: A remark on the connection between the large deviation principle and the central limit theorem. Stat. Prob. Lett. 18, 253-256 (1993).

[BS] Beck, C., and Schloögl.: Thermodynamics of chaotic systems. Cambridge Nonlinear Science Series 4. Cambridge University Press, Cambridge (1993).

[CELS1] Chernov, N. I., Eyink, G.L., Lebowitz, J.L., and Sinai, Ya.G.: Derivation of Ohm's law in a deterministic mechanical model. Phys. Rev. Lett. 70, 2209-2212 (1993).

[CELS2] Chernov, N. I., Eyink, G.L., Lebowitz, J.L., and Sinai, Ya.G.: Steady-state electrical conduction in the periodic Lorentz gas. Commun. Math. Phys. 154, 569-601 (1993).

[CG] Cohen, E. G. D., and Gallavotti, G.: Note on two theorems in nonequilibrium statistical mechanics. J. Stat. Phys. 96, 1343-1349 (1999).

[Ch1] Chernov, N. I.: Sinai billiards under small external forces. Ann. Henri Poincaré 2, 197-236 (2001).

[Ch2] Chernov, N. I.: Sinai billiards under small external forces 2. Ann. Henri Poincaré 9, 91-107 (2008).

[CL] Chernov, N. I., and Lebowitz, J. L.: Stationary nonequilibrium states in boundary-driven Hamiltonian systems: Shear flow. J. Stat. Phys. 86, 953-990 (1997).

[CWW] Carberry, D.M., Williams, S.R., Wang, G.M., Sevick, E.M., and Evans J. D.: The Kawasaki identity and the fluctuation Theorem. J. Chem. Phys. 121, 8179-8182 (2004).

[DDM] Dereziński, J., De Roeck, W., and Maes, C.: Fluctuations of quantum currents and unravelings of master equations. J. Stat. Phys. 131, 341-356 (2008).

[DGT] Dorfman, J.R, Gilbert T., Tasaki, S.: An analytical construction of the SRB measures for Baker-type maps. Chaos, 8, 424-442 (1998).

[Do] Dorfman, J.R.: An Introduction to Chaos in Nonequilibrium Statistical Mechanics. Cambridge University Press, Cambridge (1999). 
[Do1] Dolgopyat, D.: Decay of correlations in Anosov flows. Ann. Math. 147, 357-390 (1998).

[Do2] Dolgopyat, D.: Prevalence of rapid mixing in hyperbolic flows. Erg. Th. Dyn. Sys. 18, 1097-1114 (1998).

[DZ] Dembo, A., and Zeitouni, O.: Large Deviations Techniques and Applications. Second edition. Applications of Mathematics, 38. Springer, New York (1998).

[ECM] Evans, D.J., Cohen, E.G.D., and Morriss, G.P.: Probability of second law violation in shearing steady flows. Phys. Rev. Lett. 71, 2401-2404 (1993).

[EH1] Eckmann, J.-P., and Hairer, M.: Non-equilibrium statistical mechanics of strongly anharmonic chains of oscillators. Commun. Math. Phys. 212, 105-164 (2000).

[EH2] Eckmann, J.-P., and Hairer, M.: Spectral properties of hypoelliptic operators. Commun. Math. Phys. 235, 233-253 (2003).

[El] Ellis, R.S.: Entropy, Large Deviations, and Statistical Mechanics. Springer, Berlin (1985). Reprinted in the series Classics of Mathematics (2006).

[EM] Evans, D.J., and Morriss, G.P.: Statistical Mechanics of Nonequilibrium Fluids. Academic Press, New York (1990).

[EPR1] Eckmann, J.-P., Pillet, C.-A., and Rey-Bellet, L.: Non-equilibrium statistical mechanics of anharmonic chains coupled to two heat baths at different temperatures. Commun. Math. Phys. 201, 657-697 (1999).

[EPR2] Eckmann, J.-P., Pillet, C.-A., and Rey-Bellet, L.: Entropy production in nonlinear, thermally driven Hamiltonian systems. J. Stat. Phys. 95, 305-331 (1999).

[ES] Evans, D.J., and Searles, D.J.: Equilibrium microstates which generate second law violating steady states. Phys Rev. E 50, 1645-1648 (1994).

[Ga1] Gallavotti, G.: Chaotic hypothesis: Onsager reciprocity and fluctuation-dissipation theorem. J. Stat. Phys. 84, 899-925 (1996).

[Ga2] Gallavotti, G.: Dynamical ensembles equivalence in fluid mechanics. Physica D 105, 163-184 (1997).

[Ga3] Gallavotti, G.: Chaotic principle: some applications to developed turbulence. J. Stat. Phys. 86, 907-934 (1997).

[GC1] Gallavotti, G., and Cohen, E.G.D.: Dynamical ensembles in nonequilibrium statistical mechanics. Phys. Rev. Lett. 74, 2694-2697 (1995).

[GC2] Gallavotti, G., and Cohen, E. G. D.: Dynamical ensembles in stationary states. J. Stat. Phys. 80, 931-970 (1995).

[Ge] Gentile, G.: Large deviation rule for Anosov flows. Forum Math. 10, 89-118 (1998).

[GL1] Gouezel, S., and Liverani, C.: Banach spaces adapted to Anosov systems Erg. Th. Dyn. Sys. 26, 189-217 (2006).

[GL2] Gouezel, S., and Liverani, C.: Compact locally maximal hyperbolic sets for smooth maps: fine statistical properties. J. Diff. Geom. 79, 433-477 (2008).

[GM] de Groot, S.R., and Mazur, P.: Nonequilibrium Thermodynamics. NorthHolland, Amsterdam (1962). 
[GR] Gallavotti, G., and Ruelle, D.: SRB states and nonequilibrium statistical mechanics close to equilibrium. Commun. Math. Phys. 190, 279-285 (1997).

[Gr1] Green, M.S.: Markoff random processes and the statistical mechanics of time-dependent phenomena. J. Chem. Phys. 20, 1281-1295 (1952).

[Gr2] Green, M.S.: Markoff random processes and the statistical mechanics of time-dependent phenomena. II. Irreversible processes in fluids. J. Chem. Phys. 22, 398-413 (1954).

[GRS] Gallavotti, G., Rondoni, L., and Segre E.: Lyapunov spectra and nonequilibrium ensembles equivalence in 2D fluid mechanics. Physica D 187, 338-357 (2004).

[HH] Hennion, H., and Hervé, L.: Limit Theorems for Markov Chains and Stochastic Properties of Dynamical Systems by Quasi-Compactness. Lecture Notes in Mathematics 1766. Springer, Berlin (2001).

[HHP] Holian, B.L., Hoover, W.G., and Posch, H.A.: Resolution of Loschmidt's paradox: The origin of irreversible behavior in reversible atomistic dynamics. Phys. Rev. Lett. 59, 10-13 (1987).

[Ho] Hoover, E.G.: Molecular Dynamics. Lecture Notes in Physics 258. Springer, Berlin (1986).

[HP] Hille, E., and Phillips, R.S.: Functional Analysis and Semigroups. AMS, Providence, Rhode Island (1957).

[Je] Jenkinson, O.: Rotation, entropy, and equilibrium states. Trans. AMS 353, 3713-3739 (2001).

[JLTP] Jakšić V., Larochelle, V., Tomberg, A., and Pillet, C.-A.: In preparation.

[JP1] Jakšić, V., and Pillet, C.-A.: On entropy production in quantum statistical mechanics. Commun. Math. Phys. 217, 285-293 (2001).

[JP2] Jakšić, V., and Pillet, C.-A.: Mathematical theory of non-equilibrium quantum statistical mechanics. J. Stat. Phys. 108, 787-829 (2002).

[JP3] Jakšić, V., and Pillet, C.-A.: Non-equilibrium steady states of finite quantum systems coupled to thermal reservoirs. Commun. Math. Phys. 226, 131-162 (2002).

[JPP] Jakšić, V., Pautrat, Y., and Pillet, C.-A.: A quantum central limit theorem for sums of IID random variables. J. Math. Phys. 51, 015208 (2010).

[Ka] van Kampen, N.: The case against linear response theory. Physica Norvegica 5, 279-284 (1971).

[KH] Katok, A., and Hasselblatt, B.: Introduction to the Modern Theory of Dynamical Systems. Encyclopedia of mathematics and its applications 54. Cambridge University Press, Cambridge (1995).

[Ki] Kifer, Y.: Large deviations, averaging and periodic orbits of dynamical systems. Commun. Math. Phys. 162, 33-46 (1994).

[KKPW] Katok, A., Knieper, G., Pollicott, M., and Weiss, H.: Differentiability of entropy for Anosov and geodesic flows. Bulletin AMS 22, 285-293 (1990).

[Ko] Korevaar, J.: Tauberian Theory. A Century of Developments. Springer, Berlin, (2004).

[Ku1] Kurchan, J.: Fluctuation theorem for stochastic dynamics. J. Phys. A 31, 3719-3729 (1998).

[Ku2] Kurchan, J.: A quantum fluctuation theorem. Arxiv preprint cond-mat/0007360 (2000). 
[Kub] Kubo, R.: Statistical-mechanical theory of irreversible processes I. General theory and simple applications to magnetic and conduction problems. J. Phys. Soc. Jap. 12, 570-586 (1957).

[KTH] Kubo, R., Toda, M., and Hashitsume, N.: Statistical Physics II. Nonequilibrium Statistical Mechanics. Second edition. Springer Series in Solid-State Sciences 31. Springer, Berlin (1991).

[Li1] Liverani, C.: On contact Anosov flows. Ann. of Math. 159, 1275-1312 (2004).

[Li2] Liverani, C.: Fredholm determinants, Anosov maps and Ruelle resonances. Discrete and Continuous Dynamical Systems 13, 1203-1215 (2005).

[Li3] Liverani, C.: Private communication.

[LS1] Lebowitz, J. L., and Spohn, H.: Stationary non-equilibrium states of infinite harmonic systems. Commun. Math. Phys. 54, 97-120 (1977).

[LS2] Lebowitz, J. L., and Spohn, H.: A Gallavotti-Cohen-type symmetry in the large deviation functional for stochastic dynamics. J. Stat. Phys. 95, 333-365 (1999).

[LT] Liverani, C., and Tsujii, M.: Zeta functions and dynamical systems Nonlinearity 19, 2467-2473 (2006).

[Ma1] Maes, C.: The fluctuation theorem as a Gibbs property. J. Stat. Phys. 95, 367-392 (1999).

[Ma2] Maes, C: On the origin and the use of fluctuation relations for the entropy. Séminaire Poincaré 2, 29-62 (2003).

[MD] Morriss, G.P. and Dettmann, C.P.: Thermostats: Analysis and application. Chaos 8, 321-336 (1998)

[MN] Maes, C., and Netočný, K.: Time-reversal and entropy. J. Stat. Phys. 110, 269-310 (2003).

[MRV] Maes, C., Redig, F., and Verschuere, M.: From global to local fluctuation theorems. Mosc. Math. J. 1, 421-438 (2001).

[MV] Maes, C., and Verbitskiy, E.: Large deviations and a fluctuation symmetry for chaotic homeomorphisms. Commun. Math. Phys. 233, 137-151 (2003).

[On1] Onsager, L.: Reciprocal relations in irreversible processes I. Phys. Rev. 37, 405-426 (1931).

[On2] Onsager, L.: Reciprocal relations in irreversible processes II. Phys. Rev. 38, 2265-2279 (1931).

[OP] Ohya, M. and Petz, D.: Quantum Entropy and its Use. Second edition. Springer, Berlin (2004).

[PH] Posch, H.A., and Hoover, W.G.: Nonequilibrium molecular dynamics of a classical fluid. In Molecular liquids, new perspectives in physics and chemistry, 527-547. Kluwer (1992).

[Pi] Pillet, C.-A.: Entropy production in classical and quantum systems. Markov Proc. Related Fields 7, 145-157 (2001).

[Po] Pollicott, M.: Stability of mixing rates for axiom A attractors. Nonlinearity 16, 567-578 (2003).

[PS1] Pugh, C., and Shub, M.: Stable manifolds and hyperbolic sets. In Proc. Symp. Pure Math. Vol XIV, Berkeley, Calif. 1968, 133-163. Amer. Math. Soc., Providence, 1970.

[Re] Rényi, A.: On measures of information and entropy. In Proc. 4th Berkeley Sympos. Math. Statist. and Prob., Vol. I, 547-561. Univ. California Press, Berkeley, Calif. (1961).

[RM] Rondoni, L., and Mejia-Monasterio, C.: Fluctuations in non-equlibrium statistical mechanics: models, mathematical theory, physical mechanisms. Nonlinearity 20, 1-37 (2007). 
[Ro] de Roeck, W.: Large deviation generating function for currents in the Pauli-Fierz model. Rev. Math. Phys. 21, 549-585 (2009).

[RT1] Rey-Bellet, L., and Thomas, L.E.: Exponential convergence to non-equilibrium stationary states in classical statistical mechanics. Commun. Math. Phys. 225, 305-329 (2002).

[RT2] Rey-Bellet, L., and Thomas, L. E.: Fluctuations of the entropy production in anharmonic chains. Ann. Henri Poincaré 3, 483-502 (2002).

[Ru1] Ruelle, D.: Thermodynamic Formalism. The Mathematical Structure of Equilibrium Statistical Mechanics. Second edition. Cambridge University Press, Cambridge (2004).

[Ru2] Ruelle, D.: Entropy production in nonequilibrium statistical mechanics. Commun. Math. Phys. 189, 365-371 (1997).

[Ru3] Ruelle, D.: Differentiation of SRB states. Commun. Math. Phys. 187, 227-241 (1997). Correction and complements. Commun. Math. Phys. 234, 185-190 (2003).

[Ru4] Ruelle, D.: Smooth dynamics and new theoretical ideas in nonequilibrium statistical mechanics. J. Stat. Phys. 95, 393-468 (1999).

[Ru5] Ruelle, D.: A remark on the equivalence of isokinetic and isoenergetic thermostats in the thermodynamic limit. J. Stat. Phys. 100, 757-763 (2000).

[Ru6] Ruelle, D.: Positivity of entropy production in nonequilibrium statistical mechanics. J. Stat. Phys. 85, $1-23$ (1996).

[RY] Rey-Bellet, L., and Young, L.-S.: Large deviations in non-uniformly hyperbolic dynamical systems. Erg. Th. Dyn. Sys. 28, 587-612 (2008).

[Sh] Shapero, D.: Student project, unpublished.

[Si] Sinai, Y.: Gibbs measures in ergodic theory. Uspehi Mat. Nauk 27, 21-64 (1972). English translation: Russian Math. Surveys 27, 21-69 (1972).

[TG] Tasaki, S., and Gaspard, P.: Fick's law and fractality of nonequilibrium stationary states in a reversible multibaker map. J. Stat. Phys. 81, 935-987 (1995).

[TM] Tasaki, S., and Matsui, T.: Fluctuation theorem, non-equilibrium steady states and Maclennan-Zubarev ensembles of a class of large systems. Fundamental Aspects of Quantum Physics, Tokyo (2001); QP-PQ: Quantum Probab. White Noise Anal., 17, 100 (World Sci., River Edge NJ 2003).

[Wa1] Walters, P.: An Introduction to Ergodic Theory. Graduate Texts in Mathematics 79. Springer, Berlin (1982).

[WL] Wojtkowski, M.P., and Liverani, C.: Conformally symplectic dynamics and symmetry of the Lyapunov spectrum. Commun. Math. Phys. 194, 47-60 (1998).

[Yo] Young, L.-S.: Statistical properties of dynamical systems with some hyperbolicity. Ann. Math. 147, 585-650 (1998). 